\title{
ARITHMETIC STRUCTURES FOR DIFFERENTIAL OPERATORS ON FORMAL SCHEMES
}

\author{
CHRISTINE HUYGHE, TOBIAS SCHMIDT, AND MATTHIAS STRAUCH
}

\begin{abstract}
Let $\mathfrak{o}$ be a complete discrete valuation ring of mixed characteristic $(0, p)$ and $\mathfrak{X}_{0}$ a smooth formal $\mathfrak{o}$-scheme. Let $\mathfrak{X} \rightarrow \mathfrak{X}_{0}$ be an admissible blow-up. In the first part, we introduce sheaves of differential operators $\mathcal{D}_{\mathfrak{X}, k}^{\dagger}$ on $\mathfrak{X}$, for every sufficiently large positive integer $k$, generalizing Berthelot's arithmetic differential operators on the smooth formal scheme $\mathfrak{X}_{0}$. The coherence of these sheaves and several other basic properties are proven. In the second part, we study the projective limit sheaf $\mathcal{D}_{\mathfrak{X}, \infty}=\lim _{k} \mathcal{D}_{\mathfrak{X}, k}^{\dagger}$ and introduce its abelian category of coadmissible modules. The inductive limit of the sheaves $\mathcal{D}_{\mathfrak{X}, \infty}$, over all admissible blow-ups $\mathfrak{X}$, is a sheaf $\mathcal{D}_{\left\langle\mathfrak{X}_{0}\right\rangle}$ on the Zariski-Riemann space of $\mathfrak{X}_{0}$, which gives rise to an abelian category of coadmissible modules. Analogues of Theorems A and $\mathrm{B}$ are shown to hold in each of these settings, i.e., for $\mathcal{D}_{\mathfrak{X}, k}^{\dagger}, \mathcal{D}_{\mathfrak{X}, \infty}$, and $\mathcal{D}_{\left\langle\mathfrak{X}_{0}\right\rangle}$.
\end{abstract}

\section{Contents}

1. Introduction

2. Arithmetic differential operators with congruence level

2.1. The main construction

2.2. First properties

2.3. An invariance theorem for admissible blow-ups

3. Coadmissible $\mathcal{D}$-modules on $\mathfrak{X}$ and the Zariski-Riemann space

3.1. Coadmissible $\mathcal{D}$-modules on $\mathfrak{X}$

3.2. Coadmissible $\mathcal{D}$-modules on the Zariski-Riemann space $\left\langle\mathfrak{X}_{0}\right\rangle$

3.3. Examples

References

\section{INTRODUCTION}

Let $\mathfrak{o}$ be a complete discrete valuation ring of mixed characteristic $(0, p)$, with uniformizer $\varpi$ and fraction field $L$. In [22] some of us (together with D. Patel) have introduced sheaves of arithmetic differential operators $\mathcal{D}_{n, k}^{\dagger}$ on certain semistable formal models $\mathfrak{X}_{n}$ of the rigid analytic projective line over $L$ (for positive integers $k \geqslant n$ ). A key result of [22] is that $\mathfrak{X}_{n}$ is $\mathcal{D}_{n, k}^{\dagger}$-affine. When $n=0$, the formal model $\mathfrak{X}_{0}$ is formally smooth over $\operatorname{Spf}(\mathfrak{o})$, 
and the sheaf $\mathcal{D}_{0,0}^{\dagger}$ equals Berthelot's sheaf of arithmetic differential operators, as defined in [6], and $\mathcal{D}_{0,0}^{\dagger}$-affinity was known before by a result of one of us [20].

In this paper we generalize the construction of 22 and define and study sheaves of arithmetic differential operators on arbitrary admissible formal blow-ups of an arbitrary given smooth formal scheme $\mathfrak{X}_{0}$ over $\mathfrak{o}$.

At the moment, the main application of this generalization is the localization theorem of [17]: in this context $\mathfrak{X}_{0}$ is the smooth model of the flag variety of a connected split reductive group $\mathbb{G}$ over $L$, and the main result of [17] establishes then an anti-equivalence between the category of admissible locally analytic $\mathbb{G}(L)$-representations (with trivial character) [23 and the category of so-called coadmissible equivariant arithmetic $\mathcal{D}$-modules on the system of all formal models of the rigid analytic flag variety of $\mathbb{G}$.

In the following we describe the construction and the main results of this article. Let $\mathfrak{X}_{0}$ be a smooth formal scheme over o and let $\mathcal{D}_{\mathfrak{X}_{0}}^{(m)}$ be Berthelot's sheaf of arithmetic differential operators of level $m$ on $\mathfrak{X}_{0}$ as defined in [6]. For any number $k \geqslant 0$, we have the subalgebra $\mathcal{D}_{\mathfrak{X}_{0}}^{(k, m)}$ consisting of those differential operators which are generated, locally where we have coordinates $x_{1}, \ldots, x_{M}$ and corresponding derivations $\partial_{1}, \ldots, \partial_{M}$, by operators of the form

$$
\varpi^{k|\underline{\nu}|} \underline{\partial}^{\langle\underline{\nu}\rangle_{(m)}}=\varpi^{k\left(\nu_{1}+\ldots+\nu_{M}\right)} \prod_{l=1}^{M} \partial_{l}^{\left\langle\nu_{l}\right\rangle_{(m)}}, \text { where } \partial_{l}^{\left\langle\nu_{l}\right\rangle_{(m)}}=\frac{\left\lfloor\frac{\nu_{l}}{p^{m}}\right\rfloor !}{\nu_{l} !} \partial_{l}^{\nu_{l}} .
$$

Given an admissible blow-up pr : $\mathfrak{X} \rightarrow \mathfrak{X}_{0}$, we let $k_{\mathfrak{X}}$ be the minimal $k$ such that $\varpi^{k} \mathcal{O}_{\mathfrak{X}} \subset \mathcal{I}$ for any coherent ideal sheaf $\mathcal{I}$ on $\mathfrak{X}_{0}$ whose blow-up is $\mathfrak{X}$. Our first basic result, cf. [2.1.12, says that

$$
\mathcal{D}_{\mathfrak{X}}^{(k, m)}:=\operatorname{pr}^{*} \mathcal{D}_{\mathfrak{X}_{0}}^{(k, m)}=\mathcal{O}_{\mathfrak{X}} \otimes_{\mathrm{pr}^{-1} \mathcal{O}_{\mathfrak{X}_{0}}} \operatorname{pr}^{-1} \mathcal{D}_{\mathfrak{X}_{0}}^{(k, m)}
$$

is naturally a sheaf of rings on $\mathfrak{X}$ whenever $k \geqslant k_{\mathfrak{X}}$. We define

$$
\widehat{\mathcal{D}}_{\mathfrak{X}}^{(k, m)}={\underset{\lim }{\longleftrightarrow}}_{i} \mathcal{D}_{\mathfrak{X}}^{(k, m)} / \varpi^{i} \text { and } \mathcal{D}_{\mathfrak{X}, k}^{\dagger}=\underset{m}{\lim } \widehat{\mathcal{D}}_{\mathfrak{X}}^{(k, m)} \otimes \mathbb{Q},
$$

and call these sheaves arithmetic differential operators of congruence leve $k$ on $\mathfrak{X}$.

The structure theory of these differential operators goes largely parallel to the classical smooth setting (when $\mathfrak{X}=\mathfrak{X}_{0}$ and $k=0$ ), as developed by Berthelot [6]. In particular, the sheaves $\mathcal{D}_{\mathfrak{X}}^{(k, m)}, \widehat{\mathcal{D}}_{\mathfrak{X}}^{(k, m)}$ and $\mathcal{D}_{\mathfrak{X}, k}^{\dagger}$ are sheaves of coherent rings on $\mathfrak{X}$. We then show that Cartan's theorems A and B hold for the sheaf $\mathcal{D}_{\mathfrak{X}, k}^{\dagger}$, when restricted to an affine open

\footnotetext{
${ }^{1}$ The terminology is motivated by the relation to congruence subgroups in reductive groups in the case of formal models of flag varieties, cf. [17.
} 
subscheme $\mathfrak{U}$ of $\mathfrak{X}$, cf. 2.2.15, This means that the global sections functor $\Gamma(\mathfrak{U},-)$ furnishes an equivalence of categories between the coherent modules over $\mathcal{D}_{\mathfrak{U}, k}^{\dagger}$ and over $\Gamma\left(\mathfrak{U}, \mathcal{D}_{\mathfrak{X}, k}^{\dagger}\right)$, respectively. A key result (the 'invariance theorem') shows that in case of a morphism $\mathfrak{X}^{\prime} \rightarrow \mathfrak{X}$ between admissible blow-ups of $\mathfrak{X}_{0}$, the categories of coherent modules over $\mathcal{D}_{\mathfrak{X}^{\prime}, k}^{\dagger}$ and over $\mathcal{D}_{\mathfrak{X}, k}^{\dagger}$, respectively, are naturally equivalent, cf. 2.3.8. As a consequence, we obtain global versions of theorem $\mathrm{A}$ and $\mathrm{B}$ on the whole blow-up $\mathfrak{X}$ provided the base $\mathfrak{X}_{0}$ is affine, cf. 2.3.12.

Our next objective is to pass to the projective limit

$$
\mathcal{D}_{\mathfrak{X}, \infty}=\lim _{k} \mathcal{D}_{\mathfrak{X}, k}^{\dagger}
$$

and to define the category $\mathcal{C}_{\mathfrak{X}}$ of coadmissible $\mathcal{D}_{\mathfrak{X}, \infty}$. modules. This is a full abelian sub-

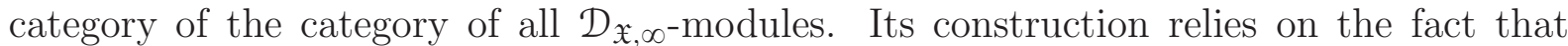
the ring of local sections $\Gamma\left(\mathfrak{V}, \mathcal{D}_{\mathfrak{X}, \infty}\right)$ over an open affine $\mathfrak{V} \subseteq \mathfrak{X}$ is a Fréchet-Stein algebra. Our terminology (as well as the general philosophy behind these constructions) goes back to the fundamental work of P. Schneider and J. Teitelbaum who introduced the concept of a Fréchet-Stein algebra and defined and studied the category of coadmissible modules over such a ring, cf. [23. In fact, we show that the global sections functor $\Gamma(\mathfrak{V},-)$ induces an equivalence of categories between $\mathcal{C}_{\mathfrak{V}}$ and the category of coadmissible $\Gamma\left(\mathfrak{V}, \mathcal{D}_{\mathfrak{X}, \infty}\right)$-modules, cf. [3.1.12. Moreover, any coadmissible $\mathcal{D}_{\mathfrak{X}, \infty}$-module has vanishing higher cohomolgy. These results should be regarded as Cartan's theorems A and B in this setting, cf. 3.1 .12 and 3.1 .15 .

Finally we consider the Zariski-Riemann space of $\mathfrak{X}_{0}$, i.e., the projective $\operatorname{limit}\left\langle\mathfrak{X}_{0}\right\rangle=\lim \mathfrak{X}$ of all admissible formal blow-ups $\mathfrak{X} \rightarrow \mathfrak{X}_{0}$, cf. [8]. One can then form the inductive limit

$$
\mathcal{D}_{\left\langle\mathfrak{X}_{0}\right\rangle}=\underset{\mathfrak{X}}{\lim } \operatorname{sp}_{\mathfrak{X}}^{-1} \mathcal{D}_{\mathfrak{X}, \infty},
$$

where $\operatorname{sp}_{\mathfrak{X}}:\left\langle\mathfrak{X}_{0}\right\rangle \rightarrow \mathfrak{X}$ is the projection map. This is a sheaf of rings on $\left\langle\mathfrak{X}_{0}\right\rangle$ and we define the abelian category of coadmissible $\mathcal{D}_{\left\langle\mathfrak{x}_{0}\right\rangle}$-modules. We establish analogues of Theorems $\mathrm{A}$ and $\mathrm{B}$ in this setting, cf. 3.2.6.

After we developed much of the theory presented here (which began with [21, 22]) we became aware of the article [24] by A. Shiho, where he introduces sheaves of $p$-adic differential operators of negative level $-m$, as they are called there. These are closely related to the sheaves considered here, where the congruence level $k$ corresponds to Shiho's level $-m$. We are currently investigating the implications that Shiho's work has in our context.

We also want to mention that K. Ardakov and S. Wadsley are developing a theory of $D$ modules on general smooth rigid-analytic spaces, cf. [1, 2, 3]. In their work they consider deformations of crystalline differential operators (as in [4]), whereas we take as a starting point deformations of Berthelot's arithmetic differential operators. Though we have not 
carried this out in the present paper, it is not too difficult to see that the category of coadmissible $\mathcal{D}_{\left\langle\mathfrak{x}_{0}\right\rangle}$-modules as defined here, when pulled back to the site of the rigidanalytic space of classical points, coincides with the corresponding category studied in [1, 2, 3].

Acknowledgments. T.S. would like to acknowledge support of the Heisenberg programme of Deutsche Forschungsgemeinschaft (SCHM 3062/1-1). M.S. is grateful for the hospitality and support of the following institutions where work on this project has been accomplished: Institut de Recherche Mathématique Avancée (IRMA) of the University of Strasbourg, Centre Henri Lebesgue, Institut de Recherche Mathématique de Rennes (IRMAR).

Notations and Conventions. We denote by $\varpi$ a uniformizer of the complete discrete valuation ring $\mathfrak{o}$, and we let $|\cdot|_{p}$ be the absolute value on $L=\operatorname{Frac}(\mathfrak{o})$ which is normalized by $|p|_{p}=p^{-1}$. Throughout this paper $\mathfrak{S}=\operatorname{Spf}(\mathfrak{o})$. A formal scheme $\mathfrak{X}$ over $\mathfrak{S}$ such that $\varpi \mathcal{O}_{\mathfrak{X}}$ is an ideal of definition and which is locally noetherian is called a $\mathfrak{S}$-formal scheme. If the $\mathfrak{S}$-formal scheme $\mathfrak{X}$ is smooth over $\mathfrak{S}$ we denote by $\mathcal{T}_{\mathfrak{X}}$ its relative tangent sheaf. A coherent sheaf of ideals $\mathcal{I} \subset \mathcal{O}_{\mathfrak{X}}$ is called open if for any open $\mathfrak{U} \subset \mathfrak{X}$ the restriction of $\mathcal{I}$ to $\mathfrak{U}$ contains $\varpi^{k} \mathcal{O}_{\mathfrak{U}}$ (for some $k \in \mathbb{N}$ depending on $\mathfrak{U}$ ). A scheme which arises from blowing up an open ideal sheaf on $\mathfrak{X}$ will be called an admissible blow-up of $\mathfrak{X}$. For an integer $i \geqslant 0$ we also denote $X_{i}$ the scheme

$$
X_{i}=\mathfrak{X} \times_{\mathfrak{S}} \operatorname{Spec}\left(\mathfrak{o} / \varpi^{i+1} \mathfrak{o}\right)
$$

where the Cartesian product is taken in the category of locally ringed spaces. Without further mentioning, all occuring modules will be left modules. We let $\mathbb{N}=\{0,1,2, \ldots\}$ be the set of non-negative integers.

\section{Arithmetic Differential operators With CONGRUEnCE LEVEL}

Let $\mathfrak{X}_{0}$ be a smooth and separated $\mathfrak{S}$-formal scheme, and let

$$
X_{0, i}=\mathfrak{X}_{0} \times_{\mathfrak{S}} \operatorname{Spec}\left(\mathfrak{o} / \varpi^{i+1} \mathfrak{o}\right) .
$$

Let us write $\mathcal{I}_{\Delta}$ for the diagonal ideal of the closed immersion of formal schemes $\mathfrak{X}_{0} \hookrightarrow$ $\mathfrak{X}_{0} \times \mathfrak{X}_{0}$ and $\mathcal{I}_{\Delta, i}$ for the diagonal ideal : $X_{0, i} \hookrightarrow X_{0, i} \times X_{0, i}$. We also introduce $\mathfrak{X}_{0, \mathbb{Q}}$, the generic fiber of $\mathfrak{X}_{0}$. It is a rigid analytic space over $L$. We write $\overline{\mathcal{I}}_{\Delta}$ for the diagonal ideal of the closed immersion of analytic spaces $\mathfrak{X}_{0, \mathbb{Q}} \hookrightarrow \mathfrak{X}_{0, \mathbb{Q}} \times \mathfrak{X}_{0, \mathbb{Q}}$. We have specialization maps $\mathfrak{X}_{0, \mathbb{Q}} \rightarrow \mathfrak{X}_{0}$ and $\mathfrak{X}_{0, \mathbb{Q}} \times \mathfrak{X}_{0, \mathbb{Q}} \rightarrow \mathfrak{X}_{0} \times \mathfrak{X}_{0}$ which we denote by sp. There is a canonical isomorphism $\operatorname{sp}^{*}\left(\mathcal{I}_{\Delta}\right) \simeq \overline{\mathcal{I}}_{\Delta}$. Finally, the relative tangent sheaf $\mathcal{T}_{\mathfrak{X}_{0}}$ is a locally free $\mathcal{O}_{\mathfrak{X}_{0}}-$ module of finite rank equal to the relative dimension $M$ of $\mathfrak{X}_{0}$ over $\mathfrak{S}$.

\subsection{The main construction.}


2.1.1. Definitions over $\mathfrak{X}_{0}$. The sheaf of relative differential operators of $X_{0, i}$ over $\mathfrak{o} / \varpi^{i+1}$, as introduced in [12, 16.8], will be denoted by $\mathcal{D}_{X_{0, i}}$. It naturally acts on the structure sheaf $\mathcal{O}_{X_{0, i}}$. Its subsheaf $\mathcal{D}_{X_{0, i}}^{n}$ of differential operators of order $\leqslant n$ is defined by

$$
\mathcal{D}_{X_{0, i}}^{n}=\mathcal{H o m}_{\mathcal{O}_{X_{0, i}}}\left(\mathcal{O}_{X_{0, i} \times X_{0, i}} / \mathcal{I}_{\Delta, i}^{n+1}, \mathcal{O}_{X_{0, i}}\right) .
$$

It is a sheaf of locally free $\mathcal{O}_{X_{0, i}}$-modules of finite type and we have $\mathcal{D}_{X_{0, i}}=\lim _{\longrightarrow} \mathcal{D}_{X_{0, i}}^{n}$. For fixed $n$, the projective limit $\lim _{i} \mathcal{D}_{X_{0, i}}^{n}$ is a locally free $\mathcal{O}_{\mathfrak{X}_{0}}$-module of finite type. Taking the inductive limit produces a sheaf of rings

$$
\mathcal{D}_{\mathfrak{X}_{0}}=\underset{n}{\lim _{\longrightarrow}}\left(\varliminf_{i} \mathcal{D}_{X_{0, i}}^{n}\right)
$$

on $\mathfrak{X}_{0}$. It naturally acts on the structure sheaf $\mathcal{O}_{\mathfrak{X}_{0}}$ and can be described in local coordinates as follows. Let $\mathfrak{U}_{0} \subseteq \mathfrak{X}_{0}$ be an open affine endowed with étale coordinates $x_{1}, \ldots, x_{M}$ and corresponding set of derivations $\partial_{1}, \ldots, \partial_{M}$. Write $\partial_{l}^{[\nu]} \in \mathcal{D}_{\mathfrak{L}_{0}}$ for the differential operator defined by $\nu ! \partial_{l}^{[\nu]}=\partial_{l}^{\nu}$, and put $\underline{\nu}=\left(\nu_{1}, \ldots, \nu_{M}\right) \in \mathbb{N}^{M}, \underline{\partial}^{[\underline{\underline{ }}]}=\prod_{l=1}^{M} \partial_{l}^{\left[\nu_{l}\right]}$. One has the following description, involving finite sums,

$$
\mathcal{D}_{\mathfrak{X}_{0}}\left(\mathfrak{U}_{0}\right)=\left\{\sum_{\underline{\underline{\nu}}}^{<\infty} a_{\underline{\underline{\nu}}} \underline{\underline{\underline{\nu}}} \underline{\underline{ }} \mid a_{\underline{\underline{\nu}}} \in \mathcal{O}_{\mathfrak{X}_{0}}\left(\mathfrak{U}_{0}\right)\right\} .
$$

Since $\mathfrak{X}_{0}$ is a smooth $\mathfrak{S}$-formal scheme, one also has the usual sheaves of arithmetic differential operators defined by Berthelot in [6]. In particular, for a fixed non-negative $m, \mathcal{D}_{\mathfrak{X}_{0}}^{(m)}$ will denote the sheaf of differential operators over $\mathfrak{X}_{0}$ of level $m$. Taking $\mathfrak{U}_{0}$ to be endowed with local coordinates $x_{1}, \ldots, x_{M}$ as before, one introduces the following differential operators

$$
\partial_{l}^{\langle\nu\rangle}=q_{\nu} ! \partial_{l}^{[\nu]},
$$

where $q_{\nu}$ denotes the quotient of the euclidean division of $\nu$ by $p^{m}$. For $\underline{\nu}=\left(\nu_{1}, \ldots, \nu_{M}\right) \in$ $\mathbb{N}^{M}$, we also define $\underline{\partial} \underline{\underline{\nu}\rangle}=\prod_{l=1}^{M} \partial_{l}^{\left\langle\nu_{l}\right\rangle}$. Restricted to $\mathfrak{U}_{0}$, the sheaf $\mathcal{D}_{\mathfrak{X}_{0}}^{(m)}$ is a sheaf of free $\mathcal{O}_{\mathfrak{U}_{0}}$-modules with basis given by the elements $\left.\underline{\partial} \underline{\underline{\nu}}\right\rangle$. Berthelot introduces also the following sheaves over $\mathfrak{X}_{0}$

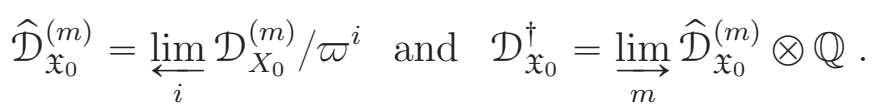

Let $k$ be a non-negative integer, called a congruence level. We define subsheaves $\mathcal{D}_{\mathfrak{X}_{0}}^{(k, m)}$ of subalgebras of the previous sheaves $\mathcal{D}_{\mathfrak{X}_{0}}^{(m)}$ in the following way. Take $\mathfrak{U}_{0}$ endowed with local coordinates $x_{1}, \ldots, x_{M}$ as before. Then the sheaf $\mathcal{D}_{\mathfrak{X}_{0}}^{(k, m)}$ is free over $\mathfrak{U}_{0}$ as a sheaf of 
$\mathcal{O}_{\mathfrak{U}_{0}}$-modules with a basis given by the elements $\varpi^{k \mid \underline{\nu}} \underline{\underline{\partial}} \underline{\underline{\nu}\rangle}$. In particular, one has

$$
\left.\mathcal{D}_{\mathfrak{X}_{0}}^{(k, m)}\left(\mathfrak{U}_{0}\right)=\left\{\sum_{\underline{\nu}}^{<\infty} \varpi^{k \mid \underline{\nu}} a_{\underline{\nu}} \underline{\partial} \underline{\underline{\nu}}\right\rangle \mid a_{\underline{\underline{\nu}}} \in \mathcal{O}_{\mathfrak{X}_{0}}\left(\mathfrak{U}_{0}\right)\right\} .
$$

It is easy to check that these sheaves define a subsheaf of $\mathcal{D}_{\mathfrak{X}_{0}}^{(m)}$ and we call them level $m$ arithmetic differential operators of congruence level $k$ on $\mathfrak{X}_{0}$. We then define as before

$$
\mathcal{D}_{X_{0, i}}^{(k, m)}=\mathcal{D}_{\mathfrak{X}_{0}}^{(k, m)} / \varpi^{i+1}, \widehat{\mathcal{D}}_{\mathfrak{X}_{0}}^{(k, m)}={\underset{\lim }{\longleftarrow}}_{\mathcal{D}_{X_{0, i}}^{(k, m)}} \text { and } \mathcal{D}_{\mathfrak{X}_{0}, k}^{\dagger}=\underset{m}{\lim } \widehat{\mathcal{D}}_{\mathfrak{X}_{0}}^{(k, m)} \otimes \mathbb{Q} \text {. }
$$

Of course, for $k=0$ one recovers the sheaves of Berthelot $\mathcal{D}_{\mathfrak{X}_{0}}^{(0, m)}=\mathcal{D}_{\mathfrak{X}_{0}}^{(m)}$. Note also, by definition, the sheaf $\mathcal{D}_{\mathfrak{X}_{0}, k}^{\dagger}$ is a sheaf of $\mathbb{Q}$-algebras.

We have the following description over an affine open $\mathfrak{U}_{0}$ of $\mathfrak{X}_{0}$ endowed with coordinates $x_{1}, \ldots, x_{M}$

$$
\mathcal{D}_{\mathfrak{X}_{0}, k}^{\dagger}\left(\mathfrak{U}_{0}\right)=\left\{\sum_{\underline{\nu}} \varpi^{k|\underline{\nu}|} a_{\underline{\nu}} \underline{\partial}^{[\underline{\nu}]} \mid a_{\underline{\underline{\nu}}} \in \mathcal{O}_{\mathfrak{X}_{0}, \mathbb{Q}}\left(\mathfrak{U}_{0}\right), \text { and } \exists C>0, \eta<1|| a_{\underline{\nu}} \mid<C \eta^{|\underline{\nu}|}\right\},
$$

where $|\cdot|$ is any Banach norm on the affinoid algebra $\mathcal{O}_{\mathfrak{X}_{0}, \mathbb{Q}}\left(\mathfrak{U}_{0}\right)$.

2.1.2. Definitions over $\mathfrak{X}_{0, \mathbb{Q}}$. We refer to [9, 1.1.1] for a basic discussion of the sheaf of algebraic differential operators over a smooth rigid analytic space such as $\mathfrak{X}_{0, \mathbb{Q}}$. It is defined in the following way, analogously to definition given in [12, 16.8] which we have recalled in 2.1.1. As before, $\overline{\mathcal{I}}_{\Delta}$ denotes the diagonal ideal of the immersion $\mathfrak{X}_{0, \mathbb{Q}} \hookrightarrow$ $\mathfrak{X}_{0, \mathbb{Q}} \times \mathfrak{X}_{0, \mathbb{Q}}$. One puts

$$
\mathcal{D}_{\mathfrak{X}_{0, \mathbb{Q}}}^{n}=\mathcal{H o m}_{\mathcal{O}_{\mathfrak{X}_{0, \mathbb{Q}}}}\left(\mathcal{O}_{\mathfrak{X}_{0, \mathbb{Q}} \times \mathfrak{X}_{0, \mathbb{Q}}} / \overline{\mathcal{I}}_{\Delta}^{n+1}, \mathcal{O}_{\mathfrak{X}_{0, \mathbb{Q}}}\right),
$$

which is a sheaf of locally free $\mathcal{O}_{\mathfrak{X}_{0, \mathbb{Q}}}$-modules of finite type and $\mathcal{D}_{\mathfrak{X}_{0, \mathbb{Q}}}=\underline{\lim }_{\rightarrow} \mathcal{D}_{\mathfrak{X}_{0, \mathbb{Q}}}^{n}$. The latter is a sheaf of rings acting naturally on the structure sheaf $\mathcal{O}_{\mathfrak{X}_{0, \mathbb{Q}}}$ of the rigid analyic space $\mathfrak{X}_{0, \mathbb{Q}}$.

Suppose now that

$$
\text { pr }: \mathfrak{X} \rightarrow \mathfrak{X}_{0}
$$

is an admissible blow-up of the formal scheme $\mathfrak{X}_{0}$ defined by a coherent sheaf of open ideals $\mathcal{I}$ of $\mathcal{O}_{\mathfrak{X}_{0}}$ containing $\varpi^{k}$. We remark that the ideal $\mathcal{I}$ is not determined by the blow-up pr : $\mathfrak{X} \rightarrow \mathfrak{X}_{0}$, i.e., different open ideal sheaves can give rise to isomorphic blow-ups. (For example, the blow-ups defined by $\mathcal{I}$ and by $\varpi^{n} \mathcal{I}$ are isomorphic as formal schemes over $\mathfrak{X}_{0}$. The same holds for the ideals $\mathcal{I}$ and $\mathcal{I}^{r}$.) In the sequel we denote by $k_{\mathcal{I}}$ the minimal $k$ such that $\varpi^{k} \in \mathcal{I}$ and put

$$
k_{\mathfrak{X}}=\min \left\{k_{\mathcal{I}} \mid \mathfrak{X} \text { is the blowing-up of } \mathcal{I} \text { on } \mathfrak{X}_{0}\right\} .
$$


Let us define now for $k \geqslant k_{\mathfrak{X}}$ the $\mathcal{O}_{\mathfrak{X}}$-module

$$
\mathcal{D}_{\mathfrak{X}}^{(k, m)}=\operatorname{pr}^{*} \mathcal{D}_{\mathfrak{X}_{0}}^{(k, m)} .
$$

We have the following commutative diagram of ringed spaces, involving the specialization maps $\mathfrak{X}_{0, \mathbb{Q}} \rightarrow \mathfrak{X}_{0}$ and $\mathfrak{X}_{\mathbb{Q}} \rightarrow \mathfrak{X}$, which we denote both by sp, and the isomorphism $\tilde{p r}: \mathfrak{X}_{\mathbb{Q}} \stackrel{\simeq}{\longrightarrow} \mathfrak{X}_{0, \mathbb{Q}}$ induced by the morphism pr on generic fibres:

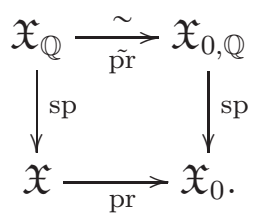

Note that $\mathcal{D}_{\mathfrak{X}_{\mathbb{Q}}} \simeq \tilde{\operatorname{pr}} * \mathcal{D}_{\mathfrak{X}_{0, \mathbb{Q}}}$ for the corresponding sheaves of differential operators on $\mathfrak{X}_{\mathbb{Q}}$ respectively $\mathfrak{X}_{0, \mathbb{Q}}$, as follows from the definition of these sheaves.

Lemma 2.1.6.

(i) There is a canonical isomorphism $\mathcal{D}_{\mathfrak{X}_{0, \mathbb{Q}}} \simeq \mathrm{sp}^{*} \mathcal{D}_{\mathfrak{X}_{0}}$ inducing an injective morphism of sheaves of rings

$$
\mathcal{D}_{\mathfrak{X}_{0}} \hookrightarrow \operatorname{sp}_{*} \mathcal{D}_{\mathfrak{X}_{0, \mathbb{Q}}} .
$$

(ii) There is a canonical injective map of sheaves of abelian groups

$$
\mathcal{D}_{\mathfrak{X}}^{(k, m)} \hookrightarrow \operatorname{sp}_{*} \mathcal{D}_{\mathfrak{X}_{\mathbb{Q}}},
$$

which becomes an isomorphism upon tensoring with $\mathbb{Q}$.

Proof. We have a canonical map $\mathcal{O}_{\mathfrak{X}, \mathbb{Q}} \rightarrow \operatorname{sp}_{*} \mathcal{O}_{\mathfrak{X} \mathbb{Q}}$, that is locally an isomorphism over the formal scheme $\mathfrak{X}$ (resp. $\mathfrak{X}_{0}$ ) and is thus an isomorphism of sheaves. Let us begin by (i). We have a canonical map $\mathcal{D}_{\mathfrak{X}_{0, \mathbb{Q}}} \rightarrow \mathrm{sp}^{*} \mathcal{D}_{\mathfrak{X}_{0}}$. To check that this is an isomorphism, we can work locally on $\mathfrak{X}_{0}$ and assume that $\mathfrak{X}_{0}$ is affine, endowed with local coordinates $x_{1}, \ldots, x_{M}$. Then, using notations 2.1 .2 we see that both sheaves are free $\mathcal{O}_{\mathfrak{X}_{0, \mathbb{Q}}}$-modules with basis $\underline{\partial}^{\underline{\nu}}$ and $\underline{\partial}^{[\underline{ }]}$ respectively. The previous map takes $\underline{\partial}^{\underline{\nu}}$ to $\underline{\partial}^{[\underline{\nu}} \underline{\nu}$ ! and is an isomorphism of sheaves of $\mathcal{O}_{\mathfrak{X}_{0, \mathbb{Q}}}$-modules. We obtain the second map of (i) by adjunction and its injectivity follows again using local coordinates. As in [12, 16.8.9], the ring structure on both sheaves makes use of the descriptions 2.1.1, resp. 2.1.4. That the map $\mathcal{D}_{\mathfrak{X}_{0}} \hookrightarrow \mathrm{sp}_{*} \mathcal{D}_{\mathfrak{X}_{0, \mathbb{Q}}}$ is compatible with ring structures comes then from the fact that $\operatorname{sp}^{*}\left(\mathcal{I}_{\Delta}\right) \simeq \overline{\mathcal{I}}_{\Delta}$ and

$$
\operatorname{sp}^{*}\left(\mathcal{O}_{\mathfrak{X}_{0}} \otimes \mathcal{O}_{\mathfrak{X}_{0}} / \mathcal{I}_{\Delta}^{n+1}\right) \simeq \mathcal{O}_{\mathfrak{X}_{0, \mathbb{Q}}} \otimes \mathcal{O}_{\mathfrak{X}_{0, \mathbb{Q}}} / \overline{\mathcal{I}}_{\Delta}^{n+1}
$$

Let us prove (ii). From the previous isomorphism, we get an isomorphism

$$
\mathrm{sp}^{*} \operatorname{pr}^{*} \mathcal{D}_{\mathfrak{X}_{0}} \simeq \tilde{\operatorname{pr}^{*}} \operatorname{sp}^{*} \mathcal{D}_{\mathfrak{X}_{0}} \simeq \tilde{\operatorname{pr}} * \mathcal{D}_{\mathfrak{X}_{0, \mathbb{Q}}} \simeq \mathcal{D}_{\mathfrak{X}_{\mathbb{Q}}}
$$

that induces a canonical map pr* $\mathcal{D}_{\mathfrak{X}_{0}} \rightarrow \operatorname{sp}_{*} \mathcal{D}_{\mathfrak{X}_{\mathbb{Q}}}$. Composing with the map $\mathcal{D}_{\mathfrak{X}_{0}}^{(k, m)} \rightarrow \mathcal{D}_{\mathfrak{X}_{0}}$ we get a map

$$
\operatorname{pr}^{*} \mathcal{D}_{\mathfrak{X}_{0}}^{(k, m)} \rightarrow \operatorname{sp}_{*} \mathcal{D}_{\mathfrak{X}_{\mathbb{Q}}}
$$


It is a local question to prove that this map is injective. Let $\mathfrak{U} \subset \mathfrak{X}_{0}$ be an open affine formal scheme of $\mathfrak{X}_{0}$ such that $\mathfrak{U} \subset \mathrm{pr}^{-1} \mathfrak{U}_{0}$, with $\mathfrak{U}_{0}$ endowed with local coordinates $x_{1}, \ldots, x_{M}$. Then these local coordinates give local coordinates always denoted $x_{1}, \ldots, x_{M}$ over the generic fiber $\mathfrak{U}_{\mathbb{Q}}$ of $\mathfrak{U}$. Using notations 2.1.2, the sheaf $\operatorname{pr}^{*} \mathcal{D}_{\mathfrak{U}_{0}}^{(k, m)}$ is a free $\mathcal{O}_{\mathfrak{U}^{-}}$ module with basis the operators $\left.\varpi^{k|\underline{\underline{ }}|} \underline{\partial} \underline{\underline{\nu}}\right\rangle$ whereas the sheaf $\operatorname{sp}_{*} \mathcal{D}_{\mathfrak{U}_{\mathbb{Q}}}$ is a free $\mathcal{O}_{\mathfrak{U}} \otimes \mathbb{Q}-$ module with basis $\underline{\partial}^{\underline{\nu}}$. The map we consider takes $\varpi^{k \mid \underline{\nu}} \underline{\partial} \underline{\partial}^{\underline{\nu}\rangle}$ to $\varpi^{k \mid \underline{\nu}} q_{\nu} ! / \underline{\nu} ! \underline{\partial} \underline{\underline{\nu}}$. Since $\mathfrak{U}$ is flat over $\mathfrak{o}$, the map $\mathcal{O}_{\mathfrak{U}} \rightarrow \mathcal{O}_{\mathfrak{U}} \otimes \mathbb{Q}$ is injective, and this proves that the canonical map pr* $\mathcal{D}_{\mathfrak{X}_{0}}^{(k, m)} \rightarrow \operatorname{sp}_{*} \mathcal{D}_{\mathfrak{X}_{\mathbb{Q}}}$ is injective as well. The same argument shows that this map becomes an isomorphism upon tensoring with $\mathbb{Q}$.

Following [22], given $k \geqslant k_{\mathfrak{X}}$, we will construct a $p$-adically complete sheaf of arithmetic differential operators $\widehat{\mathcal{D}}_{\mathfrak{X}}^{(k, m)}$ over the (usually non-smooth) formal scheme $\mathfrak{X}$.

2.1.3. Construction of the ring of differential operators of level $k$ over $\mathfrak{X}$. We first observe that the sheaf $\operatorname{sp}_{*} \mathcal{D}_{\mathfrak{X}_{\mathbb{Q}}}$ acts on $\operatorname{sp}_{*} \mathcal{O}_{\mathfrak{X}_{\mathbb{Q}}} \simeq \mathcal{O}_{\mathfrak{X}, \mathbb{Q}}$. By 2.1.6, the sheaf $\mathcal{D}_{\mathfrak{X}}^{(k, m)}$ is a subsheaf of $\operatorname{sp}_{*} \mathcal{D}_{\mathfrak{X}_{\mathbb{Q}}}$ and $\mathcal{D}_{\mathfrak{X}, \mathbb{Q}}^{(k, m)} \simeq \operatorname{sp}_{*} \mathcal{D}_{\mathfrak{X}_{\mathbb{Q}}}$. We will first check that if $k \geqslant k_{\mathfrak{X}}$, the action of $\operatorname{sp}_{*} \mathcal{D}_{\mathfrak{X}_{\mathbb{Q}}}$ on $\mathcal{O}_{\mathfrak{X}, \mathbb{Q}}$ restricts to an action of $\mathcal{D}_{\mathfrak{X}}^{(k, m)}$ on $\mathcal{O}_{\mathfrak{X}}$. This can be checked locally on $\mathfrak{X}$. For this, we assume that $\mathfrak{X}_{0}=\operatorname{Spf} A$, where $A$ is a smooth, complete, o-algebra, endowed with local coordinates $x_{1}, \ldots, x_{M}$. Since $\mathfrak{X}_{0}$ is smooth over Spfo, both rings $A$ and $A / \varpi A$ are integral domains. We also introduce the differential operators $\underline{\partial}^{\langle\underline{\nu}\rangle}$ and $\varpi^{k \mid \underline{\nu}} \underline{\underline{\partial}} \underline{\underline{\nu}} \underline{ }$ according to 2.1 .2 , of $D^{(m)}=\Gamma\left(\mathfrak{X}_{0}, \mathcal{D}_{\mathfrak{X}_{0}}^{(m)}\right)$ and $D^{(k, m)}=\Gamma\left(\mathfrak{X}_{0}, \mathcal{D}_{\mathfrak{X}_{0}}^{(k, m)}\right)$ respectively. We also denote $I=\Gamma\left(\mathfrak{X}_{0}, \mathcal{I}\right)$ where $V(\mathcal{I})$ is the center of the blowing-up $\mathfrak{X}$.

Consider the $\mathbb{N}$-graded $A$-algebra

$$
B=\bigoplus_{n} B_{n}
$$

where the degree $n$-part $B_{n}$ equals the $n$-th power $I^{n}$ of the ideal $I$. In particular, $I^{0}=A$. This means that

$$
\mathfrak{X}=\widehat{\operatorname{Proj}}(B) \text {, }
$$

the formal completion of $\operatorname{Proj}(B)$. The algebra $B$ is integral, as $A$ is integral. Let $t \in B_{D}$ be a homogeneous element of degree $D>0$, and let $C_{t}=B[1 / t]_{0}$ be the algebra of degree 0 elements in the homogeneous localization $B[1 / t]$. Then $C_{t}$ is non-zero, since $B$ is integral. Put $D_{+}(t)=\operatorname{Spf} \widehat{C}_{t}$. These open sets form a basis for the Zariski topology of $\mathfrak{X}$.

Let us observe that the algebra $B$ is a graded subalgebra of $A[T]$. Indeed, there is a graded injective ring morphism

$$
\begin{gathered}
B \stackrel{\varphi}{\longrightarrow} A[T] \\
x_{n} \in B_{n} \longrightarrow x_{n} T^{n} .
\end{gathered}
$$


By definition, $\varphi(t)=t T^{D}$. Since localization is flat, we get from this an injective graded morphism $B[1 / t] \rightarrow A[1 / t]\left[T^{ \pm 1}\right]$, where $A[1 / t]\left[T^{ \pm 1}\right]$ is graded by the degree of $T$. Because $C_{t}=B[1 / t]_{0}$ is the subring of degree zero elements in $B[1 / t]$, we get an injection

$$
C_{t} \hookrightarrow A[1 / t]
$$

Since $A\{1 / t\}=\Gamma\left(D(t), \mathcal{O}_{\mathfrak{X}_{0}}\right)$, and because $\mathcal{D}_{\mathfrak{X}_{0}}^{(k, m)}$ acts on the structural sheaf $\mathcal{O}_{\mathfrak{X}_{0}}$, we get that $A\{1 / t\}$ is a $D^{(m)}$-module and thus a $D^{(k, m)}$-module. Moreover, as $A[1 / t]$ is integral and noetherian, it embeds into its $p$-adic completion $A\{1 / t\}$. This leads us to the following

\section{Lemma 2.1.8.}

(i) $A[1 / t]$ is a $D^{(m)}$-submodule of $A\{1 / t\}$,

(ii) If $k \geqslant k_{\mathfrak{X}}$, then $C_{t}$ is a $D^{(k, m)}$-submodule of $A[1 / t]$.

Proof. Before giving the proof, we need some notation. Given a fixed nonnegative integer $m$ and a nonnegative integer $\nu$, one denotes as before by $q$ the quotient of the Euclidean division of $\nu$ by $p^{m}$. Let $\nu \geqslant \nu^{\prime}$ be two nonnegative integers and $\nu^{\prime \prime}:=\nu-\nu^{\prime}$; then for the corresponding numbers $q, q^{\prime}$ and $q^{\prime \prime}$, we define

$$
\left\{\begin{array}{c}
\nu \\
\nu^{\prime}
\end{array}\right\}=\frac{q !}{q^{\prime} ! q^{\prime \prime} !}
$$

which is an integer because $q \geqslant q^{\prime}+q^{\prime \prime}$. Let us begin with the proof of (i). It is enough to prove that for each $i \leqslant M$, for each invertible $s \in A[1 / t]$, for each $\nu \in \mathbb{N}$,

$$
\partial_{i}^{\langle\nu\rangle}\left(s^{-1}\right) \in \frac{A}{s^{\nu+1}} .
$$

We will prove this by induction on $\nu$, the case $\nu=0$ being straightforward. We have

$$
\partial_{i}^{\langle\nu+1\rangle}\left(s^{-1}\right)=-\sum_{\mu=0}^{\nu}\left\{\begin{array}{c}
\nu+1 \\
\mu
\end{array}\right\} s^{-1} \partial_{i}^{\langle\nu+1-\mu\rangle}(s) \partial_{i}^{\langle\mu\rangle}\left(s^{-1}\right) .
$$

cf. [6, (iv) of 2.2.4]. By induction hypothesis the elements $\partial_{i}^{\langle\mu\rangle}\left(s^{-1}\right)$ lie in $s^{-(\nu+1)} A$, which proves that $\partial_{i}^{\langle\nu+1\rangle}\left(s^{-1}\right) \in s^{-(\nu+2)} A$. By applying this to $s=t^{-n}$ for $n>0$, we see that (i) holds.

Let us prove now (ii). We begin the proof with an auxiliary assertion (it is here where we use the assumption $k \geqslant k_{\mathfrak{X}}$ ).

Assertion. Let $f \in I^{r}, l \in \mathbb{N}$, then $\varpi^{k l} \partial_{i}^{\langle l\rangle}(f) \in I^{r}$.

Proof of the assertion. The proof relies on the Leibniz formula [6, 2.3.4.1]. We proceed by induction on $r$. For $r=0$ the assertion is trivial and for $r=1$, it is true if $l \geqslant 1$ since $\varpi^{k} \in I$. For $r=1$, it is also true if $l=0$ since $f \in I$. Let us assume that the result is true for $s \leqslant r$. It is enough to prove that

$$
\forall g \in I \forall h \in I^{r}: \varpi^{k l} \partial_{i}^{\langle l\rangle}(h g) \in I^{r+1} .
$$


Denote $f=h g$, the Leibniz formula of loc. cit. states that

$$
\varpi^{k l} \partial_{i}^{\langle l\rangle}(f)=\sum_{j=0}^{l}\left\{\begin{array}{l}
l \\
j
\end{array}\right\} \varpi^{k j} \partial_{i}^{\langle j\rangle}(h) \varpi^{k(l-j)} \partial_{i}^{\langle l-j\rangle}(g) .
$$

By induction hypothesis, for all $j \leqslant l, \varpi^{k j} \partial_{i}^{\langle j\rangle}(h) \in I^{r}$ and $\varpi^{k(l-j)} \partial_{i}^{\langle l-j\rangle}(g) \in I$, which implies that $\varpi^{k l} \partial_{i}^{\langle l\rangle}(f) \in I^{r+1}$. This establishes the assertion.

After this preliminary discussion, let $d>0$. Let us first prove by induction on $\nu$ that for an arbitrary element $s \in I^{d}$ which becomes invertible in $A[1 / t]$, one has

$$
\varpi^{k \nu} \partial_{i}^{\langle\nu\rangle}\left(s^{-1}\right) \in \frac{I^{\nu d}}{s^{\nu+1}} .
$$

This is true for $\nu=0$. Consider then the formula [6, (iv) of 2.2.4] with notation 2.1.9]

$$
\varpi^{k(\nu+1)} \partial_{i}^{\langle\nu+1\rangle}\left(s^{-1}\right)=-\sum_{\mu=0}^{\nu}\left\{\begin{array}{c}
\nu+1 \\
\mu
\end{array}\right\} s^{-1} \varpi^{k(\nu+1-\mu)} \partial_{i}^{\langle\nu+1-\mu\rangle}(s) \varpi^{k \mu} \partial_{i}^{\langle\mu\rangle}\left(s^{-1}\right) .
$$

By the induction hypothesis, one knows for any integer $\mu \leqslant \nu$,

$$
\varpi^{k \mu} \partial_{i}^{\langle\mu\rangle}\left(s^{-1}\right) \in \frac{I^{\mu d}}{s^{\mu+1}}
$$

and, by our auxiliary assertion above, one knows

This implies

$$
s^{-1} \varpi^{k(\nu+1-\mu)} \partial_{i}^{\langle\nu+1-\mu\rangle}(s) \in \frac{I^{d}}{s} .
$$

$$
s^{-1} \varpi^{k(\nu+1-\mu)} \partial_{i}^{\langle\nu+1-\mu\rangle}(s) \varpi^{k \mu} \partial_{i}^{\langle\mu\rangle}\left(s^{-1}\right) \in \frac{I^{d}}{s} \frac{I^{\mu d}}{s^{\mu+1}}=\frac{I^{d(\mu+1)}}{s^{\mu+2}}=\frac{s^{\nu-\mu} I^{d(\mu+1)}}{s^{\nu+2}} \subseteq \frac{I^{(\nu+1) d}}{s^{\nu+2}},
$$

which proves our claim. Applying this claim to the element $s=t^{d} \in I^{d D}$ gives for $\mu \leqslant p^{m}$

$$
\varpi^{k \mu} \partial_{i}^{\langle\mu\rangle}\left(t^{-d}\right) \in \frac{I^{\mu d D}}{t^{d(\mu+1)}} .
$$

Then, using again the Leibniz formula, we deduce from this and the auxiliary assertion, for a given homogeneous element $g \in B$ of degree $d D$, i.e $\frac{g}{t^{d}} \in C_{t}$, the identity

$$
\varpi^{k \nu} \partial_{i}^{\langle\nu\rangle}\left(\frac{g}{t^{d}}\right)=\sum_{\mu=0}^{\nu}\left\{\begin{array}{l}
\nu \\
\mu
\end{array}\right\} \varpi^{k(\nu-\mu)} \partial_{i}^{\langle\nu-\mu\rangle}(g) \varpi^{k \mu} \partial_{i}^{\langle\mu\rangle}\left(t^{-d}\right)
$$

whose right-hand terms are contained in

$$
I^{d D} \frac{I^{\mu d D}}{t^{d(\mu+1)}} \subset C_{t}
$$

This completes the proof of the lemma.

From this we get the 
Corollary 2.1.11. $\widehat{C}_{t}$ is a $D^{(k, m)}$-submodule of $A\{1 / t\}_{\mathbb{Q}}$ if $k \geqslant k_{\mathfrak{X}}$.

Proof. Using previous notations, we see that $\varpi^{k|\underline{\nu}|} \partial_{i}^{\langle\underline{\nu}\rangle}$ acts continuously on $C_{t}$. We can thus extend this action by continuity to get an action on $\widehat{C}_{t}$. By construction this action is induced by the action of $\Gamma\left(\mathfrak{X}_{0}, \operatorname{sp}_{*} \mathcal{D}_{\mathfrak{X}_{0, \mathbb{Q}}}\right)$ on $\left.\widehat{A\{1 / t}\right\}_{\mathbb{Q}}$.

After these local considerations, we come back now to the general situation.

Corollary 2.1.12. Let $k \geqslant k_{\mathfrak{X}}$. The sheaf $\mathcal{D}_{\mathfrak{X}}^{(k, m)}=\operatorname{pr}^{*} \mathcal{D}_{\mathfrak{X}_{0}}^{(k, m)}$ is a subsheaf of rings of the sheaf $\operatorname{sp}_{*} \mathcal{D}_{\mathfrak{X}_{\mathbb{Q}}}$. Moreover it is locally free over $\mathcal{O}_{\mathfrak{X}}$.

Proof. The assertion is local on $\mathfrak{X}$ and we can assume that $\mathfrak{X}_{0}=\operatorname{Spf} A$ is affine, endowed

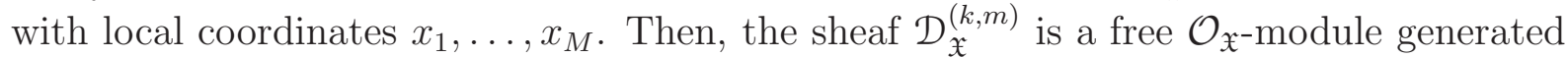

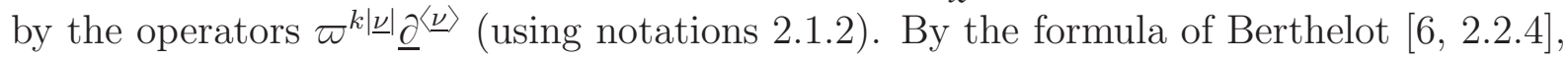
one has

$$
\underline{\partial}^{\langle\underline{\nu}\rangle} \cdot \underline{\partial}^{\left\langle\underline{\nu}^{\prime}\right\rangle}=\left\langle\begin{array}{c}
\underline{\nu}+\underline{\nu}^{\prime} \\
\underline{\nu}
\end{array}\right\rangle \underline{\partial}^{\left\langle\underline{\nu}+\underline{\nu}^{\prime}\right\rangle}
$$

where

$$
\left\langle\begin{array}{c}
\underline{\nu}+\underline{\nu^{\prime}} \\
\underline{\nu}
\end{array}\right\rangle=\left(\begin{array}{c}
\underline{\nu}+\underline{\nu}^{\prime} \\
\underline{\nu}
\end{array}\right)\left\{\begin{array}{c}
\underline{\nu}+\underline{\nu}^{\prime} \\
\underline{\nu}
\end{array}\right\}^{-1} \in \mathbb{Z}_{p}
$$

To check that $\mathcal{D}_{\mathfrak{X}}^{(k, m)}$ is a subsheaf of rings of $\operatorname{sp}_{*} \mathcal{D}_{\mathfrak{X}_{\mathbb{Q}}}$, we thus only have to check, by linearity, that if $b$ is a section in $\mathcal{O}_{\mathfrak{X}}(\mathfrak{V})$ where $\left.\mathfrak{V}=\operatorname{Spf} \widehat{C_{t}[1 / h}\right]$, cf. 2.1.11, for some non zero $t \in A$, and non zero $h \in C_{t}$, and if $\underline{\nu} \in \mathbb{N}^{M}$, then the element $\underline{\partial} \underline{\underline{\nu}\rangle} \cdot b$ lies in $\mathcal{D}_{\mathfrak{X}}^{(k, m)}(\mathfrak{V})$. By [6, 2.2.4], one has

$$
\varpi^{k|\underline{\nu}|} \underline{\partial}\langle\underline{\nu}\rangle \cdot b=\sum_{\underline{\nu}^{\prime}+\underline{\nu}^{\prime \prime}=\underline{\nu}}\left\{\begin{array}{l}
\underline{\nu} \\
\underline{\nu}^{\prime}
\end{array}\right\} \varpi^{k\left|\underline{\nu}^{\prime}\right|} \underline{\partial}^{\left\langle\underline{\nu}^{\prime}\right\rangle}(b) \varpi^{k\left|\underline{\nu}^{\prime \prime}\right|} \underline{\partial}^{\left\langle\underline{\nu}^{\prime \prime}\right\rangle} .
$$

Since $\varpi^{k\left|\underline{\nu}^{\prime}\right|} \underline{\partial}^{\left\langle\underline{\nu}^{\prime}\right\rangle}(b) \in \mathcal{O}_{\mathfrak{X}}(\mathfrak{V})$ by 2.1 .11 , this proves that $\mathcal{D}_{\mathfrak{X}}^{(k, m)}$ is a subring of $\mathcal{D}_{\mathfrak{X}_{\mathbb{Q}}}$.

We finally define the following sheaves of differential operators over $\mathfrak{X}$ and $X_{i}$

$$
\mathcal{D}_{X_{i}}^{(k, m)}=\mathcal{D}_{\mathfrak{X}}^{(k, m)} / \varpi^{i+1}, \widehat{\mathcal{D}}_{\mathfrak{X}}^{(k, m)}={\underset{\lim }{\leftarrow}}_{\mathcal{D}_{X_{i}}^{(k, m)}} \text { and } \mathcal{D}_{\mathfrak{X}, k}^{\dagger}=\underset{m}{\lim } \widehat{\mathcal{D}}_{\mathfrak{X}}^{(k, m)} \otimes \mathbb{Q} \text {. }
$$

We have the following local description over an affine open $\mathfrak{V} \subseteq \operatorname{pr}^{-1}\left(\mathfrak{U}_{0}\right)$ where $\mathfrak{U}_{0}$ is an affine open of $\mathfrak{X}_{0}$ endowed with coordinates $x_{1}, \ldots, x_{M}$ and derivations $\partial_{1}, \ldots, \partial_{M}$ :

$$
\mathcal{D}_{\mathfrak{X}, k}^{\dagger}(\mathfrak{V})=\left\{\sum_{\underline{\nu}} a_{\underline{\nu}} \varpi^{k|\underline{\nu}|} \underline{\partial}^{[\underline{\nu}]} \mid a_{\underline{\underline{ }}} \in \mathcal{O}_{\mathfrak{X}, \mathbb{Q}}(\mathfrak{V}), \text { and } \exists C>0, \eta<1|| a_{\underline{\underline{\nu}}} \mid<C \eta^{|\underline{\nu}|}\right\},
$$

where $|$.$| denotes any Banach norm on \mathcal{O}_{\mathfrak{X}, \mathbb{Q}}(\mathfrak{V})$. 
2.2. First properties. We keep here the hypothesis from the previous section. In particular, $\mathfrak{X}_{0}$ denotes a smooth formal $\mathfrak{S}$-scheme and

$$
\text { pr }: \mathfrak{X} \rightarrow \mathfrak{X}_{0}
$$

denotes an admissible blow-up. For a given natural number $k \geqslant 0$ we let

$$
\mathcal{T}_{\mathfrak{X}, k}:=\varpi^{k}(\mathrm{pr})^{*}\left(\mathcal{T}_{\mathfrak{X}_{0}}\right),
$$

where $\mathcal{T}_{\mathfrak{X}_{0}}$ is the relative tangent sheaf of $\mathfrak{X}_{0}$ over $\mathfrak{S}$.

\section{Lemma 2.2.1.}

(i) The sheaf $\mathcal{T}_{\mathfrak{X}, k}$ is a locally free $\mathcal{O}_{\mathfrak{X}}$-module of rank equal to the relative dimension of $\mathfrak{X}_{0}$ over $\mathfrak{S}$.

(ii) Suppose $\pi: \mathfrak{X}^{\prime} \rightarrow \mathfrak{X}$ is a morphism over $\mathfrak{X}_{0}$ from another admissible blow-up $\mathrm{pr}^{\prime}: \mathfrak{X}^{\prime} \rightarrow \mathfrak{X}_{0}$. Let $k^{\prime}, k \geqslant 0$. One has as subsheaves of $\mathcal{T}_{\mathfrak{X}^{\prime}} \otimes L$

$$
\mathcal{T}_{\mathfrak{X}^{\prime}, k^{\prime}}=\varpi^{k^{\prime}-k} \pi^{*}\left(\mathcal{T}_{\mathfrak{X}, k}\right)
$$

Proof. This follows directly from the definitions. Note that $\left(\mathrm{pr}^{\prime}\right)^{*}=\pi^{*} \circ \mathrm{pr}^{*}$ in (ii).

Before stating the next proposition, let us recall that $\operatorname{Sym}^{(m)}\left(\mathcal{T}_{\mathfrak{X}, k}\right)$ denotes the graded level $m$ symmetric algebra generated by the sheaf $\mathcal{T}_{\mathfrak{X}, k}$ defined in [16, sec. 1]. This is a graded $\mathcal{O}_{\mathfrak{X} \text {-algebra }}$

$$
\operatorname{Sym}^{(m)}\left(\mathcal{T}_{\mathfrak{X}, k}\right)=\bigoplus_{d \geqslant 0} \operatorname{Sym}_{d}^{(m)}\left(\mathcal{T}_{\mathfrak{X}, k}\right)
$$

Over some sufficiently small open affine set $\mathfrak{U} \subseteq \operatorname{pr}^{-1}\left(\mathfrak{U}_{0}\right)$ such that $\mathcal{T}_{\mathfrak{U}_{0}}$ is free with basis $\xi_{1}, \ldots, \xi_{M}$, one has the description using notation 2.1.2 (i.e. $\left.\nu ! \xi_{l}^{\langle\nu\rangle}=q_{\nu} ! \xi_{l}^{\nu}\right)$

$$
\operatorname{Sym}_{d}^{(m)}\left(\mathcal{T}_{\mathfrak{X}, k}\right)(\mathfrak{U})=\underset{|\underline{\nu}|=d}{\bigoplus} \mathcal{O}_{\mathfrak{X}}(\mathfrak{U}) \varpi^{k d} \underline{\xi}^{\langle\underline{\nu}\rangle},
$$

where the right hand side is a free $\mathcal{O}_{\mathfrak{X}}(\mathfrak{U})$-module. For the rest of this subsection we fix a number $k \geqslant k_{\mathfrak{X}}(2.1 .5)$.

\section{Proposition 2.2.2.}

(i) The sheaves $\mathcal{D}_{\mathfrak{X}}^{(k, m)}$ are filtered by the sheaves of differential operators of order

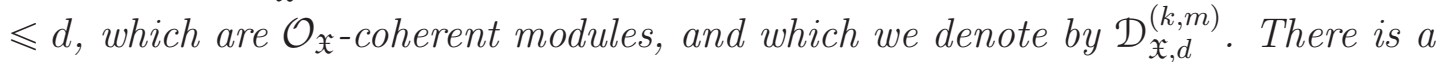
canonical isomorphism of sheaves of graded algebras

$$
\operatorname{gr}\left(\mathcal{D}_{\mathfrak{X}}^{(k, m)}\right) \simeq \operatorname{Sym}^{(m)}\left(\mathcal{T}_{\mathfrak{X}, k}\right)
$$

Moreover the sheaves $\mathcal{D}_{X_{i}}^{(k, m)}$ are quasi-coherent $\mathcal{O}_{X_{i}}$-modules.

(ii) There is a basis of the topology $\mathcal{B}$ of $\mathfrak{X}$ (resp. $X_{i}$ ), consisting of open affine subsets, such that for any $\mathfrak{U} \in \mathcal{B}$ (resp. $\left.U_{i} \in \mathcal{B}\right)$, the ring $\mathcal{D}_{\mathfrak{X}}^{(k, m)}(\mathfrak{U})\left(\operatorname{resp} . \mathcal{D}_{X_{i}}^{(k, m)}\left(U_{i}\right)\right.$ ) is two-sided noetherian. 
(iii) For every formal affine open $\mathfrak{U} \subseteq \mathfrak{X}$ (resp. affine open $U_{i} \subseteq X_{i}$ ), the ring $\mathcal{D}_{\mathfrak{X}}^{(k, m)}(\mathfrak{U})$ (resp. $\mathcal{D}_{X_{i}}^{(k, m)}\left(U_{i}\right)$ ) is two-sided noetherian.

(iv) The sheaves of rings $\mathcal{D}_{\mathfrak{X}}^{(k, m)}$ (resp. $\mathcal{D}_{X_{i}}^{(k, m)}$ ) are coherent.

(v) For every formal affine open $\mathfrak{U} \subset \mathfrak{X}$, the ring $\widehat{\mathcal{D}}_{\mathfrak{X}}^{(k, m)}(\mathfrak{U})$ is two-sided noetherian.

(vi) The sheaf of rings $\widehat{\mathcal{D}}_{\mathfrak{X}}^{(k, m)}$ is coherent.

Proof. We only do the proof of (i) to (iv) in the case of $\mathcal{D}_{\mathfrak{X}}^{(k, m)}$, since the same proof works for the sheaf $\mathcal{D}_{X_{i}}^{(k, m)}$. Denote by $\mathcal{D}_{\mathfrak{X}_{0}, d}^{(k, m)}$ the sheaf of differential operators of $\mathcal{D}_{\mathfrak{X}_{0}}^{(k, m)}$ of order $\leqslant d$, and $\mathcal{D}_{\mathfrak{X}, d}^{(k, m)}=\operatorname{pr}^{*} \mathcal{D}_{\mathfrak{X}_{0}, d}^{(k, m)}$. It is straightforward that we have an exact sequence of $\mathcal{O}_{\mathfrak{X}_{0}}$-modules on $\mathfrak{X}_{0}$

$$
0 \longrightarrow \mathcal{D}_{\mathfrak{X}_{0}, d-1}^{(k, m)} \longrightarrow \mathcal{D}_{\mathfrak{X}_{0}, d}^{(k, m)} \longrightarrow \operatorname{Sym}_{d}^{(m)}\left(\mathcal{T}_{\mathfrak{X}_{0}, k}\right) \longrightarrow 0
$$

Now we apply pr* and get an exact sequence since $\operatorname{Sym}_{d}^{(m)}\left(\mathcal{T}_{\mathfrak{X}_{0}, k}\right)$ is a locally free $\mathcal{O}_{\mathfrak{X}_{0}}$ module of finite rank. This gives (i). Let $\mathfrak{U}$ be an affine subset of $\operatorname{pr}^{-1}\left(\mathfrak{U}_{0}\right)$, where $\mathfrak{U}_{0} \subset \mathfrak{X}_{0}$ has some coordinates $x_{1}, \ldots, x_{M}$. One has the following description

$$
\mathcal{D}_{\mathfrak{X}}^{(k, m)}(\mathfrak{U})=\left\{\sum_{\underline{\nu}}^{<\infty} \varpi^{k|\underline{\nu}|} a_{\underline{\nu}} \underline{\partial}^{\langle\underline{\nu}\rangle} \mid a_{\underline{\nu}} \in \mathcal{O}_{\mathfrak{X}}(\mathfrak{U})\right\} .
$$

Since $\mathfrak{U}$ is affine and the filtration steps $\mathcal{D}_{\mathfrak{x}, d}^{(k, m)}$ are coherent $\mathcal{O}_{\mathfrak{X}}$-modules for all $d$, the previous exact sequences gives us the following isomorphism

$$
\operatorname{gr}\left(\mathcal{D}_{\mathfrak{X}}^{(k, m)}\right)(\mathfrak{U}) \simeq \operatorname{Sym}_{\mathcal{O}_{\mathfrak{X}}}^{(m)}\left(\mathcal{T}_{\mathfrak{X}, k}\right)(\mathfrak{U})
$$

Since the latter level $m$ symmetric algebra is known to be noetherian [16, Prop. 1.3.6], this proves (ii). As $\mathcal{B}$ we may take the set of open affine subsets of $\mathfrak{X}$ that are contained in some $\operatorname{pr}^{-1}\left(\mathfrak{U}_{0}\right)$, for some open $\mathfrak{U}_{0} \subset \mathfrak{X}_{0}$ endowed with global coordinates. Let now $\mathfrak{V}, \mathfrak{U} \in \mathcal{B}$ such that $\mathfrak{V} \subset \mathfrak{U}$. Since the sheaf $\mathcal{D}_{\mathfrak{X}}^{(k, m)}$ is an inductive limit of coherent $\mathcal{O}_{\mathfrak{X} \text {-modules, one has }}$

$$
\mathcal{O}_{\mathfrak{X}}(\mathfrak{V}) \otimes_{\mathcal{O}_{\mathfrak{X}}(\mathfrak{U})} \mathcal{D}_{\mathfrak{X}}^{(k, m)}(\mathfrak{U}) \simeq \mathcal{D}_{\mathfrak{X}}^{(k, m)}(\mathfrak{V})
$$

In particular $\mathcal{D}_{\mathfrak{X}}^{(k, m)}(\mathfrak{V})$ is flat over $\mathcal{D}_{\mathfrak{X}}^{(k, m)}(\mathfrak{U})$. This remark and (ii) prove the coherence of the sheaves $\mathcal{D}_{\mathfrak{X}}^{(k, m)}$ exactly as in the proof [6, 3.1.3].

Let us now prove (iii) for 'left noetherian' (the proof of the right version is similar). Let $\mathfrak{U}$ be an affine open of $\mathfrak{X}, \mathcal{D}=\mathcal{D}_{\mathfrak{U}}^{(k, m)}, D=\Gamma(\mathfrak{U}, \mathcal{D}), A=\Gamma\left(\mathfrak{U}, \mathcal{O}_{\mathfrak{X}}\right)$ and $\mathfrak{U}=\bigcup \mathfrak{U}_{l}$ a finite cover of $\mathfrak{U}$ by open $\mathfrak{U}_{l} \in \mathcal{B}$. Since the sheaf $\mathcal{D}$ is an inductive limit of coherent $\mathcal{O}_{\mathfrak{X}}$-modules, one has

$$
\mathcal{D}=\mathcal{O}_{\mathfrak{U}} \otimes_{A} D
$$


and $\mathcal{D}$ is a flat $D$-module. Moreover, thanks to (ii), we know that $\mathcal{D}_{\mathfrak{X}}\left(\mathfrak{U}_{l}\right)$ is noetherian for each $l$. Let $\left(M_{i}\right)$ be an increasing sequence of left ideals of $D$, and consider

$$
\mathcal{M}_{i}=\mathcal{D} \otimes_{D} M_{i}=\mathcal{O}_{\mathfrak{U}} \otimes_{A} M_{i},
$$

which form an increasing sequence of sheaves of ideals of $\mathcal{D}$ by flatness of $\mathcal{D}$ over $D$. The sequence $\Gamma\left(\mathfrak{U}_{l}, \mathcal{M}_{i}\right)$ is thus an increasing sequence of ideals of $\Gamma\left(\mathfrak{U}_{l}, \mathcal{D}\right)$, that is stationary since this algebra is noetherian. Since $M_{i}$ is an inductive limit of finite type $A$-modules, $\mathcal{M}_{i}$ is an inductive limit of coherent $\mathcal{O}_{\mathfrak{X}}$-modules, thus

$$
\forall l, \mathcal{M}_{i \mid \mathfrak{U}_{l}} \simeq \mathcal{O}_{\mathfrak{U}_{l}} \otimes_{\mathcal{O}_{\mathfrak{X}}\left(\mathfrak{U}_{l}\right)} \Gamma\left(\mathfrak{U}_{l}, \mathcal{M}_{i}\right) \text { and } \Gamma\left(\mathfrak{U}, \mathcal{M}_{i}\right)=M_{i}
$$

Finally we see that $\mathcal{M}_{i \mid \mathfrak{U}_{l}}$ is stationary for each $l$. Since there are finitely many affine open $\mathfrak{U}_{l}$, we see that the sequence $\left(\mathcal{M}_{i}\right)$ and thus $\left(M_{i}\right)$ are stationary. This proves (iii). Since $\widehat{\mathcal{D}}_{\mathfrak{X}}^{(k, m)}(\mathfrak{U})$ is the $p$-adic completion of $\widehat{\mathcal{D}}_{\mathfrak{X}}^{(k, m)}(\mathfrak{U})$, it is also left and right noetherian [6. 3.2.3], which proves (v).

The coherence of $\widehat{\mathcal{D}}_{\mathfrak{X}}^{(k, m)}$ follows from (iii), and the fact that $\mathcal{D}_{X_{i}}^{(k, m)}$ is a quasi-coherent $\mathcal{O}_{X_{i}}$-module, literally as in [6, 3.3.3].

From these considerations, and under our initial condition $k \geqslant k_{\mathfrak{X}}$, we have the following local versions of Cartan's Theorem A and B for the restrictions of the sheaves $\mathcal{D}_{X_{i}}^{(k, m)}$ and $\widehat{\mathcal{D}}_{\mathfrak{X}}^{(k, m)}$ to an open affine (formal) subscheme.

Proposition 2.2.6. (Local theorem A and B for fixed $m$ )

(i) Let $U_{i} \subset X_{i}$ be an open affine subscheme of $X_{i}$. The functor $\Gamma\left(U_{i},.\right)$ establishes an equivalence of categories between coherent $\mathcal{D}_{U_{i}}^{(k, m)}$-modules and finite type $\Gamma\left(U_{i}, \mathcal{D}_{X_{i}}^{(k, m)}\right)$-modules. In particular, the functor $\Gamma\left(U_{i},.\right)$ is exact on coherent $\mathcal{D}_{U_{i}}^{(k, m)}$-modules. Moreover, for any coherent $\mathcal{D}_{U_{i}}^{(k, m)}$-module $\mathcal{M}$ and any $q>0$ one has $H^{q}\left(U_{i}, \mathcal{M}\right)=0$.

(ii) Let $\mathfrak{U} \subset \mathfrak{X}$ be an open affine formal subscheme of $\mathfrak{X}$. The functors $\mathcal{M} \mapsto \Gamma(\mathfrak{U}, \mathcal{M})$ and $M \mapsto \widehat{\mathcal{D}}_{\mathfrak{U}}^{(k, m)} \otimes M$ are quasi-inverse equivalences of categories between the category of coherent left $\widehat{\mathcal{D}}_{\mathfrak{U}}^{(k, m)}$-modules and the category of left $\Gamma\left(\mathfrak{U}, \widehat{\mathcal{D}}_{\mathfrak{U}}^{(k, m)}\right)$-modules of finite type.

(iii) Let $\mathfrak{U}$ be as in (ii). The functor $\Gamma(\mathfrak{U},$.$) is exact on coherent \widehat{\mathcal{D}}_{\mathfrak{U}}^{(k, m)}$-modules. Moreover, for any coherent $\widehat{\mathcal{D}}_{\mathfrak{U}}^{(k, m)}$-module $\mathcal{M}$ and any $q>0$ one has $H^{q}(\mathfrak{U}, \mathcal{M})=$ 0 .

Note that (ii) of the proposition remains true for the sheaf $\widehat{\mathcal{D}}_{\mathfrak{U}, \mathbb{Q}}^{(k, m)}$ and coherent modules over this sheaf by [6, 3.4.6].

Proof. For the convenience of the reader, we start by recalling the following result

Auxiliary result 2.2.7. (cf. [6, Prop. 3.1.3]) Let $X$ be a scheme, $\mathcal{D}$ be a sheaf of rings over $X$ such that, for all affine open $U \subset X, \Gamma(U, \mathcal{D})$ is a noetherian ring. We fix an 
homomorphism $\mathcal{O}_{X} \rightarrow \mathcal{D}$ such that the left multiplication by the sections of $\mathcal{O}_{X}$ induces a structure of $\mathcal{O}_{X}$-coherent ring over $\mathcal{D}$.

(i) The sheaf $\mathcal{D}$ is a left coherent sheaf of rings.

(ii) A left $\mathcal{D}$-module $\mathcal{M}$ is coherent if and only if it is a quasi-coherent $\mathcal{O}_{X}$-module and, for all affine open $U$ of an affine cover of $X, \Gamma(U, \mathcal{M})$ is a left $\Gamma(U, \mathcal{D})$-module of finite type.

(iii) Assume that $X$ is affine and let $D=\Gamma(X, \mathcal{D})$. The functors $\mathcal{M} \mapsto \Gamma(X, \mathcal{M})$ and $M \mapsto \mathcal{D} \otimes M$ are quasi-inverse equivalences of categories between the category of coherent left $\mathcal{D}$-modules and the category of left $D$-modules of finite type.

Consider now the following situation, compare [6, 3.3.3]. Let $\mathfrak{X}^{\prime}$ be an $\mathfrak{S}$-formal scheme and let $\mathcal{D}$ be a sheaf of rings over $\mathfrak{X}^{\prime}$, endowed with a homomorphism $\mathcal{O}_{\mathfrak{X}^{\prime}} \rightarrow \mathcal{D}, \mathcal{D}_{i}=$ $\mathcal{D} / p^{i+1}, \widehat{\mathcal{D}}=\lim _{i} \mathcal{D}_{i}$. In addition, assume the following conditions (Berthelot's conditions)

(1) As an $\mathcal{O}_{\mathfrak{X}^{\prime}}$-module, $\mathcal{D}$ is filtered inductive limit of a family of $\mathcal{O}_{\mathfrak{X}^{\prime}}$-module $\mathcal{D}_{\lambda}$ such that $\mathcal{D}_{\lambda} / p^{i} \mathcal{D}_{\lambda}$ are $\mathcal{O}_{X_{i}^{\prime}}$-quasi-coherent and $\mathcal{D}_{\lambda} \simeq \lim _{i} \mathcal{D}_{\lambda} / p^{i} \mathcal{D}_{\lambda}$.

(2) For every open set $\mathfrak{U} \subset \mathfrak{X}^{\prime}$, the ring $\Gamma(\mathfrak{U}, \mathcal{D})$ is left noetherian.

Auxiliary result 2.2.8. With the previous hypotheses, suppose that $\mathfrak{X}^{\prime}$ is affine, and let $\widehat{D}=\Gamma\left(\mathfrak{X}^{\prime}, \widehat{\mathcal{D}}\right)$. Then $\widehat{D}$ is left noetherian. If $M$ is a $\widehat{D}$-module one defines a $\widehat{\mathcal{D}}$-module

$$
M^{\Delta}=\lim _{i} \mathcal{D}_{i} \otimes_{\mathcal{O}_{X_{i}^{\prime}}} M / p^{i+1} M
$$

For a $\widehat{\mathcal{D}}$-module $\mathcal{M}$, the following statements are equivalent

(i) For all $i$, the $\mathcal{D}_{i}$-module $\mathcal{M} / p^{i+1} \mathcal{M}$ is coherent and the canonical homomorphism $\mathcal{M} \rightarrow \lim _{i} \mathcal{M} / p^{i+1} \mathcal{M}$ is an isomorphism.

(ii) There exists an isomorphism $\mathcal{M} \simeq \lim _{i} \mathcal{M}_{i}$, where $\left(\mathcal{M}_{i}\right)$ is a projective system of coherent $\mathcal{D}_{i}$-modules, such that the transition morphisms factorize via isomorphisms $\mathcal{M}_{i} / p^{i} \mathcal{M}_{i} \simeq \mathcal{M}_{i-1}$.

(iii) There exists a finite type $\widehat{D}$-module $M$ and an isomorphism $\mathcal{M} \simeq M^{\Delta}$.

(iv) $\mathcal{M}$ is a coherent $\widehat{\mathcal{D}}$-module.

Proof. The ring $\widehat{D}$ is noetherian by [6, Prop. 3.3.4] and the other results come from [6, Prop. 3.3.9].

Under the same hypotheses, Berthelot proves in addition the following.

Auxiliary result 2.2.9. (cf. [6, Prop. 3.3.10/11]) With the previous hypotheses, suppose that $\mathfrak{X}^{\prime}$ is affine. The functors $\Gamma\left(\mathfrak{X}^{\prime}, \cdot\right)$ and $M \mapsto M^{\Delta}$ are equivalences between the category of coherent $\widehat{\mathcal{D}}$-modules and the category of finite type $\widehat{D}$-modules. If $\mathcal{M}$ is a coherent $\widehat{\mathcal{D}}$-module, then $\forall q \geqslant 1, H^{q}\left(\mathfrak{X}^{\prime}, \mathcal{M}\right)=0$.

Now it is clear that part (i) of our proposition 2.2.6 follows from auxiliary result 2.2.7, since by (iii) of 2.2.2, the rings $\mathcal{D}_{X_{i}}^{(k, m)}\left(U_{i}\right)$ are indeed noetherian. Again, from (iii) of 2.2 .2 , 
we see that the rings $\mathcal{D}_{\mathfrak{X}}^{(k, m)}(\mathfrak{U})$ are noetherian. Moreover the sheaf $\mathcal{D}_{\mathfrak{X}}^{(k, m)}$ is a filtered inductive limit of the $\mathcal{O}_{\mathfrak{X}}$-coherent sheaves $\mathcal{D}_{\mathfrak{X}, d}^{(k, m)}$ defined in the proof of 2.2.2. This means that Berthelot's conditions (1) and (2) are satisfied for $\mathfrak{X}^{\prime}=\mathfrak{X}$ and $\mathcal{D}=\mathcal{D}_{\mathfrak{X}}^{(k, m)}$. Hence, the auxiliary results 2.2.8 and 2.2.9 can be applied in our context, proving (ii) of the proposition. The point (iii) is a direct consequence of (ii). This ends the proof of the proposition 2.2.6.

Proposition 2.2.10. Let $\mathfrak{U} \subset \mathfrak{X}$ be an open affine formal subscheme of $\mathfrak{X}$, and $\mathcal{M}$ a coherent $\widehat{\mathcal{D}}_{\mathfrak{U}}^{(k, m)}$-module. Then there are integers $a, b \geqslant 0$ and a short exact sequence of coherent $\widehat{\mathcal{D}}_{\mathfrak{U}}^{(k, m)}$-modules:

$$
\left(\widehat{\mathcal{D}}_{\mathfrak{U}}^{(k, m)}\right)^{a} \rightarrow\left(\widehat{\mathcal{D}}_{\mathfrak{U}}^{(k, m)}\right)^{b} \rightarrow \mathcal{M} \rightarrow 0
$$

Proof. Denote $\widehat{D}^{(m)}=\Gamma\left(\mathfrak{U}, \widehat{\mathcal{D}}_{\mathfrak{U}}^{(k, m)}\right)$ which is a noetherian ring by 2.2 .2 and $M=\Gamma(\mathfrak{U}, \mathcal{M})$, which is a finite type $\widehat{D}^{(m)}$-module by the previous proposition 2.2.6. Since the algebra $\widehat{D}^{(m)}$ is noetherian, there exists a finite presentation of $\widehat{D}^{(m)}$-modules

$$
\left(\widehat{D}^{(m)}\right)^{a} \rightarrow\left(\widehat{D}^{(m)}\right)^{b} \rightarrow M \rightarrow 0
$$

Tensoring this presentation by $\widehat{\mathcal{D}}_{\mathfrak{U}}^{(k, m)}$ over the ring $\widehat{D}^{(m)}$ and observing that

$$
\mathcal{M} \simeq \widehat{\mathcal{D}}_{\mathfrak{U}^{(k, m)}} \otimes_{\widehat{D}^{(m)}} M
$$

again by 2.2.6, gives the result.

Proposition 2.2.11. We have:

(i) The morphism of sheaves $\widehat{\mathcal{D}}_{\mathfrak{X}, \mathbb{Q}}^{(k, m)} \rightarrow \widehat{\mathcal{D}}_{\mathfrak{X}, \mathbb{Q}}^{(k, m+1)}$ is left and right flat.

(ii) The sheaf of rings $\mathcal{D}_{\mathfrak{X}, k}^{\dagger}$ is coherent.

(iii) For any affine open $\mathfrak{U} \subset \mathfrak{X}, \Gamma\left(\mathfrak{U}, \widehat{\mathcal{D}}_{\mathfrak{X}, \mathbb{Q}}^{(k, m+1)}\right)$ is left and right flat over $\Gamma\left(\mathfrak{U}, \widehat{\mathcal{D}}_{\mathfrak{X}, \mathbb{Q}}^{(k, m)}\right)$.

(iv) For any affine open $\mathfrak{U} \subset \mathfrak{X}, \Gamma\left(\mathfrak{U}, \mathcal{D}_{\mathfrak{X}, k}^{\dagger}\right)$ is left and right flat over $\Gamma\left(\mathfrak{U}, \widehat{\mathcal{D}}_{\mathfrak{X}, \mathbb{Q}}^{(k, m)}\right)$.

Proof. Let us first observe that (ii) follows from the flatness statement of (i) and the last part of 2.2.2 thanks to [6, 3.6.1]. For (i), we follow Berthelot's method described in [6, 3.5.3]. For the proof we can restrict ourselves to proving that if $\mathfrak{U}$ is an affine open of $\mathfrak{X}$ contained in the basis of open sets $\mathcal{B}$ from 2.2.2, then the map $\widehat{\mathcal{D}}_{\mathfrak{X}, \mathbb{Q}}^{(k, m)}(\mathfrak{U}) \rightarrow \widehat{\mathcal{D}}_{\mathfrak{X}, \mathbb{Q}}^{(k, m+1)}(\mathfrak{U})$ is left and right flat. In this situation, we have the following explicit description, assuming that $\operatorname{pr}^{*} \mathcal{T}_{\mathfrak{X}_{0}}$ is free restricted to $\mathfrak{U}$, with basis $\partial_{1}, \ldots, \partial_{M}$ as in 2.1.14,

$$
\mathcal{D}_{\mathfrak{X}}^{(k, m)}(\mathfrak{U})=\left\{\sum_{\underline{\nu}}^{<\infty} \varpi^{k|\underline{\nu}|} b_{\underline{\nu}} \underline{\partial}^{\langle\underline{\nu}\rangle} \mid b_{\underline{\nu}} \in B\right\} \subset \widehat{\mathcal{D}}_{\mathfrak{X}}^{(k, m)}(\mathfrak{U})=\left\{\sum_{\underline{\nu}} \varpi^{k|\underline{\nu}|} b_{\underline{\nu}} \underline{\partial}^{\langle\underline{\nu}\rangle} \mid b_{\underline{\nu}} \in B, b_{\underline{\nu}} \rightarrow 0\right\}
$$

where the convergence is in the $\varpi$-adic topology of $B=\mathcal{O}_{\mathfrak{X}}(\mathfrak{U})$. With this description, we can copy Berthelot's proof of [ $\underline{6}, 3.5 .3]$, replacing everywhere the operators $\underline{\partial}^{\langle\underline{\nu}\rangle}$ by 
$\varpi^{k \mid \underline{\nu}} \underline{\partial} \underline{\nu}^{\underline{\nu}\rangle}$, as follows. First of all, by inserting suitable powers of $\varpi$ into the formula [6, (2.2.5.1)] we see that the $\mathfrak{o}$-algebra $\mathcal{D}_{\mathfrak{X}}^{(k, m)}(\mathfrak{U})$ is generated by $B$ and the operators $\varpi^{k p^{r}} \partial_{l}^{\left[p^{r}\right]}$ for $1 \leqslant r \leqslant m$ and $1 \leqslant l \leqslant M$. Now write

$$
D^{(k, m)}=\mathcal{D}_{\mathfrak{X}}^{(k, m)}(\mathfrak{U}), \text { and } \widehat{D}^{(k, m)}=\widehat{\mathcal{D}}_{\mathfrak{X}}^{(k, m)}(\mathfrak{U}),
$$

the latter being the $\varpi$-adic completion of $D^{(k, m)}$ [6, 4.3.3.2]. By the above explicit description, the two canonical maps $D^{(k, m)} \rightarrow \widehat{D}^{(k, m)} \rightarrow \widehat{D}_{\mathbb{Q}}^{(k, m)}$ are injective and this is also true for the canonical map $\widehat{D}^{(k, m)} \rightarrow \widehat{D}^{(k, m+1)}$. Indeed, the latter is induced by mapping

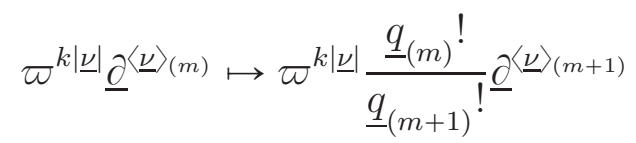

and is therefore injective by the above explicit description and the fact that $\mathfrak{o}$ is torsionfree. Let us consider the subring $E$ of $\widehat{D}_{\mathbb{Q}}^{(k, m)}$ generated by the subsets $\widehat{D}^{(k, m)}$ and $D^{(k, m+1)}$. Since $D_{\mathbb{Q}}^{(k, m+1)}=D_{\mathbb{Q}}^{(k, m)}$, we see that

$$
E_{\mathbb{Q}}=\widehat{D}_{\mathbb{Q}}^{(k, m)} .
$$

As in Berthelot's proof, we have the following

Auxiliary result 2.2.13. $E=\widehat{D}^{(k, m)}+D^{(k, m+1)}$.

Proof. Denote by $E^{\prime}=\widehat{D}^{(k, m)}+D^{(k, m+1)}$. We have to prove that $E^{\prime}$ is a subalgebra of $\widehat{D}^{(k, m+1)}$. This is enough to prove that if $(P, Q) \in \widehat{D}^{(k, m)} \times D^{(k, m+1)}$, then $P \cdot Q \in E^{\prime}$ and $Q \cdot P \in E^{\prime}$. Since the proof is the same in both cases, we only treat the product $P \cdot Q$. As $Q \in D^{(k, m+1)}$, there exists $c>0$ such that $\varpi^{c} Q \in D^{(k, m)}$. As $P \in \widehat{D}^{(k, m)}$, there exist $\left(P_{1}, R_{1}\right) \in D^{(k, m)} \times \widehat{D}^{(k, m)}$ such that $P=P_{1}+\varpi^{c} R_{1}$, then $P Q=\varpi^{c} R_{1} Q+P_{1} Q \in E^{\prime}$, as $\varpi^{c} R_{1} Q \in \widehat{D}^{(k, m)}$ and $P_{1} Q \in D^{(k, m+1)}$.

Denote by $\widehat{E}$ the $\varpi$-adic completion of $E$. We can then prove the

Auxiliary result 2.2.14. $\widehat{E}_{\mathbb{Q}}=\widehat{D}_{\mathbb{Q}}^{(k, m+1)}$.

Proof. By construction, there are maps

$$
\lambda_{i}: D^{(k, m+1)} / \varpi^{i} D^{(k, m+1)} \rightarrow E / \varpi^{i} E .
$$

Let us prove that these maps are bijective. If $R \in D^{(k, m+1)}$ is such that $\lambda_{i}(R) \in \varpi^{i} E$ then there exist $(P, Q) \in \widehat{D}^{(k, m)} \times D^{(k, m+1)}$ such that $R=\varpi^{i}(P+Q)$, thus $\varpi^{i} P=R-\varpi^{i} Q$ has finite order, and $P \in D^{(k, m)}$, that implies that $R \in D^{(k, m+1)}$ and $\lambda_{i}$ is injective. Pick now $R \in E$, and $(P, Q) \in \widehat{D}^{(k, m)} \times D^{(k, m+1)}$ such that $R=P+Q$. The operator $P$ can be written $P=P_{1}+\varpi^{i} R_{1}$, with $P_{1} \in D^{(k, m)}$ and $R_{1} \in \widehat{D}^{(k, m)}$, then $P=P_{1}+R+\varpi^{i} R_{1}$ and $\lambda_{i}\left(P_{1}+R \bmod \varpi^{i} D^{(k, m+1)}\right)=P \bmod \varpi^{i} E$, so that $\lambda_{i}$ is surjective. We finally see that $\lambda_{i}$ is bijective, which proves the auxiliary result. 
Now, the remaining thing to prove is that $E$ is noetherian, since this result implies that $\widehat{E}$ is flat over $E$, thus that $\widehat{D}_{\mathbb{Q}}^{(k, m+1)}$ is flat over $\widehat{D}_{\mathbb{Q}}^{(k, m)}$. The proof that $E$ is noetherian proceeds by induction. By our above remark, $E$ is generated as (left) $D^{(k, m)}$-module by the elements $\varpi^{k|\underline{\nu}| p^{m+1}}\left(\underline{\partial}^{\left[p^{m+1}\right]}\right) \underline{\nu}$ for $\underline{\nu} \in \mathbb{N}^{M}$. Let $1 \leqslant l \leqslant M$. Inserting appropriate powers of $\varpi$ into the corresponding formula in Berthelot's proof one finds $\left[\varpi^{k p^{m+1}} \partial_{l}^{\left[p^{m+1}\right]}, b\right] \in D^{(k, m)}$ for any $b \in B$ and so $\left[\varpi^{k p^{m+1}} \partial_{l}^{\left[p^{m+1}\right]}, P\right] \in \widehat{D}^{(k, m)}$ for any $P \in \widehat{D}^{(k, m)}$. Using the general commutator identity (valid in any associative ring) $\left[Q^{\nu}, P\right]=\left[Q^{\nu-1}, P\right] Q+Q^{\nu-1}[Q, P]$ one deduces from this inductively

$$
\left[\left(\varpi^{k p^{m+1}} \partial_{l}^{\left[p^{m+1}\right]}\right)^{\nu}, P\right] \in \sum_{\mu<\nu} \widehat{D}^{(k, m)}\left(\varpi^{k p^{m+1}} \partial_{l}^{\left[p^{m+1}\right]}\right)^{\mu}
$$

This is the analogue of the key formula [6, (3.5.3.2)]. Now let $1 \leqslant j \leqslant M$ and consider the subring $E_{j}$ of $E$ generated by $E_{0}=\widehat{D}^{(k, m)}$ and the operators $\varpi^{k p^{m+1}} \partial_{l}^{\left[p^{m+1}\right]}$ for $1 \leqslant l \leqslant j$. Then $E_{0}$ is noetherian by prop. 2.2 .2 and, by our above discussion, $E_{M}=E$. With the key formula at hand, one may now follow Berthelot's proof word for word to obtain that $E_{j-1}$ noetherian implies $E_{j}$ noetherian. This proves (i).

Let us now prove (iii). Denote $\widehat{D}_{k, \mathbb{Q}}^{(m)}=\Gamma\left(\mathfrak{U}, \widehat{\mathcal{D}}_{\mathfrak{U}, \mathbb{Q}}^{(k, m)}\right)$ and consider $\alpha$ an injective map of coherent $\widehat{D}_{k, \mathbb{Q}}^{(m)}$-modules $\alpha: M \hookrightarrow N$. As a consequence of (ii) and (iii) of 2.2.6, we know that the sheaf $\widehat{\mathcal{D}}_{\mathfrak{U}, \mathbb{Q}}^{(k, m)}$ is a flat $\widehat{D}_{k, \mathbb{Q}}^{(m)}$-module. In particular, the map $\alpha$ provides an injection of coherent $\widehat{\mathcal{D}}_{\mathfrak{U}, \mathbb{Q}}^{(k, m)}$-modules

$$
\widehat{\mathcal{D}}_{\mathfrak{U}, \mathbb{Q}}^{(k, m)} \otimes_{\hat{D}_{k, \mathbb{Q}}^{(m)}} M \hookrightarrow \widehat{\mathcal{D}}_{\mathfrak{U}, \mathbb{Q}}^{(k, m)} \otimes_{\hat{D}_{k, \mathbb{Q}}^{(m)}} N
$$

Using the flatness result (i) we also have an injection of coherent $\widehat{\mathcal{D}}_{\mathfrak{U}, \mathbb{Q}}^{(k, m+1)}$-modules

$$
\widehat{\mathcal{D}}_{\mathfrak{U}, \mathbb{Q}}^{(k, m+1)} \otimes_{\widehat{D}_{k, \mathbb{Q}}^{(m)}} M \hookrightarrow \widehat{\mathcal{D}}_{\mathfrak{U}, \mathbb{Q}}^{(k, m+1)} \otimes_{\widehat{D}_{k, \mathbb{Q}}^{(m)}} N
$$

Then we identify (resp. for $N$ )

$$
\widehat{\mathcal{D}}_{\mathfrak{U}, \mathbb{Q}}^{(k, m+1)} \otimes_{\widehat{D}_{k, \mathbb{Q}}^{(m)}} M \simeq \widehat{\mathcal{D}}_{\mathfrak{U}, \mathbb{Q}}^{(k, m+1)} \otimes_{\widehat{D}_{k, \mathbb{Q}}^{(m+1)}} \widehat{D}_{k, \mathbb{Q}}^{(m+1)} \otimes_{\widehat{D}_{k, \mathbb{Q}}^{(m)}} M .
$$

Finally taking global sections of the previous injection and using again 2.2.6, we get an injection of coherent $\widehat{D}_{k, \mathbb{Q}}^{(m+1)}$-modules

$$
\widehat{D}_{k, \mathbb{Q}}^{(m+1)} \otimes_{\widehat{D}_{k, \mathbb{Q}}^{(m)}} M \hookrightarrow \widehat{D}_{k, \mathbb{Q}}^{(m+1)} \otimes_{\widehat{D}_{k, \mathbb{Q}}^{(m)}} N,
$$

that proves (iii). Assertion (iv) follows from the previous one, since, as $\mathfrak{U}$ is quasi-compact,

$$
\Gamma\left(\mathfrak{U}, \mathcal{D}_{\mathfrak{X}, k}^{\dagger}\right)=\underset{m}{\lim } \Gamma\left(\mathfrak{U}, \widehat{\mathcal{D}}_{\mathfrak{X}, \mathbb{Q}}^{(k, m)}\right),
$$


and we deduce from (iii) that, for all integer $m^{\prime} \geqslant m$, the module $\Gamma\left(\mathfrak{U}, \widehat{\mathcal{D}}_{\mathfrak{X}, \mathbb{Q}}^{\left(k, m^{\prime}\right)}\right)$ is left and right flat over $\Gamma\left(\mathfrak{U}, \widehat{\mathcal{D}}_{\mathfrak{X}, \mathbb{Q}}^{(k, m)}\right)$. We obtain thus (iv) by passing to the inductive limit over $m$. This ends the proof of the proposition 2.2.11.

We deduce from this the corresponding version of proposition 2.2.6 for the sheaf $\mathcal{D}_{\mathfrak{X}, k}^{\dagger}$.

Corollary 2.2.15. (Local theorem A and B for varying $m$ ) Let $\mathfrak{U} \subset \mathfrak{X}$ be an open affine formal subscheme of $\mathfrak{X}$. Then :

(i) The algebra $D_{k}^{\dagger}=\Gamma\left(\mathfrak{U}, \mathcal{D}_{\mathfrak{X}, k}^{\dagger}\right)$ is coherent.

(ii) For any open affine subset $\mathfrak{U} \subset \mathfrak{X}$, any coherent $\mathcal{D}_{\mathfrak{U}, k}^{\dagger}$-module $\mathcal{M}$, and any $q>0$ one has $H^{q}(\mathfrak{U}, \mathcal{M})=0$.

(iii) The functor $\Gamma(\mathfrak{U},$.$) establishes an equivalence of categories between coherent \mathcal{D}_{\mathfrak{U}, k^{-}}^{\dagger}$ modules and coherent $D_{k}^{\dagger}$-modules. In particular, the functor $\Gamma(\mathfrak{U},$.$) is exact on$ coherent $\mathcal{D}_{\mathfrak{U}, k}^{\dagger}$-modules.

Proof. Denote $\widehat{D}_{k, \mathbb{Q}}^{(m)}=\Gamma\left(\mathfrak{U}, \widehat{\mathcal{D}}_{\mathfrak{U}, \mathbb{Q}}^{(k, m)}\right)$. Since the scheme $\mathfrak{U}$ is quasi-compact, the functors $H^{q}(\mathfrak{U},$.$) commute with inductive limits and we have$

$$
D_{k}^{\dagger}=\underset{m}{\lim } \widehat{D}_{k, \mathbb{Q}}^{(m)}
$$

By (iii) of the 2.2.11, the maps $\widehat{D}_{k, \mathbb{Q}}^{(m)} \rightarrow \widehat{D}_{k, \mathbb{Q}}^{(m+1)}$ are flat, and by 2.2 .2 these algebras $\widehat{D}_{k, \mathbb{Q}}^{(m)}$ are noetherian. This showes that the algebra $D_{k}^{\dagger}$ is coherent [6, 3.6.1].

Let $\mathcal{M}$ be a coherent $\mathcal{D}_{\mathfrak{U}, k}^{\dagger}$-module. The proof of [6, 3.6.2] literally applies in our situation and shows that there is a non-negative integer $m_{0}$ and a coherent $\widehat{\mathcal{D}}_{\mathfrak{U}, \mathbb{Q}}^{\left(k, m_{0}\right)}$-module $\mathcal{N}$ such that

$$
\mathcal{M} \simeq \mathcal{D}_{\mathfrak{U}, k}^{\dagger} \otimes_{\hat{\mathcal{D}}_{\mathfrak{U}, \mathbb{Q}}^{\left(k, m_{0}\right)}} \mathcal{N}
$$

Denote $M=\Gamma(\mathfrak{U}, \mathcal{M})$ and for $m \geqslant m_{0}$

$$
\mathcal{M}^{(m)}=\widehat{\mathcal{D}}_{\mathfrak{U}, \mathbb{Q}}^{(k, m)} \otimes_{\widehat{\mathcal{D}}_{\mathfrak{U}, \mathbb{Q}}^{\left(k, m_{0}\right)}} \mathcal{N}
$$

so that $\mathcal{M} \simeq \lim _{\longrightarrow} \mathcal{M}^{(m)}$. Then, $\forall m \geqslant m_{0}, H^{q}\left(\mathfrak{U}, \mathcal{M}^{(m)}\right)=0$ by 2.2.6, and by passing to the inductive limit we see that $H^{q}(\mathfrak{U}, \mathcal{M})=0$, which proves (ii).

The rest of the proof follows 2.3.7, 2.4.1, 2.4.2 of [20]. For the convenience of the reader, let us summmarize the arguments here. From 2.2.10, the $\widehat{\mathcal{D}}_{\mathfrak{U}, \mathbb{Q}}^{\left(k, m_{0}\right)}$-coherent module $\mathcal{N}$ admits a resolution

$$
\left(\widehat{\mathcal{D}}_{\mathfrak{U}}^{\left(k, m_{0}\right)}\right)^{a} \rightarrow\left(\widehat{\mathcal{D}}_{\mathfrak{U}}^{\left(k, m_{0}\right)}\right)^{b} \rightarrow \mathcal{N} \rightarrow 0
$$


Tensoring this resolution with the sheaf $\mathcal{D}_{\mathfrak{U}, k}^{\dagger}$ gives us a resolution of coherent $\mathcal{D}_{\mathfrak{L}, k^{-}}^{\dagger}$ modules. Since the global section functor is exact on the category of coherent $\mathcal{D}_{\mathfrak{L}, k^{-}}^{\dagger}$ modules because of (ii), we get an exact sequence of coherent $D_{k}^{\dagger}$-modules

$$
\left(D_{k}^{\dagger}\right)^{a} \rightarrow\left(D_{k}^{\dagger}\right)^{b} \rightarrow M \rightarrow 0
$$

To see that $\mathcal{D}_{k}^{\dagger} \otimes_{D_{k}^{\dagger}} M \simeq \mathcal{M}$, we are thus reduced to the case $\mathcal{M}=\mathcal{D}_{\mathfrak{U}, k}^{\dagger}$, for which it is obvious. We prove similarly that if $M$ is a $D_{k}^{\dagger}$-coherent module, then

$$
M \simeq \Gamma\left(\mathfrak{U}, \mathcal{D}_{\mathfrak{U}, k}^{\dagger} \otimes_{D_{k}^{\dagger}} M\right),
$$

by reducing to the case where $M=D_{k}^{\dagger}$. This proves the proposition.

We now give a flatness result when the congruence niveau $k$ varies.

Proposition 2.2.16. Let $k, k^{\prime}$ be nonnegative integers such that $k^{\prime} \geqslant k \geqslant k_{\mathfrak{X}}$, then the morphism of sheaves of rings $\widehat{\mathcal{D}}_{\mathfrak{X}, \mathbb{Q}}^{\left(k^{\prime}, m\right)} \hookrightarrow \widehat{\mathcal{D}}_{\mathfrak{X}, \mathbb{Q}}^{(k, m)}$ is left and right flat.

Proof. By induction, it is enough to prove that the morphism $\widehat{\mathcal{D}}_{\mathfrak{X}, \mathbb{Q}}^{(k+1, m)} \hookrightarrow \widehat{\mathcal{D}}_{\mathfrak{X}, \mathbb{Q}}^{(k, m)}$ is left and right flat. It is also enough to prove the following statement : if $\mathfrak{U}$ is an affine open of the basis of open sets $\mathcal{B}$ from [2.2.2, then the map $\widehat{\mathcal{D}}_{\mathfrak{X}, \mathbb{Q}}^{(k+1, m)}(\mathfrak{U}) \hookrightarrow \widehat{\mathcal{D}}_{\mathfrak{X}, \mathbb{Q}}^{(k, m)}(\mathfrak{U})$ is left and right flat. Denote $D_{k}=\Gamma\left(\mathfrak{U}, \mathcal{D}_{\mathfrak{X}}^{(k, m)}\right)$ and $\widehat{D}_{k}=\Gamma\left(\mathfrak{U}, \widehat{\mathcal{D}}_{\mathfrak{X}}^{(k, m)}\right)$ (resp. with $\left.k+1\right)$. In our situation, we have the following explicit description (resp. with $k+1$ ), where $B=\Gamma\left(\mathfrak{U}, \mathcal{O}_{\mathfrak{X}}\right)$, assuming that $\operatorname{pr}^{*} \mathcal{T}_{\mathfrak{X}_{0}}$ is free restricted to $\mathfrak{U}$, with basis $\partial_{1}, \ldots, \partial_{M}$ as in 2.1.14,

$$
D_{k}=\left\{\sum_{\underline{\nu}}^{<\infty} \varpi^{k|\underline{\underline{\nu}}|} b_{\underline{\underline{\nu}} \underline{\underline{\partial}}} \underline{\underline{\nu}\rangle} \mid b_{\underline{\underline{\nu}}} \in B\right\} \quad \text { and } \quad \hat{D}_{k}=\left\{\sum_{\underline{\underline{\nu}}} \varpi^{k \mid \underline{\mid \underline{ }}} b_{\underline{\underline{\nu}} \underline{\underline{\partial}} \underline{\underline{\nu}}\rangle} \mid b_{\underline{\underline{\nu}}} \in B, b_{\underline{\underline{\nu}}} \rightarrow 0\right\} .
$$

Here, convergence is with respect to the $p$-adic topology on $B$. Moreover we have the inclusion $D_{k} \subset \widehat{D}_{k}$. As in the proof of the proposition 2.2.11 above, we will use Berthelot's method [6, 3.5.3], and introduce

$$
E=\widehat{D}_{k+1}+D_{k} .
$$

Then, since $D_{k, \mathbb{Q}}=D_{k+1, \mathbb{Q}}$, it is clear that $E_{\mathbb{Q}}=\widehat{D}_{k+1, \mathbb{Q}}$. Moreover, we have the following Auxiliary result 2.2.17. The $B$-module $E$ is a $B$-algebra and its $p$-adic completion $\hat{E}$ is canonically isomorphic to $\widehat{D}_{k}$.

Proof. Let us first prove that $E$ is a ring. Any element $P$ of $E$ can be written as

$$
P=Q+R, \text { with } Q \in \widehat{D}_{k+1} \text { and } R \in D_{k} .
$$

To prove that $E$ is a ring, it is enough to prove that

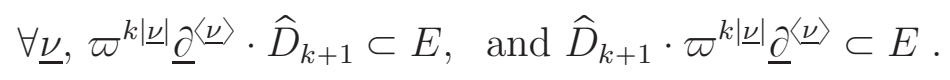


Fix $\underline{\nu}$, and take $Q \in \widehat{D}_{k+1}$. We can then write $Q=Q_{1}+\varpi^{|\underline{\mid}|} Q_{2}$, with $Q_{1} \in D_{k+1}$ and $Q_{2} \in \widehat{D}_{k+1}$. Since $\varpi^{k|\underline{\nu}|} \underline{\partial}^{\langle\underline{\nu}\rangle} \cdot Q_{1} \in D_{k}$, and $Q_{1} \cdot \varpi^{k|\underline{\nu}|} \underline{\partial}^{\langle\underline{\nu}\rangle} \in D_{k}$, it remains to prove that $\varpi^{k|\underline{\nu}|} \underline{\partial}^{\langle\underline{\nu}\rangle} \cdot \varpi^{|\underline{\underline{ }}|} Q_{2} \in E$ (resp. $\left.\varpi^{|\underline{\underline{ }}|} Q_{2} \cdot \varpi^{k \mid \underline{\nu}} \underline{\partial}^{\langle\underline{\nu}\rangle} \in E\right)$. Let us write

$$
Q_{2}=\sum_{\underline{\nu}^{\prime}} \varpi^{(k+1)\left|\underline{u}^{\prime}\right|} b_{\underline{\nu}^{\prime}}{\underline{\partial \underline{\nu^{\prime}}}}^{\langle},
$$

with coefficients $b_{\underline{\nu^{\prime}}} \in B$ tending $p$-adically to zero in $B$. Besides the coefficients appearing in 2.1.9, we need other modified binomial coefficients [6, (1.1.2.2)]

$$
\left\langle\begin{array}{l}
\underline{\nu} \\
\underline{\nu^{\prime}}
\end{array}\right\rangle=\left(\begin{array}{l}
\underline{\nu} \\
\underline{\nu^{\prime}}
\end{array}\right)\left\{\begin{array}{l}
\underline{\nu} \\
\underline{\nu}^{\prime}
\end{array}\right\}^{-1} \in \mathbb{Z}_{p}
$$

Then, following [6, (2.2.4)] we have the following formulas

$$
\begin{aligned}
& \varpi^{k|\underline{\nu}|} \underline{\partial}^{\langle\underline{\nu}\rangle} \cdot \varpi^{\left((k+1)\left|\underline{\nu}^{\prime}\right|+|\underline{\nu}|\right)} b_{\underline{\nu}^{\prime}} \underline{\partial}^{\left\langle\underline{\nu}^{\prime}\right\rangle} \\
& \left.=\varpi^{(k+1)\left(|\underline{\mid}|+\left|\underline{\nu}^{\prime}\right|\right)} \sum_{\underline{\mu} \leqslant \underline{\nu}}\{\underline{\underline{\mu}}\}\left\langle\underline{\underline{\nu}}+\underline{\nu}^{\prime}-\underline{\mu}\right\rangle \underline{\partial} \underline{\underline{\mu}}\right\rangle\left(b_{\underline{\nu^{\prime}}}\right) \underline{\partial}^{\left\langle\underline{\nu}+\underline{\nu}^{\prime}-\underline{\mu}\right\rangle} \in \widehat{D}_{k+1} .
\end{aligned}
$$

Passing to the limit in the complete ring $\widehat{D}_{k+1}$ we see that $\varpi^{k|\underline{\nu}|} \underline{\partial} \underline{\underline{\nu}\rangle} \cdot \varpi^{|\underline{\nu}|} Q_{2} \in \widehat{D}_{k+1}$. Similarly, we have

$$
\varpi^{\left((k+1)\left|\underline{\underline{\prime}}^{\prime}\right|+|\underline{\nu}|\right)} b_{\underline{\nu}^{\prime}} \underline{\partial}^{\left\langle\underline{\nu}^{\prime}\right\rangle} \cdot \varpi^{k|\underline{\nu}|} \underline{\partial}^{\langle\underline{\nu}\rangle}=b_{\underline{\nu}^{\prime}} \varpi^{\left((k+1)\left(\left|\underline{\nu}^{\prime}\right|+|\underline{\nu}|\right)\right.}\left\langle\begin{array}{c}
\left.\underline{\nu}+\underline{\nu}^{\prime}\right\rangle \\
\underline{\nu}
\end{array}\right\rangle \underline{\partial}^{\left\langle\underline{\nu}+\underline{\nu}^{\prime}\right\rangle},
$$

which proves that $\varpi^{|\underline{\nu}|} Q_{2} \cdot \varpi^{k \mid \underline{\nu}} \underline{\partial} \underline{\underline{\nu}}^{\underline{ }\rangle} \in E$ and that $E$ is a ring.

Let $i \geqslant 0$ be an integer and consider now the canonical map

$$
\lambda: D_{k} / \varpi^{i} D_{k} \rightarrow E / \varpi^{i} E .
$$

Let $P \in E$, and $Q \in \widehat{D}_{k+1}, R \in D_{k}$ such that $P=Q+R$. There exist $Q_{1} \in D_{k+1}$ and $Q_{2} \in \widehat{D}_{k+1}$ such that $Q=Q_{1}+\varpi^{i} Q_{2}$. Then

$$
Q=\lambda\left(\overline{Q_{1}}+\bar{R}\right) \bmod p^{i} E,
$$

where $\overline{Q_{1}}$ and $\bar{R}$ are the class of $Q_{1}$ and $R$ in the quotient $D_{k} / \varpi^{i} D_{k}$. This proves that the map $\lambda$ is surjective. Suppose now that $\lambda(\bar{P})=0$ for some $P \in D_{k}$, then there exist $Q \in \widehat{D}_{k+1}$ and $R \in D_{k}$ such that $P=\varpi^{i}(Q+R)$. As

$$
\varpi^{i} Q=P-\varpi^{i} R \in D_{k},
$$

we see from the explicit description of $D_{k+1}$ above that $Q \in D_{k+1}$ and finally that $P \in$ $\varpi^{i} D_{k}$, which proves that $\lambda$ is injective and thus an isomorphism. This completes the proof of the auxiliary result.

Now, to prove the proposition, it is enough to prove that $E$ is noetherian. If this is the case, then $\widehat{E}$ is flat over $E$, thus $\widehat{E}_{\mathbb{Q}}$ is flat over $E_{\mathbb{Q}}$ and $\widehat{D}_{k, \mathbb{Q}}$ is flat over $\widehat{D}_{k+1, \mathbb{Q}}$. 
Recall [6, (2.2.5)] that the ring $D_{k}$ is generated by the algebra $B$ and the elements $\varpi^{k p^{a}} \partial_{i}^{\left\langle p^{a}\right\rangle}=\varpi^{k p^{a}} \partial_{i}^{\left[p^{a}\right]}$ with $1 \leqslant i \leqslant M, 1 \leqslant a \leqslant m$. Let us define the following algebras: let $E_{0}=\widehat{D}_{k+1}$, and for $j \geqslant 1 E_{j}$ be the $B$-algebra of $E$ generated by $\widehat{D}_{k+1}$ and the $\varpi^{k \nu_{i}} \partial_{i}^{\left\langle\nu_{i}\right\rangle}$ with $1 \leqslant i \leqslant j$ and $\nu_{i} \in \mathbb{N}$. We also introduce for each $j$ and $s$ an integer satisfying $1 \leqslant s \leqslant m$ the subalgebra $E_{j, s}$ of $E_{j}$ generated by $E_{j-1}$ and the $\varpi^{k p^{a}} \partial_{j}^{\left[p^{a}\right]}$ with $1 \leqslant a \leqslant s$. We define $E_{j, 0}=E_{j-1}$ for $j \geqslant 1$. By definition, we have $E_{j, m}=E_{j}$. Now we use the

Auxiliary result 2.2.18. For each $j \leqslant M, s \leqslant m$, the algebra $E_{j, s}$ is two-sided noetherian. The algebra $E$ is two-sided noetherian.

Proof. We will prove the result by induction on both $j$ and $s$. Note that $E_{0}=\widehat{D}_{k+1}$ is noetherian by 2.2.2. By definition, $E_{0,0}=E_{0}$ and is thus noetherian.

Next, let us prove that if $E_{j, s-1}$ is noetherian, then $E_{j, s}$ is noetherian if $1 \leqslant s \leqslant m$ and $1 \leqslant j \leqslant M$. For this, note that, if $b \in B$, we have as in [6, 3.5.3.2],

$$
\left[\varpi^{k p^{s}} \partial_{j}^{\left[p^{s}\right]}, b\right]=\sum_{i<p^{s}}\left(\begin{array}{c}
p^{s} \\
i
\end{array}\right) \partial_{j}^{\left[p^{s}-i\right]}(b) \varpi^{k p^{s}} \partial_{j}^{[i]},
$$

thus noticing that if $i<p^{s}, \varpi^{k i} \partial_{j}^{[i]} \in E_{j, s-1}$, we have

$$
\left[\varpi^{k p^{s}} \partial_{j}^{\left[p^{s}\right]}, b\right] \in \sum_{i<p^{s}} \hat{D}^{(k+1)} \varpi^{k p^{i}} \partial_{j}^{[i]} \subset E_{j, s-1} .
$$

Consider the finite type $\widehat{D}^{(k+1)}$-module $F:=\sum_{i<p^{s}} \widehat{D}^{(k+1)} \varpi^{k p^{i}} \partial_{j}^{[i]}$. Then for each finite

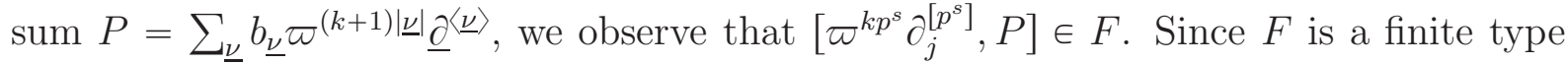
$\widehat{D}^{(k+1)}$-module, it is $p$-adically complete, and thus also for each infinite sum $P \in \widehat{D}^{(k+1)}$, we have $\left[\varpi^{k p^{s}} \partial_{j}^{\left[p^{s}\right]}, P\right] \in F \subset E_{j, s-1}$. Moreover, $\left[\varpi^{k p^{s}} \partial_{j}^{\left[p^{s}\right]}, \varpi^{k p^{a}} \partial_{j}^{\left[p^{a}\right]}\right]=0$ for $a \leqslant s-1$. As $E_{j, s-1}$ is an algebra and as we have the formula for $P, Q \in E_{j}$

$$
\left[\varpi^{k p^{s}} \partial_{j}^{\left[p^{s}\right]}, P Q\right]=\left[\varpi^{k p^{s}} \partial_{j}^{\left[p^{s}\right]}, P\right] Q+P\left[\varpi^{k p^{s}} \partial_{j}^{\left[p^{s}\right]}, Q\right],
$$

we see that $\forall P \in E_{j, s-1}$

$$
\left[\varpi^{k p^{s}} \partial_{j}^{\left[p^{s}\right]}, P\right] \in E_{j, s-1} .
$$

Then, by induction on $l$, we deduce that

$$
\forall l \geqslant 1, \forall P \in E_{j, s-1},\left[\left(\varpi^{k p^{s}} \partial_{j}^{\left[p^{s}\right]}\right)^{l}, P\right] \in \sum_{i \leqslant l-1} E_{j, s-1}\left(\varpi^{k p^{s}} \partial_{j}^{\left[p^{s}\right]}\right)^{i} .
$$

Define $\Delta:=\varpi^{k p^{s}} \partial_{j}^{\left[p^{s}\right]}$ for the rest of the proof. We follow now Berthelot's argument in the proof of [6, 3.5.3]. We do the proof for 'left noetherian' (the right version is similar). Let $I$ be a left ideal of $E_{j, s}$ and $J$ be the set of elements $R$ of $E_{j, s-1}$ such that there exists $P \in I$ that can be written

$$
P=R \Delta^{l}+\sum_{i \leqslant l-1} R_{i} \Delta^{i}
$$


with $R_{i} \in E_{j, s-1}$. If $R_{1}$ and $R_{2}$ are in $J$, write

$$
P_{1}=R_{1} \Delta^{l_{1}}+\sum_{i \leqslant l_{1}-1} R_{i} \Delta^{i}, \text { and } P_{2}=R_{2} \Delta^{l_{2}}+\sum_{i \leqslant l_{2}-1} R_{i}^{\prime} \Delta^{i}
$$

with $R_{i}$ and $R_{i}^{\prime}$ elements of $E_{j, s-1}$. Assume $l_{1} \geqslant l_{2}$, then, using (2.2.19), we can write

$$
P_{1}+\Delta^{l_{1}-l_{2}} P_{2}=\left(R_{1}+R_{2}\right) \Delta^{l_{1}}+\sum_{i \leqslant l_{1}-1} R_{i}^{\prime \prime} \Delta^{i} \in I,
$$

with elements $R_{i}^{\prime \prime} \in E_{j, s-1}$. In particular, we deduce from this that $J$ is a left ideal of $E_{j, s-1}$ generated by a finite set of elements $R_{1}, \ldots, R_{a}$. Moreover $I \bigcap E_{j, s-1}$ is a left ideal of $E_{j, s-1}$ generated by a finite set of elements $Q_{1}, \ldots, Q_{b}$. We see easily then that $I$ is generated by the elements $R_{1}, \ldots, R_{a}, Q_{1}, \ldots, Q_{b}$. This proves that $E_{j, s}$ is noetherian and ends the proof of the auxiliary result.

As we have remarked above, the proof of the proposition 2.2.16 is now complete.

Corollary 2.2.20. Let $\mathfrak{U} \subset \mathfrak{X}$ be an open affine formal subscheme of $\mathfrak{X}, k, k^{\prime} \geqslant k_{\mathfrak{X}}$, such that $k^{\prime} \geqslant k$, then $\Gamma\left(\mathfrak{U}, \widehat{\mathcal{D}}_{\mathfrak{X}, \mathbb{Q}}^{(k, m)}\right)$ is left and right flat over $\Gamma\left(\mathfrak{U}, \widehat{\mathcal{D}}_{\mathfrak{X}, \mathbb{Q}}^{\left(k^{\prime}, m\right)}\right)$.

Proof. Denote $\widehat{D}_{k, \mathbb{Q}}=\Gamma\left(\mathfrak{U}, \widehat{\mathcal{D}}_{\mathfrak{X}, \mathbb{Q}}^{(k, m)}\right)$ and similarly for $k^{\prime}$. Let $M \hookrightarrow N$ be an injection of finite type $\widehat{D}_{k^{\prime}, \mathbb{Q}^{-}}$modules and put

$$
\mathcal{M}=\widehat{\mathcal{D}}_{\mathfrak{U}, \mathbb{Q}}^{\left(k^{\prime}, m\right)} \otimes_{\widehat{D}_{k^{\prime}, \mathbb{Q}}} M, \quad \text { resp. } \mathcal{N}=\widehat{\mathcal{D}}_{\mathfrak{U}, \mathbb{Q}}^{\left(k^{\prime}, m\right)} \otimes_{\widehat{D}_{k^{\prime}, \mathbb{Q}}} N .
$$

Using the equivalence of categories and the exactness in 2.2.6. we have an injection of coherent $\widehat{\mathcal{D}}_{\mathfrak{U}, \mathbb{Q}}^{\left(k^{\prime}, m\right)}$-modules $\mathcal{M} \hookrightarrow \mathcal{N}$. Using the previous flatness result 2.2.16, we have an injection of coherent $\widehat{\mathcal{D}}_{\mathfrak{U}, \mathbb{Q}}^{(k, m)}$-modules

$$
\widehat{\mathcal{D}}_{\mathfrak{U}, \mathbb{Q}}^{(k, m)} \otimes_{\widehat{\mathcal{D}}_{\mathfrak{U}, \mathbb{Q}}^{\left(k^{\prime}, m\right)}} \mathcal{M} \hookrightarrow \widehat{\mathcal{D}}_{\mathfrak{U}, \mathbb{Q}}^{(k, m)} \otimes_{\widehat{\mathcal{D}}_{\mathfrak{U}, \mathbb{Q}}^{\left(k^{\prime}, m\right)}} \mathcal{N}
$$

Taking global sections and using again 2.2 .6 , we observe that

$$
\begin{aligned}
\Gamma\left(\mathfrak{U}, \widehat{\mathcal{D}}_{\mathfrak{U}, \mathbb{Q}}^{(k, m)} \otimes_{\hat{\mathcal{D}}_{\mathfrak{U}, \mathbb{Q}}^{\left(k^{\prime}, m\right)}} \mathcal{M}\right) & \simeq \Gamma\left(\mathfrak{U}, \widehat{\mathcal{D}}_{\mathfrak{U}, \mathbb{Q}}^{(k, m)} \otimes_{\widehat{D}_{k^{\prime}}} M\right) \\
& \simeq \Gamma\left(\mathfrak{U}, \widehat{\mathcal{D}}_{\mathfrak{U}, \mathbb{Q}}^{(k, m)} \otimes_{\hat{D}_{k}} \widehat{D}_{k} \otimes_{\hat{D}_{k^{\prime}}} M\right) \\
& \simeq \widehat{D}_{k, \mathbb{Q}} \otimes_{\hat{D}_{k^{\prime}, \mathbb{Q}}} M
\end{aligned}
$$

and similarly for $\mathcal{N}$. Finally, with formula (2.2.21) we arrive at an injection

$$
\widehat{D}_{k, \mathbb{Q}} \otimes_{\widehat{D}_{k^{\prime}, \mathbb{Q}}} M \hookrightarrow \widehat{D}_{k, \mathbb{Q}} \otimes_{\widehat{D}_{k^{\prime}, \mathbb{Q}}} N,
$$

which proves the corollary.

We compare now rings with different levels $k$. Let $e=e\left(L / \mathbb{Q}_{p}\right)$ be the ramification index of the extension $\mathbb{Q}_{p} \subseteq L$.

Proposition 2.2.22. Let $e^{\prime} \in \mathbb{N}$ such that $e^{\prime} \geqslant \frac{e}{p-1}$. 
(i) If $k \geqslant k_{\mathfrak{X}}$, and $k^{\prime} \geqslant k+e^{\prime}$, then we have the following inclusions of sheaves of rings

$$
\mathcal{D}_{\mathfrak{X}, k^{\prime}}^{\dagger} \hookrightarrow \mathcal{D}_{\mathfrak{X}, k+e^{\prime}}^{\dagger} \hookrightarrow \widehat{\mathcal{D}}_{\mathfrak{X}, \mathbb{Q}}^{(k, 0)} \hookrightarrow \widehat{\mathcal{D}}_{\mathfrak{X}, \mathbb{Q}}^{(k, m)} \hookrightarrow \mathcal{D}_{\mathfrak{X}, k}^{\dagger}
$$

(ii) Suppose $e \leqslant p-1$. If $k \geqslant k_{\mathfrak{X}}$, and $k^{\prime} \geqslant k+1$, then we have the following inclusions of sheaves of rings

$$
\mathcal{D}_{\mathfrak{X}, k^{\prime}}^{\dagger} \hookrightarrow \mathcal{D}_{\mathfrak{X}, k+1}^{\dagger} \hookrightarrow \widehat{\mathcal{D}}_{\mathfrak{X}, \mathbb{Q}}^{(k, 0)} \hookrightarrow \widehat{\mathcal{D}}_{\mathfrak{X}, \mathbb{Q}}^{(k, m)} \hookrightarrow \mathcal{D}_{\mathfrak{X}, k}^{\dagger} .
$$

Proof. It is enough to prove (i). The only non trivial inclusion is

$$
\mathcal{D}_{\mathfrak{X}, k+e^{\prime}}^{\dagger} \hookrightarrow \widehat{\mathcal{D}}_{\mathfrak{X}, \mathbb{Q}}^{(k, 0)},
$$

which we may prove locally over some affine open $\mathfrak{U} \subset \mathfrak{X}$ of the basis of open sets $\mathcal{B}$ from 2.2.2. We use the following notations

$$
B=\Gamma\left(\mathfrak{U}, \mathcal{O}_{\mathfrak{X}}\right), \quad \widehat{D}_{k}=\Gamma\left(\mathfrak{U}, \widehat{\mathcal{D}}_{\mathfrak{X}}^{(k, 0)}\right), D_{k}^{\dagger}=\Gamma\left(\mathfrak{U}, \mathcal{D}_{\mathfrak{X}, k}^{\dagger}\right) .
$$

As before (2.1.14), we have then the following descriptions, assuming that $\operatorname{pr}^{*} \mathcal{T}_{\mathfrak{X}_{0}}$ is free restricted to $\mathfrak{U}$, with basis $\partial_{1}, \ldots, \partial_{M}$,

$$
\begin{gathered}
\widehat{D}_{k}=\left\{\sum_{\underline{\nu}} \varpi^{k|\underline{\nu}|} b_{\underline{\nu}} \underline{\partial}^{\underline{\nu}} \mid b_{\underline{\nu}} \in B, b_{\underline{\nu}} \rightarrow 0\right\}, \\
D_{k}^{\dagger}=\left\{\sum_{\underline{\nu}} \varpi^{k|\underline{\underline{\nu}}|} b_{\underline{\nu}} \underline{\partial}^{[\underline{\nu}]} \mid b_{\underline{\nu}} \in B_{\mathbb{Q}} \text { and } \exists C>0, \eta<1 \mid\left\|b_{\underline{\nu}}\right\|<C \eta^{|\underline{\nu}|}\right\},
\end{gathered}
$$

where $\|\cdot\|$ is any Banach algebra norm on $B_{\mathbb{Q}}$. For the rest of the proof we endow $B_{\mathbb{Q}}$ with the gauge norm $\|\cdot\|$ associated with the lattice $B \subset B_{\mathbb{Q}}$. We need the following Auxiliary result 2.2.23. Let $\underline{\nu}=\left(\nu_{1}, \ldots, \nu_{M}\right)$, then we have

$$
\frac{|\underline{\nu}|}{p-1}-M \log _{p}(|\underline{\nu}|+1)-M \leqslant v_{p}(\underline{\nu} !) \leqslant \frac{|\underline{\nu}|}{p-1} .
$$

Proof. [19, 1.1.2].

Let $P=\sum_{\underline{\nu}} \varpi^{\left(k+e^{\prime}\right)|\underline{\mid}|} b_{\underline{\nu}} \underline{\partial}^{[\underline{\nu}]} \in D_{k+e^{\prime}}^{\dagger}$, then there exist $R>0$ and $S>0$ such that

We can write

$$
\log _{p}\left\|b_{\underline{\nu}}\right\|>R|\underline{\nu}|-S
$$

$$
P=\sum_{\underline{\nu}} \varpi^{k|\underline{\nu}|} c_{\underline{\nu}} \underline{\partial}^{\underline{\nu}} \quad \text { with } c_{\underline{\nu}}=\frac{\varpi^{e^{\prime}|\underline{\nu}|}}{\underline{\nu} !} b_{\underline{\nu}} .
$$

Then the following inequality holds

$$
\log _{p}\left\|c_{\underline{\nu}}\right\|>\left(\frac{e^{\prime}}{e}-\frac{1}{p-1}+R\right)|\underline{\nu}|-S .
$$


Under the conditions of the statement, $\log _{p}\left\|c_{\underline{\nu}}\right\| \rightarrow+\infty$ if $|\underline{\nu}| \rightarrow+\infty$, which proves that $P \in \widehat{D}_{k, \mathbb{Q}}$. This ends the proof of the proposition 2.2 .22

We now complete these results by additional flatness results when $k$ varies.

Proposition 2.2.24. Let $k^{\prime} \geqslant k$.

(i) The morphism of sheaves of rings $\mathcal{D}_{\mathfrak{X}, k^{\prime}}^{\dagger} \hookrightarrow \mathcal{D}_{\mathfrak{X}, k}^{\dagger}$ is left and right flat.

(ii) Let $\mathfrak{U} \subset \mathfrak{X}$ be an open affine. Then $\Gamma\left(\mathfrak{U}, \mathcal{D}_{\mathfrak{X}, k}^{\dagger}\right)$ is left and right flat over $\Gamma\left(\mathfrak{U}, \mathcal{D}_{\mathfrak{X}, k^{\prime}}^{\dagger}\right)$.

Proof. It is enough to prove (ii). Denote $D_{k}^{\dagger}=\Gamma\left(\mathfrak{U}, \mathcal{D}_{\mathfrak{X}, k}^{\dagger}\right)$ (resp. for $k^{\prime}$ ), and for any integer $m \geqslant 0, \widehat{D}_{k, \mathbb{Q}}^{(m)}=\Gamma\left(\mathfrak{U}, \mathcal{D}_{\mathfrak{X}, \mathbb{Q}}^{(k, m)}\right)$ (resp. for $\left.k^{\prime}\right)$. Since $\mathfrak{U}$ is quasi-compact, we know that

$$
D_{k}^{\dagger}=\underset{m}{\lim } \widehat{D}_{k, \mathbb{Q}}^{(m)} .
$$

Let $u: M \hookrightarrow N$ be an injection of coherent $D_{k^{\prime}}^{\dagger}$-modules, then we have the

Auxiliary result 2.2.25. There exist an integer $m \geqslant 0$ and an injection $u^{(m)}$ of coherent $\widehat{D}_{k^{\prime}, \mathbb{Q}^{-}}^{(m)}$ modules $u^{(m)}: M^{(m)} \hookrightarrow N^{(m)}$ such that following properties are satisfied.

(i) There are canonical isomorphisms

$$
D_{k^{\prime}}^{\dagger} \otimes_{\widehat{D}_{k^{\prime}, \mathbb{Q}}^{(m)}} M^{(m)} \stackrel{\alpha}{\simeq} M, \text { and } D_{k^{\prime}}^{\dagger} \otimes_{\widehat{D}_{k^{\prime}, \mathbb{Q}}^{(m)}} N^{(m)} \stackrel{\beta}{\simeq} N
$$

(ii) There is a commutative diagram of coherent $D_{k^{\prime}}^{\dagger}$-modules

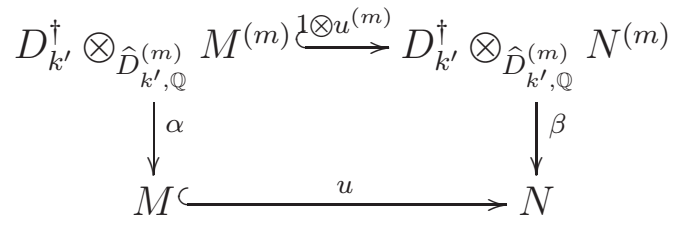

Proof. In order to prove the auxiliary result, we first remark that the morphism $u$ can be extended to any finite presentation of $M$ and $N$ as $D_{k^{\prime}}^{\dagger}$-modules. Thus, there exists a diagram of presentations of $D_{k^{\prime}}^{\dagger}$-modules

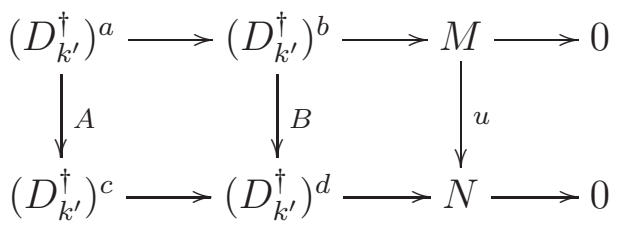

Furthermore, there exists $m$ such that the matrices of the maps $A$ and $B$ have coefficients in $\widehat{D}_{k^{\prime}, \mathbb{Q}}^{(m)}$ and define maps

$$
A:\left(\widehat{D}_{k^{\prime}, \mathbb{Q}}^{(m)}\right)^{a} \rightarrow\left(\widehat{D}_{k^{\prime}, \mathbb{Q}}^{(m)}\right)^{b}(\text { resp. for } B),
$$




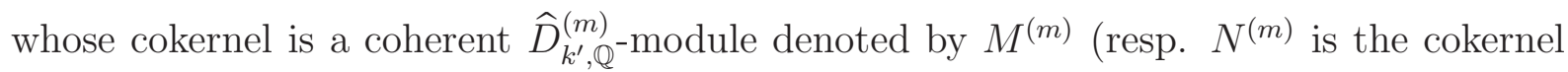
of $B$ ). We finally get from this the following commutative diagram of exact sequences

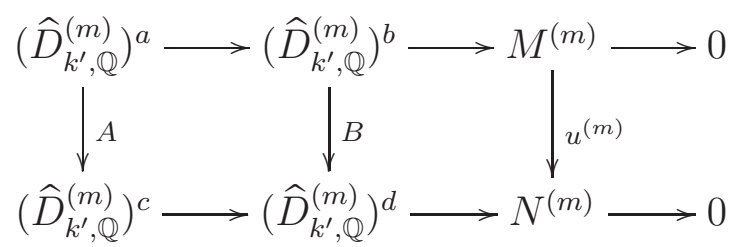

where by definition $u^{(m)}$ is the induced map by $u$ between $M^{(m)}$ and $N^{(m)}$. By construction there are canonical isomorphism

$$
D_{k^{\prime}}^{\dagger} \otimes_{\widehat{D}_{k^{\prime}, \mathbb{Q}}^{(m)}} M^{(m)} \simeq M(\text { resp. for } N) .
$$

Define now $K$ to be the kernel of the map $u^{(m)}: M^{(m)} \rightarrow N^{(m)}$, then as $D_{k^{\prime}}^{\dagger}$ is flat over $\widehat{D}_{k^{\prime}, \mathbb{Q}}^{(m)}$ by 2.2.11, we have an exact sequence of $D_{k^{\prime}}^{\dagger}$-modules

$$
0 \rightarrow D_{k^{\prime}}^{\dagger} \otimes_{\widehat{D}_{k^{\prime}, \mathbb{Q}}^{(m)}} K \rightarrow M \rightarrow N,
$$

showing that

$$
D_{k^{\prime}}^{\dagger} \otimes_{\widehat{D}_{k^{\prime}, \mathbb{Q}}^{(m)}} K=0
$$

and again, as $D_{k^{\prime}}^{\dagger}$ is flat over $\widehat{D}_{k^{\prime}, \mathbb{Q}}^{(m)}$, that

$$
D_{k^{\prime}}^{\dagger} \otimes_{\hat{D}_{k^{\prime}, \mathbb{Q}}^{(m)}}\left(M^{(m)} / K\right) \simeq D_{k^{\prime}}^{\dagger} \otimes_{\widehat{D}_{k^{\prime}, \mathbb{Q}}^{(m)}} M^{(m)} \simeq M
$$

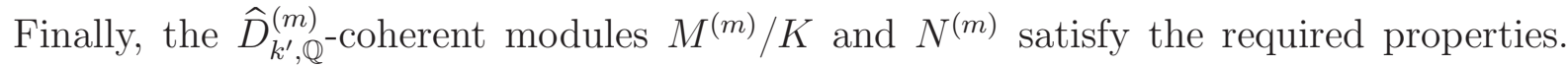
This ends the proof of the auxiliary result.

Take $M^{(m)}, N^{(m)}$ and $u^{(m)}: M^{(m)} \hookrightarrow N^{(m)}$ as given by the auxiliary result. As $\widehat{D}_{k, \mathbb{Q}}^{(m)}$ is flat over $\widehat{D}_{k^{\prime}, \mathbb{Q}}^{(m)}$ by 2.2.20, we have an injection of coherent $\widehat{D}_{k, \mathbb{Q}}^{(m)}$-modules

$$
\widehat{D}_{k, \mathbb{Q}}^{(m)} \otimes_{\widehat{D}_{k^{\prime}, \mathbb{Q}}^{(m)}} M^{(m)} \hookrightarrow \widehat{D}_{k, \mathbb{Q}}^{(m)} \otimes_{\hat{D}_{k^{\prime}, \mathbb{Q}}^{(m)}} N^{(m)} .
$$

We can tensor this map by $D_{k}^{\dagger}$ which is a flat $\widehat{D}_{k, \mathbb{Q}}^{(m)}$-module 2.2.11, and use the properties of $M^{(m)}$ and $N^{(m)}$ to get an injection

$$
D_{k}^{\dagger} \otimes_{D_{k^{\prime}}^{\dagger}} M \hookrightarrow D_{k}^{\dagger} \otimes_{D_{k^{\prime}}^{\dagger}} N
$$

and this proves the proposition.

We end this part by some global properties of coherent sheaves over $\mathfrak{X}$ (resp. coherent $D_{k}^{\dagger}$-modules) when the base $\mathfrak{X}_{0}$ is affine. In general, the formal scheme $\mathfrak{X}$ is projective over $\mathfrak{X}_{0}$ and we can consider the Serre twist $\mathcal{O}_{\mathfrak{X}}(1)$. It is a locally free sheaf of rank 1 over $\mathfrak{X}$. If $\mathfrak{U}_{0}$ is an open affine formal subscheme of $\mathfrak{X}_{0}$, and if $t \in \mathcal{I}\left(\mathfrak{U}_{0}\right)$, then the restriction 
of the sheaf $\mathcal{O}_{\mathfrak{X}}(1)$ to the open subset $D_{+}(t)$ is generated by $t$. As usual, $\mathcal{I}$ denotes the ideal sheaf on $\mathfrak{X}_{0}$ which gives rise to the blow-up $\mathfrak{X}$.

If $\mathcal{M}$ is a sheaf of $\mathcal{O}_{\mathfrak{X}}$-modules over $\mathfrak{X}$ and if $r \in \mathbb{Z}$, then we let $\mathcal{M}(r)$ denote the twisted sheaf

$$
\mathcal{M}(r)=\mathcal{M} \otimes_{\mathfrak{X}} \mathfrak{X}(r) .
$$

Lemma 2.2.26. Let $\mathfrak{X}_{0}$ be a noetherian affine formal scheme. For all integers $r$, there is an isomorphism $\mathcal{O}_{\mathfrak{X}, \mathbb{Q}}(-r) \simeq \mathcal{O}_{\mathfrak{X}, \mathbb{Q}}(-1)$.

Proof. We can assume as in 2.1.3, that $\mathfrak{X}_{0}=\operatorname{Spf}(A)$, and that the ideal $\mathcal{I}$ is generated by the elements $\left(\varpi^{k_{\mathfrak{X}}}, f_{1}, \ldots, f_{r}\right)$. The formal scheme $\mathfrak{X}$ is then covered by the open formal subschemes

$$
D_{+}\left(f_{i}\right)=\operatorname{Spf} A\left\{\frac{f_{j}}{f_{i}}\right\}
$$

and

$$
\mathcal{O}_{\mathfrak{X}}(-1)\left(D_{+}\left(f_{i}\right)\right) \simeq A\left\{\frac{f_{j}}{f_{i}}\right\} \cdot \frac{1}{u_{i}},
$$

where $u_{i}$ is a generator of $\mathcal{O}_{\mathfrak{X}}(1)_{\mid D_{+}\left(f_{i}\right)}$. By definition, we have a section

$$
\alpha=\frac{\varpi^{k_{\mathfrak{X}}}}{u_{0}} \in \mathcal{O}_{\mathfrak{X}}(-1)\left(D_{+}\left(\varpi^{k_{\mathfrak{X}}}\right)\right) .
$$

Moreover the following equations hold

$$
\begin{gathered}
\frac{\varpi^{k_{\mathfrak{X}}}}{u_{0}}=\frac{f_{i}}{u_{i}} \in \mathcal{O}_{\mathfrak{X}}(-1)\left(D_{+}\left(f_{i}\right)\right), \\
\frac{\varpi^{k_{\mathfrak{X}}}}{u_{i}}=\frac{\varpi^{k_{\mathfrak{X}}}}{f_{i}} \cdot \frac{\varpi^{k_{\mathfrak{X}}}}{u_{0}} \in \mathcal{O}_{\mathfrak{X}}\left(D_{+}\left(f_{i}\right)\right) \cdot \frac{\varpi^{k_{\mathfrak{X}}}}{u_{0}} .
\end{gathered}
$$

This proves that $\mathcal{O}_{\mathfrak{X}, \mathbb{Q}}(-1)$ is free with basis $\alpha=\varpi^{k_{\mathfrak{X}}} / u_{0}$. If $r \geqslant 0, \mathcal{O}_{\mathfrak{X}, \mathbb{Q}}(-r) \simeq$ $\mathcal{O}_{\mathfrak{X}, \mathbb{Q}}(-1)^{\otimes r}$ is thus a free $\mathcal{O}_{\mathfrak{X}, \mathbb{Q}}$-module of rank one as well. Since $\mathcal{O}_{\mathfrak{X}, \mathbb{Q}}(r)$ is the dual

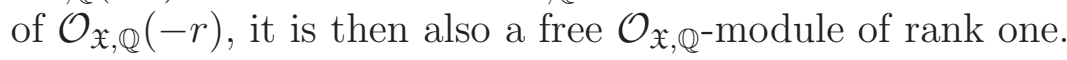

We assume from now on until the rest of this subsection 2.2 that the formal scheme $\mathfrak{X}_{0}$ is affine. Let $k \geqslant k_{\mathfrak{X}}$. To simplify the notation, we write $\mathcal{D}:=\mathcal{D}_{\mathfrak{X}}^{(k, m)}$ and we denote by $\widehat{\mathcal{D}}$ the $p$-adic completion of this sheaf. We also let $\mathcal{D}^{\dagger}=\underline{\lim }_{m} \widehat{\mathcal{D}}_{\mathfrak{X}, \mathbb{Q}}^{(k, m)}$.

Let us first consider the reduction $\mathcal{D}_{i}=\widehat{\mathcal{D}} / \varpi^{i+1} \widehat{\mathcal{D}}$. This is a coherent sheaf thanks to 2.2.7 and a quasi-coherent sheaf of $\mathcal{O}_{X_{i}}$-modules. Let $\mathcal{M}$ be a coherent $\mathcal{D}_{i}$-module.

Lemma 2.2.27. There exist $a, r \in \mathbb{N}$ such that there is a surjection of coherent $\mathcal{D}_{i}$-modules

$$
\left(\mathcal{D}_{i}(-a)\right)^{r} \rightarrow \mathcal{M}
$$


Proof. As the sheaf $\mathcal{M}$ is quasi-coherent over the noetherian scheme $X_{i}$, it is an inductive limit of its $\mathcal{O}_{X_{i}}$-coherent subsheaves. Moreover $\mathcal{D}_{i}$ is an inductive limit of the coherent sheaves $\mathcal{D}_{i, n}$ of differential operators of order less than $n$. We can thus write

$$
\mathcal{M}=\underset{n \in \mathbb{N}}{\lim _{\longrightarrow}} \mathcal{M}^{(n)}
$$

where $\mathcal{M}^{(n)}$ is a coherent $\mathcal{O}_{X_{i}}$-module. Take an open affine subscheme $U \subset X_{i}$. Then $\mathcal{D}_{i}(U)$ is noetherian and $\mathcal{M}(U)$ is a $\mathcal{D}_{i}(U)$-module of finite type. Hence, there exists $N>0$ such that

$$
\underset{n}{\lim _{n}} \mathcal{D}_{i}(U) \cdot \mathcal{M}^{(n)}(U)=\mathcal{D}_{i}(U) \cdot \mathcal{M}^{(N)}(U) \subset \mathcal{M}(U)
$$

Since

$$
\mathcal{M}(U)=\underset{n}{\lim } \mathcal{D}_{i}(U) \cdot \mathcal{M}^{(n)}(U)
$$

we see that $\mathcal{D}_{i}(U) \cdot \mathcal{M}^{(N)}(U)=\mathcal{M}(U)$ and using (iii) of 2.2.7, we find a surjection $\mathcal{D}_{U} \otimes \mathcal{M}_{\mid U}^{(N)} \rightarrow \mathcal{M}_{\mid U}$. As $X_{i}$ is quasi-compact, $X_{i}$ can be covered by a finite number of affine open subsets. There exists therefore $N^{\prime}$ and a surjection $\mathcal{D}_{i} \otimes \mathcal{M}^{\left(N^{\prime}\right)} \rightarrow \mathcal{M}$. As $\mathcal{M}^{\left(N^{\prime}\right)}$ is a coherent $\mathcal{O}_{X_{i}}$-module, there exist $r \in \mathbb{N}, a \in \mathbb{N}$ and a surjection $\left(\mathcal{O}_{X_{i}}(-a)\right)^{r} \rightarrow \mathcal{M}^{\left(N^{\prime}\right)}$ and this proves the lemma.

Lemma 2.2.28. (i) There exists $a \geqslant 0$ such that

$$
\forall b \geqslant a, \forall l>0, H^{l}\left(X_{i}, \mathcal{D}_{i}(b)\right)=0 .
$$

(ii) Let $\mathcal{M}$ be a coherent $\mathcal{D}_{i}$-module, then there exists $a \geqslant 0$ such that

$$
\forall b \geqslant a, \forall l>0, H^{l}\left(X_{i}, \mathcal{M}(b)\right)=0 .
$$

Proof. We have $R \Gamma\left(X_{i}, \mathcal{D}_{i}(b)\right)=R \Gamma\left(X_{0, i}, \cdot\right) \circ R \operatorname{pr}_{*} \mathcal{D}_{i}(b)$. As $X_{0, i}$ is affine, it is enough to prove that $R^{l} \operatorname{pr}_{*} \mathcal{D}_{i}(b)=0$ for $l \geqslant 1$ and for $b \geqslant a$. Take $\mathfrak{U}_{0} \subset \mathfrak{X}_{0}$ affine, endowed with coordinates $x_{1}, \ldots, x_{M}$ and let $\mathfrak{U}=\operatorname{pr}^{-1}\left(\mathfrak{U}_{0}\right)$ and $U=\mathfrak{U} \times \operatorname{Spec}\left(\mathfrak{o} / \varpi^{i+1} \mathfrak{o}\right)$. Denote by $\mathcal{O}_{\mathfrak{U}}(1)$ the restriction to $\mathfrak{U}$ of the Serre twist over $\mathfrak{X}$. Then $\mathcal{D}_{i \mid U}$ is a free $\mathcal{O}_{U}$-module, so that there exists $c$ such that

$$
\forall b \geqslant c, \forall l>0, H^{l}\left(U, \mathcal{D}_{i}(b)\right)=0 .
$$

By taking the maximum $a$ of the constants $c$ for each affine open $\mathfrak{U}_{0}$ of a finite cover of $\mathfrak{X}_{0}$ by such affine subschemes $\mathfrak{U}_{0}$, we get that $R^{l} \operatorname{pr}_{*} \mathcal{D}_{i}(b)=0$ for $l>0$ and $b \geqslant a$ and this proves part (i) of the lemma. For the second assertion, we will prove the following statement by decreasing induction on $K$ :

For all coherent $\mathcal{D}_{i}$-modules $\mathcal{N}$, there exists $a \geqslant 0$, such that

$$
\forall L \geqslant K, \forall b \geqslant a, H^{L}\left(X_{i}, \mathcal{N}(b)\right)=0 .
$$


If $K \geqslant M+1$, the result is clear, since $\mathcal{N}$ is a quasi-coherent sheaf on a scheme of dimension $M$. Suppose now that the result is true for $K \geqslant 2$, and consider a coherent $\mathcal{D}_{i^{-}}$ module $\mathcal{N}$. By the previous lemma, there exist $a$ and $r$ and a exact sequence of coherent $\mathcal{D}_{i}$-modules

$$
0 \rightarrow \mathcal{M} \rightarrow \mathcal{D}_{i}^{r} \rightarrow \mathcal{N}(a) \rightarrow 0
$$

Tensoring this sequence by $\mathcal{O}_{X_{i}}\left(c_{1}+c_{2}\right)$ where $c_{1}, c_{2}$ are non negative integers and looking at the cohomology long exact sequence, we get exact sequences for all $L$

$$
H^{L}\left(X_{i}, \mathcal{D}_{i}^{r}\left(c_{1}+c_{2}\right)\right) \rightarrow H^{L}\left(X_{i}, \mathcal{N}\left(a+c_{1}+c_{2}\right)\right) \rightarrow H^{L+1}\left(X_{i}, \mathcal{M}\left(c_{1}+c_{2}\right)\right) .
$$

By the induction hypothesis, there exists $c_{1}$ such that $\forall L \geqslant K, \forall d \geqslant c_{1}, H^{L}\left(X_{i}, \mathcal{M}(d)\right)=$ 0 , and by (i), there exists $c_{2}$ such that $\forall L \geqslant 1, \forall d \geqslant c_{2}, H^{L}\left(X_{i}, \mathcal{D}_{i}(d)\right)=0$. Finally, if $d \geqslant a+c_{1}+c_{2}$, for every $L \geqslant K-1 \geqslant 1$, we get that $H^{L}\left(X_{i}, \mathcal{N}(d)\right)=0$. This proves (ii) by induction as claimed.

Proposition 2.2.29. (i) For any coherent $\widehat{\mathcal{D}}$-module $\mathcal{M}$, there exist $a, r \in \mathbb{N}$ and a surjection of coherent $\widehat{\mathcal{D}}$-modules

$$
(\widehat{\mathcal{D}}(-a))^{r} \rightarrow \mathcal{M}
$$

(ii) For any coherent $\widehat{\mathcal{D}}_{\mathbb{Q}}$-module $\mathcal{M}$, there exist $r \in \mathbb{N}$ and a surjection of coherent $\widehat{\mathcal{D}}_{\mathbb{Q}}$-modules

$$
\left(\widehat{\mathcal{D}}_{\mathbb{Q}}\right)^{r} \rightarrow \mathcal{M}
$$

(iii) For any coherent $\mathcal{D}^{\dagger}$-module $\mathcal{M}$, there exist $r \in \mathbb{N}$ and a surjection of coherent $\mathcal{D}^{\dagger}$-modules

$$
\left(\mathcal{D}^{\dagger}\right)^{r} \rightarrow \mathcal{M}
$$

Proof. Let $\mathcal{M}$ be a coherent $\widehat{\mathcal{D}}$-module. For part (i), we need to show that there exists a non negative integer $a$ such that the twist $\mathcal{M}(a)$ is generated by a finite number of global sections. Let $\mathcal{M}_{t}$ be the torsion part of $\mathcal{M}$, which is a coherent submodule of $\mathcal{M}$, since $\widehat{\mathcal{D}}(\mathfrak{U})$ is noetherian for every affine open $\mathfrak{U}$. Over an affine open $\mathfrak{U}$ of $\mathfrak{X}$, the module $\mathcal{M}_{t}(\mathfrak{U})$ is a finite type module over $\widehat{\mathcal{D}}(\mathfrak{U})$ and there exists a constant $c$ such that $\varpi^{c} \mathcal{M}_{t}(\mathfrak{U})=0$. Since $\mathfrak{X}$ can be covered by a finite number of open affine formal subschemes, there exists $L$ such that $\varpi^{L} \mathcal{M}_{t}=0$. Then for $i \geqslant L$, and denoting $\mathcal{G}_{0}=\mathcal{M} /\left(\varpi \mathcal{M}+\mathcal{M}_{t}\right)$ we have exact sequences

$$
0 \rightarrow \mathcal{G}_{0} \stackrel{\varpi^{i}}{\rightarrow} \mathcal{M} / \varpi^{i+1} \mathcal{M} \rightarrow \mathcal{M} / \varpi^{i} \mathcal{M} \rightarrow 0
$$

Since $\mathcal{G}_{0}$ is a coherent $\mathcal{D}_{0}$-module, there exists $a_{1} \geqslant 0$ so that $H^{1}\left(X_{0}, \mathcal{G}_{0}(b)\right)=0$ for every $b \geqslant a_{1}$. As a consequence, if $b \geqslant a_{1}$, for all $i \geqslant L$ we have surjections

$$
\Gamma\left(\mathfrak{X}, \mathcal{M} / \varpi^{i+1} \mathcal{M}(b)\right) \rightarrow \Gamma\left(\mathfrak{X}, \mathcal{M} / \varpi^{i} \mathcal{M}(b)\right) .
$$

Since $\mathcal{M} / \varpi^{L} \mathcal{M}$ is a coherent $\mathcal{D}_{L-1}$-module, there exists $a \geqslant a_{1}$ and a surjection $s$ : $\mathcal{D}_{L-1}^{r} \rightarrow \mathcal{M} / \varpi^{L} \mathcal{M}(a)$ defined by global sections $e_{1}, \ldots, e_{r} \in \Gamma\left(X_{L-1}, \mathcal{M} / \varpi^{L} \mathcal{M}(a)\right)$. Finally we see by induction on $i$ that these sections $e_{1}, \ldots, e_{r}$ can be lifted to global sections 
of $\Gamma\left(\mathfrak{X}, \mathcal{M} / \varpi^{i} \mathcal{M}(a)\right)$ for every $i$, and thus to global sections of $\Gamma(\mathfrak{X}, \mathcal{M}(a))$. These sections define a map $\widehat{\mathcal{D}}^{r} \rightarrow \mathcal{M}(a)$ that is surjective since it is surjective mod $\varpi$. This proves the part (i).

Assertion (ii) follows from (i) and lemma 2.2.26. For (iii), we remark that, if $\mathcal{M}$ is a coherent $\mathcal{D}^{\dagger}$-module, there exists a coherent $\widehat{\mathcal{D}}$-module $\mathcal{N}$ such that

$$
\mathcal{M} \simeq \mathcal{D}^{\dagger} \otimes_{\widehat{\mathcal{D}}} \mathcal{N}
$$

We can then apply (ii) to $\mathcal{N}$ and this proves (iii).

2.3. An invariance theorem for admissible blow-ups. We keep here the hypothesis from the previous section. In particular, $\mathfrak{X}_{0}$ denotes a smooth formal $\mathfrak{S}$-scheme and

$$
\text { pr }: \mathfrak{X} \rightarrow \mathfrak{X}_{0}
$$

denotes an admissible blow-up. Let $\mathrm{pr}^{\prime}: \mathfrak{X}^{\prime} \rightarrow \mathfrak{X}_{0}$ be another admissible formal blowup and let $\pi: \mathfrak{X}^{\prime} \rightarrow \mathfrak{X}$ be a morphism over $\mathfrak{X}_{0}$, inducing an isomorphism between the associated rigid-analytic spaces $\mathfrak{X}_{\mathbb{Q}}$ and $\mathfrak{X}_{\mathbb{Q}}^{\prime}$ (which are both canonically isomorphic to the rigid-analytic space $\mathfrak{X}_{0, \mathbb{Q}}$ associated to $\left.\mathfrak{X}_{0}\right)$. Then we have the following invariance property. This does not make use of the smoothness assumption for $\mathfrak{X}_{0}$.

Proposition 2.3.1. The functors $\pi_{*}$ (resp. $\pi^{*}$ ) are exact on the category of coherent $\mathcal{O}_{\mathfrak{X}^{\prime}, \mathbb{Q}}$-modules (resp. coherent $\mathcal{O}_{\mathfrak{X}, \mathbb{Q}}$-modules) and induce an equivalence of categories between coherent $\mathcal{O}_{\mathfrak{X}^{\prime}, \mathbb{Q}}$-modules and coherent $\mathcal{O}_{\mathfrak{X}, \mathbb{Q}}$-modules.

Proof. Let sp (resp. sp') be the specialization map $\mathfrak{X}_{\mathbb{Q}} \rightarrow \mathfrak{X}\left(\right.$ resp. $\mathfrak{X}_{\mathbb{Q}}^{\prime} \rightarrow \mathfrak{X}^{\prime}$ ). Then by Tate's acyclicity theorem one knows that $\mathrm{sp}_{*}$ is exact over the category of coherent $\mathcal{O}_{\mathfrak{X}_{\mathbb{Q}}}$-modules. Moreover, via specialization, the category of coherent $\mathcal{O}_{\mathfrak{X}_{\mathbb{Q}}}$-modules over the rigid space $\mathfrak{X}_{\mathbb{Q}}$ is equivalent to the category of coherent $\mathcal{O}_{\mathfrak{X}, \mathbb{Q}}$-modules over the formal scheme $\mathfrak{X}\left[\underline{6}\right.$, discussion after (4.1.3.1)] and similarly for $\mathfrak{X}^{\prime}$. Let $\tilde{\pi}$ be the induced map by $\pi$ between the analytic spaces $\mathfrak{X}_{\mathbb{Q}}^{\prime}$ and $\mathfrak{X}_{\mathbb{Q}}$, which is an isomorphism by assumption. One has the following commutative diagram

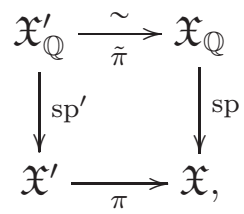

from which we can deduce the following

Lemma 2.3.2. With the previous notations, there is an isomorphism

$$
\mathcal{O}_{\mathfrak{X}, \mathbb{Q}} \simeq \pi_{*} \mathcal{O}_{\mathfrak{X}^{\prime}, \mathbb{Q}} .
$$

Proof. Let us first note that $R \tilde{\pi}_{*} \mathcal{O}_{\mathfrak{X}_{\mathbb{Q}}^{\prime}} \simeq \mathcal{O}_{\mathfrak{X}_{\mathbb{Q}}}$ because $\tilde{\pi}$ is an isomorphism of analytic spaces. Since $R \operatorname{sp}_{*}^{\prime} \mathcal{O}_{\mathfrak{X}^{\prime} \mathbb{Q}}=\mathcal{O}_{\mathfrak{X}^{\prime}, \mathbb{Q}}$ (and the same with $\mathfrak{X}$ ), we can compute using the previous diagram $R \pi_{*} \mathcal{O}_{\mathfrak{X}^{\prime}, \mathbb{Q}} \simeq R \operatorname{sp}_{*} R \tilde{\pi}_{*} \mathcal{O}_{\mathfrak{X}^{\prime} \mathbb{Q}} \simeq \mathcal{O}_{\mathfrak{X}, \mathbb{Q}}$. This proves the lemma. 


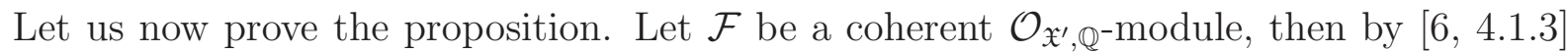
there exists a coherent $\mathcal{O}_{\mathfrak{X}^{\prime} \mathbb{Q}}$-module $\tilde{\mathcal{F}}$ such that $\mathcal{F}=\operatorname{sp}_{*}^{\prime} \tilde{\mathcal{F}}$. Considering again the previous diagram, we compute

$$
R \pi_{*} \mathcal{F} \simeq R \operatorname{sp}_{*} R \tilde{\pi}_{*} \tilde{\mathcal{F}}
$$

As $\tilde{\pi}$ is an isomorphism, $R^{i} \tilde{\pi}_{*} \tilde{\mathcal{F}}=0$ if $i \geqslant 1$ and $\tilde{\pi}_{*} \tilde{\mathcal{F}}$ is a coherent $\mathcal{O}_{\mathfrak{X}_{\mathbb{Q}}}$-module. Finally, $R \mathrm{sp}_{*}$ is reduced to $\mathrm{sp}_{*}$ and the spectral sequence of the composite functors degenerates, giving us that $R^{i} \pi_{*} \mathcal{F}=0$ if $i \geqslant 1$ and $\pi_{*} \mathcal{F}$ is a coherent $\mathcal{O}_{\mathfrak{X}, \mathbb{Q}}$-module. It is moreover clear that $\pi^{*}$ preserves coherence. Consider the map of coherent $\mathcal{O}_{\mathfrak{X}^{\prime}, \mathbb{Q}^{-} \text {modules }} \pi^{*} \pi_{*} \mathcal{F} \rightarrow \mathcal{F}$. To prove that this is an isomorphism is local on $\mathfrak{X}$, which we can assume to be affine. In this case, it is enough to prove the statement for $\mathcal{F}=\mathcal{O}_{\mathfrak{X}^{\prime}, \mathbb{Q}}$, since $\pi_{*}$ is exact. But $\pi^{*} \pi_{*} \mathcal{O}_{\mathfrak{X}^{\prime}, \mathbb{Q}} \simeq \pi^{*} \mathcal{O}_{\mathfrak{X}, \mathbb{Q}}$ because of the lemma and thus $\pi^{*} \pi_{*} \mathcal{O}_{\mathfrak{X}^{\prime}, \mathbb{Q}} \simeq \mathcal{O}_{\mathfrak{X}^{\prime}, \mathbb{Q}}$.

Let $\mathcal{E}$ be a coherent $\mathcal{O}_{\mathfrak{X}, \mathbb{Q}^{-m o d u l e}}$ and consider the canonical map $\mathcal{E} \rightarrow \pi_{*} \pi^{*} \mathcal{E}$. Again, since $\pi_{*}$ is exact, we are reduced to the case where $\mathfrak{X}$ is affine and $\mathcal{E}=\mathcal{O}_{\mathfrak{X}, \mathbb{Q}}$ to prove that this map is an isomorphism. In this case, the isomorphism follows again from the lemma. Since $\pi_{*}$ and $\pi^{*}$ are quasi-inverse to each other, and $\pi_{*}$ is an exact functor, $\pi^{*}$ is exact as well. This finishes the proof of the proposition.

In the sequel, we will give a version of the invariance property 2.3.1 for $\mathcal{D}$-modules. Recall that we have the sheaves of differential operators $\mathcal{D}_{\mathfrak{X}, k}^{\dagger}, \widehat{\mathcal{D}}_{\mathfrak{X}, \mathbb{Q}}^{(k, m)}$ etc. for $k \geqslant k_{\mathfrak{X}}$, cf. previous subsection, at our disposal (similarly for $\mathfrak{X}^{\prime}$ ). In the following we fix a congruence level $k \geqslant \max \left\{k_{\mathfrak{X}}, k_{\mathfrak{X}^{\prime}}\right\}$.

For a $\mathcal{D}_{\mathfrak{X}^{\prime}, k}^{\dagger}$-module $\mathcal{M}$, we let, as usual, $\pi_{*} \mathcal{M}$ denote the push-forward of $\mathcal{M}$ in the sense of abelian sheaves (and analogously in the case of $\widehat{\mathcal{D}}_{\mathfrak{X}^{\prime}, \mathbb{Q}}^{(k, m)}$-modules). Conversely, there is a functor $\pi^{!}$in the other direction constructed as usual using the formalism of inverse images of $\mathcal{D}$-modules: first of all, by definition of the sheaves $\mathcal{D}_{X_{i}^{\prime}}^{(k, m)}$ and $\mathcal{D}_{X_{i}}^{(k, m)}$, cf. (2.1.13), and the fact that $\left(\mathrm{pr}^{\prime}\right)^{*}=\pi^{*} \circ \mathrm{pr}^{*}$ we have

$$
\mathcal{D}_{X_{i}^{\prime}}^{(k, m)} \simeq \pi_{i}^{*} \mathcal{D}_{X_{i}}^{(k, m)}
$$

and the sheaf $\mathcal{D}_{X_{i}^{\prime}}^{(k, m)}$ can be uniquely endowed with a structure of right $\pi^{-1} \mathcal{D}_{X_{i}}^{(k, m)}$-module. Passing to the $p$-adic completion, we see that the sheaf $\widehat{\mathcal{D}}_{\mathfrak{X}^{\prime}}^{(k, m)}$ is a sheaf of right $\pi^{-1} \widehat{\mathcal{D}}_{\mathfrak{X}}^{(k, m)}$ modules. Then, passing to the inductive limit over $m$ implies that $\mathcal{D}_{\mathfrak{X}^{\prime}, k}^{\dagger}$ is a right $\pi^{-1} \mathcal{D}_{\mathfrak{X}, k}^{\dagger}$ module. For a $\mathcal{D}_{\mathfrak{X}, k}^{\dagger}$-module $\mathcal{M}$, we then define

$$
\pi^{!} \mathcal{M}:=\mathcal{D}_{\mathfrak{X}^{\prime}, k}^{\dagger} \otimes_{\pi^{-1} \mathcal{D}_{\mathfrak{X}, k}^{\dagger}} \pi^{-1} \mathcal{M},
$$

and we make the analogous definition in the case of $\widehat{\mathcal{D}}_{\mathfrak{X}, \mathbb{Q}}^{(k, m)}$-modules 2

\footnotetext{
${ }^{2}$ Since $\mathcal{D}_{\mathfrak{X}^{\prime}}^{(k, m)}=\pi^{*} \mathcal{D}_{\mathfrak{X}}^{(k, m)}$, the functor $\pi^{!}$is a version of the usual $D$-module pullback functor 15], whence our notation.
} 
Before stating the next theorem, we need the following lemmas. Denote by $\mathcal{A}$ the abelian category of projective systems $K_{\bullet}=\left(K_{i}\right)_{i \in \mathbb{N}}=\left(K_{0} \leftarrow K_{1} \leftarrow \ldots\right)$ of $\mathcal{O}_{\mathfrak{X}}$-modules $K_{i}$, where $K_{i}$ is annihilated by multiplication by $\varpi^{i+1}$ for every $i \geqslant 0$. Put $\mathcal{P}^{b}=D^{b}(\mathcal{A})$. Note that for a complex $\left(K^{n}\right)_{n \in \mathbb{Z}}$ in $\mathcal{P}^{b}$, where each $K^{n}=\left(K_{i}^{n}\right)_{i \in \mathbb{N}}$, there exists $J>0$ so that $\mathcal{H}^{n}\left(K^{\bullet}\right)=0$ if $|n|>J(n \in \mathbb{Z})$. The functor $\lim _{\longleftarrow}: \mathcal{A} \rightarrow \operatorname{Mod}\left(\mathcal{O}_{\mathfrak{X}}\right)$ extends to a derived functor $R$ lim from the derived category $\mathcal{P}^{b}$ to the bounded derived category

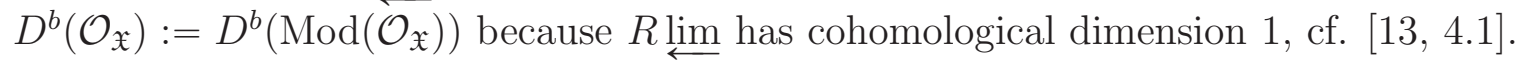

Lemma 2.3.4. Let $N \in \mathbb{N}$, and let $K_{\bullet}$ be an object of $\mathcal{A}$ such that $\varpi^{N} K_{\bullet}=0$, then the complex $\mathbb{Q} \otimes_{\mathbb{Z}} R \lim _{\longleftarrow} K_{\bullet}$ is quasi-isomorphic to 0 in $D^{b}\left(\mathcal{O}_{\mathfrak{X}, \mathbb{Q}}\right)$.

Proof. In the following we consider $K$ • as an object in $\mathcal{P}^{b}$ concentrated in degree zero. By hypothesis, the map $\varpi^{N} \cdot: K_{\bullet} \rightarrow K$ • factorizes through the zero complex

$$
K_{\bullet} \rightarrow 0 \rightarrow K_{\bullet} \text {. }
$$

After applying $R^{k} \lim _{\longleftarrow}=\mathcal{H}^{k} \circ R \lim$, for $k \in \mathbb{Z}$, we find that multiplication with $\varpi^{N}$ factorizes

$$
R^{k} \lim _{\longleftarrow} K_{\bullet} \rightarrow 0 \rightarrow R^{k} \lim _{\longleftarrow} K_{\bullet},
$$

meaning that for every $k \in \mathbb{Z}, \varpi^{N} R^{k} \lim _{\longleftarrow} K_{\bullet}=0$ and thus

$$
\mathbb{Q} \otimes_{\mathbb{Z}} R^{k} \lim _{\bullet} K_{\bullet}=0 .
$$

This proves the lemma, as $\mathbb{Q}$ is flat over $\mathbb{Z}$ and as this module is the $k$-th cohomology sheaf of the complex

$$
\mathbb{Q} \otimes_{\mathbb{Z}} R \lim _{\longleftarrow} K
$$

Lemma 2.3.5. Let $N \in \mathbb{N}$, and let $\mathcal{E}^{\bullet}, \mathcal{F}^{\bullet}$ two objects of $\mathcal{P}^{b}$, and $h: \mathcal{E}^{\bullet} \rightarrow \mathcal{F}^{\bullet}$ a morphism in $\mathcal{P}^{b}$ so that the mapping cone $\mathcal{C}^{\bullet}$ of $h$ (defined up to a quasi-isomorphism) satisfies

$$
\forall j \in \mathbb{Z}: \varpi^{N} \cdot \mathcal{H}^{j}\left(\mathcal{C}^{\bullet}\right)=0
$$

Then the map $h$ induces a quasi-isomorphism

$$
\mathbb{Q} \otimes_{\mathbb{Z}} R \lim _{\longleftarrow}\left(\mathcal{E}^{\bullet}\right) \simeq \mathbb{Q} \otimes_{\mathbb{Z}} R \lim _{\longleftarrow}\left(\mathcal{F}^{\bullet}\right) .
$$

Proof. First note that since $\mathcal{E}^{\bullet}$ and $\mathcal{F}^{\bullet}$ have bounded cohomology, this is also the case of $\mathcal{C}^{\bullet}$, so that the condition of the lemma involves only a finite number of $j \in \mathbb{Z}$. More precisely, there exists $J \in \mathbb{N}$, such that the sheaves $\mathcal{H}^{j}\left(\mathcal{C}^{\bullet}\right)$ are zero for all $j$ satisfying the condition $|j|>J$. Using the cohomological truncations functors $\sigma_{\geqslant n}$ as defined in [14, I, 7], and denoting $\sigma_{>n}$ of loc. cit. by $\sigma_{\geqslant n+1}$, we have for each $n$ a triangle in $\mathcal{P}^{b}$ ([14, I, $7.2])$

$$
\mathcal{H}^{n}\left(\mathcal{C}^{\bullet}\right) \rightarrow \sigma_{\geqslant n}\left(\mathcal{C}^{\bullet}\right) \rightarrow \sigma_{\geqslant n+1}\left(\mathcal{C}^{\bullet}\right) \stackrel{+1}{\longrightarrow}
$$


We will prove by decreasing induction on $n$ that $\mathbb{Q} \otimes R \lim _{\geqslant n}\left(\mathcal{C}^{\bullet}\right)$ is quasi-isomorphic to 0 . This is true if $n=J+1$. Assume that this is true for $n+1$, then after applying $\mathbb{Q} \otimes R \varliminf_{\longleftarrow}$ to the previous triangle, we get a triangle

$$
\mathbb{Q} \otimes R \lim _{\longleftarrow} \mathcal{H}^{n}\left(\mathcal{C}^{\bullet}\right) \rightarrow \mathbb{Q} \otimes R \lim _{\longleftarrow} \sigma_{\geqslant n}\left(\mathcal{C}^{\bullet}\right) \rightarrow \mathbb{Q} \otimes R \lim _{\longleftarrow} \sigma_{\geqslant n+1}\left(\mathcal{C}^{\bullet}\right) \stackrel{+1}{\longrightarrow} .
$$

As by hypothesis $\varpi^{N} \mathcal{H}^{n}\left(\mathcal{C}^{\bullet}\right)=0$, we see by applying the previous lemma 2.3.4 to the projective system $\mathcal{H}^{n}\left(\mathcal{C}^{\bullet}\right)$ that $\mathbb{Q} \otimes R \lim \mathcal{H}^{n}\left(\mathcal{C}^{\bullet}\right)$ is quasi-isomorphic to 0 . Therefore, we have a quasi-isomorphism

$$
\mathbb{Q} \otimes R \lim \sigma_{\geqslant n}\left(\mathcal{C}^{\bullet}\right) \simeq \mathbb{Q} \otimes R \lim \sigma_{\geqslant n+1}\left(\mathcal{C}^{\bullet}\right),
$$

and the complex $\mathbb{Q} \otimes R \lim _{\geqslant n}\left(\mathcal{C}^{\bullet}\right)$ is hence quasi-isomorphic to 0 . Thus, we conclude that for all $n, \mathbb{Q} \otimes R \lim \sigma_{\geqslant n}\left(\mathcal{C}^{\bullet}\right)$ is quasi-isomorphic to 0 . Since $\mathcal{C}^{\bullet}$ has bounded cohomology, it is quasi-isomorphic to $\sigma_{\geqslant n}\left(\mathcal{C}^{\bullet}\right)$ for $n$ small enough and this finally proves that $\mathbb{Q} \otimes R \lim \mathcal{C}^{\bullet}$ is quasi-isomorphic to 0 . Now we consider the triangle in $\mathcal{P}^{b}$

$$
\mathcal{E}^{\bullet} \rightarrow \mathcal{F}^{\bullet} \rightarrow \mathcal{C}^{\bullet} \stackrel{+1}{\rightarrow}
$$

and apply $\mathbb{Q} \otimes R l i m$. In this way we obtain a triangle

$$
\mathbb{Q} \otimes R \lim _{\longleftarrow} \mathcal{E}^{\bullet} \rightarrow \mathbb{Q} \otimes R \lim _{\longleftarrow} \mathcal{F}^{\bullet} \rightarrow \mathbb{Q} \otimes R \lim _{\longleftarrow} \mathcal{C}^{\bullet+1} \rightarrow,
$$

and since $\mathbb{Q} \otimes R \lim _{\longleftarrow} \mathcal{C}^{\bullet}$ is quasi-isomorphic to 0 , we see that the first map of the latter triangle is a quasi-isomorphism as claimed.

As before, we denote the associated rigid analytic space of $\mathfrak{X}$ by $\mathfrak{X}_{\mathbb{Q}}$. From 2.3.1 and the lemma $2.3 .2 R^{j} \pi_{*} \mathcal{O}_{\mathfrak{X}^{\prime}, \mathbb{Q}}=0$ for $j>0$ and $\pi_{*} \mathcal{O}_{\mathfrak{X}^{\prime}, \mathbb{Q}}=\mathcal{O}_{\mathfrak{X}, \mathbb{Q}}$. As the map $\pi$ is proper, the

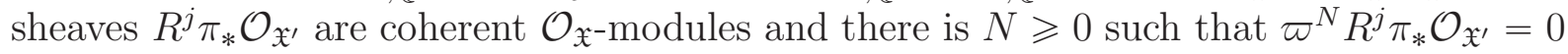
for all $j>0$ and such that the kernel and cokernel of the natural map $\mathcal{O}_{\mathfrak{X}} \rightarrow \pi_{*} \mathcal{O}_{\mathfrak{X}^{\prime}}$ are killed by $\varpi^{N}$ as well. For any $i \geqslant 0$, let as usual $X_{i}$ be the reduction of $\mathfrak{X} \bmod \varpi^{i+1}$ and similarly for $\mathfrak{X}^{\prime}$ and denote by $\pi_{i}: X_{i}^{\prime} \rightarrow X_{i}$ the morphism induced by $\pi$. We will need the following

Auxiliary result 2.3.6. Let $i \geqslant 0$.

(i) Kernel and cokernel of the canonical map $\mathcal{O}_{X_{i}} \rightarrow \pi_{i *} \mathcal{O}_{X_{i}^{\prime}}$ are annihilated by $\varpi^{2 N}$.

(ii) For all $j \geqslant 1$, one has $\varpi^{2 N} R^{j} \pi_{i *} \mathcal{O}_{X_{i}^{\prime}}=0$.

Proof. As the formal scheme $\mathfrak{X}^{\prime}$ is flat, there are exact sequences

$$
0 \longrightarrow \mathcal{O}_{\mathfrak{X}^{\prime}} \stackrel{\varpi^{i+1}}{\longrightarrow} \mathcal{O}_{\mathfrak{X}^{\prime}} \longrightarrow \mathcal{O}_{X_{i}^{\prime}} \longrightarrow 0
$$

Applying $R^{j} \pi_{*}$, we get exact sequences for any $i$ and $j \geqslant 1$,

$$
R^{j} \pi_{*} \mathcal{O}_{\mathfrak{X}^{\prime}} \rightarrow R^{j} \pi_{i *} \mathcal{O}_{X_{i}^{\prime}} \rightarrow R^{j+1} \pi_{*} \mathcal{O}_{\mathfrak{X}^{\prime}}
$$


that prove that $\varpi^{2 N} R^{j} \pi_{i *} \mathcal{O}_{X_{i}^{\prime}}=0$. Moreover we can consider the following commutative diagram of exact sequences

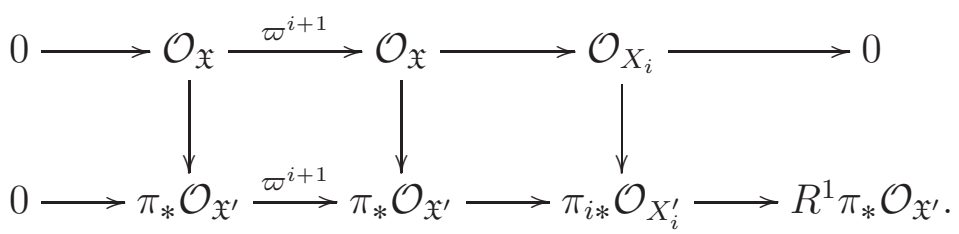

By the snake lemma the kernel of the canonical map $\mathcal{O}_{X_{i}} \rightarrow \pi_{i *} \mathcal{O}_{X_{i}^{\prime}}$ is killed by $\varpi^{2 N}$. By chasing the diagram we also see that the cokernel of this map is also killed by $\varpi^{2 N}$ for all $i$. This proves the auxiliary result.

Lemma 2.3.7. Let $\pi: \mathfrak{X}^{\prime} \rightarrow \mathfrak{X}$ be a morphism over $\mathfrak{X}_{0}$ between admissible formal blow-ups of the smooth formal scheme $\mathfrak{X}_{0}$. Let $k \geqslant \max \left\{k_{\mathfrak{X}}, k_{\mathfrak{X}^{\prime}}\right\}$.

(i) Then we have: $R^{j} \pi_{*} \mathcal{D}_{\mathfrak{X}^{\prime}, k}^{\dagger}=0$ for $j>0$. Moreover, $\pi_{*} \mathcal{D}_{\mathfrak{X}^{\prime}, k}^{\dagger}=\mathcal{D}_{\mathfrak{X}, k}^{\dagger}$.

(ii) There is a canonical isomorphism $\mathcal{D}_{\mathfrak{X}^{\prime}, k}^{\dagger} \simeq \pi^{!} \cdot \mathcal{D}_{\mathfrak{X}, k}^{\dagger}$.

Proof. Since $R^{j} \pi_{*}$ commutes with inductive limits, it suffices to prove the claim for $\widehat{\mathcal{D}}_{\mathfrak{X}^{\prime}, \mathbb{Q}}^{(k, m)}$. Abbreviate $\widehat{\mathcal{D}}_{\mathfrak{X}^{\prime}}=\widehat{\mathcal{D}}_{\mathfrak{X}^{\prime}}^{(k, m)}$ (and similarly for $\mathfrak{X}$ ), and $\mathcal{D}_{X_{i}^{\prime}}=\widehat{\mathcal{D}}_{\mathfrak{X}^{\prime}, k}^{(m)} / \varpi^{i+1} \widehat{\mathcal{D}}_{\mathfrak{X}^{\prime}, k}^{(m)}$ (and similarly for $X_{i}$ ). We need to compute $R \pi_{*} \widehat{\mathcal{D}}_{\mathfrak{X}^{\prime}}$. Note that by [25, Lemma 20.32.4] $R \lim _{i} \mathcal{D}_{X_{i}^{\prime}} \simeq$ $\lim _{i} \mathcal{D}_{X_{i}^{\prime}}$, so that

$$
R \pi_{*} \widehat{\mathcal{D}}_{\mathfrak{X}^{\prime}} \simeq R \pi_{*} R{\underset{i}{\lim }}_{\mathcal{D}_{X_{i}^{\prime}}} \simeq R{\underset{i}{\lim }}_{\lim _{i}} R \pi_{i *} \mathcal{D}_{X_{i}^{\prime}}
$$

by [25, Lemma 20.32.2]. As the sheaf $\mathcal{D}_{X_{i}}$ is a flat $\mathcal{O}_{X_{i}}$-module, the projection formula gives a canonical isomorphism

$$
R \pi_{i *} \mathcal{D}_{X_{i}^{\prime}} \simeq R \pi_{i *} \mathcal{O}_{X_{i}^{\prime}} \otimes_{\mathcal{O}_{X_{i}}} \mathcal{D}_{X_{i}}
$$

so that the canonical map $\mathcal{O}_{X_{i}} \rightarrow R \pi_{i *} \mathcal{O}_{X_{i}^{\prime}}$ induces a map of projective systems of complexes $h:\left(\mathcal{D}_{X_{i}}\right)_{i} \rightarrow\left(R \pi_{i *} \mathcal{D}_{X_{i}^{\prime}}\right)_{i}$. We consider these projective systems as objects of $\mathcal{P}^{b}$. By applying $R \varliminf_{i}$ to $h$, we get the canonical map $\widehat{h}: \widehat{\mathcal{D}}_{\mathfrak{X}} \rightarrow R \pi_{*} \widehat{\mathcal{D}}_{\mathfrak{X}^{\prime}}$. Moreover, we have

$$
\forall j \geqslant 0 \forall i \quad R^{j} \pi_{i *} \mathcal{D}_{X_{i}^{\prime}} \simeq R^{j} \pi_{i *} \mathcal{O}_{X_{i}^{\prime}} \otimes_{\mathcal{O}_{X_{i}}} \mathcal{D}_{X_{i}}
$$

By flat base change from $\mathcal{O}_{X_{i}}$ to $\mathcal{D}_{X_{i}}$, the previous auxiliary result 2.3.6 (i) implies that the kernel and the cokernel of the map $\left(\mathcal{D}_{X_{i}}\right)_{i} \rightarrow\left(\pi_{i *} \mathcal{D}_{X_{i}^{\prime}}\right)_{i}$ of projective systems are annihilated by $\varpi^{2 N}$. Similarly, by 2.3.6 (ii) the projective systems $\left(R^{j} \pi_{i *} \mathcal{D}_{X_{i}^{\prime}}\right)_{i}$ for $j \geqslant 1$ are annihilated by $\varpi^{2 N}$. Let $\mathcal{C} \bullet$ be the cone of $h$, then, as the functor $\mathcal{H}^{0}$ is a cohomological functor [14, definition, p.27] we have the following exact cohomology sequence of projective systems of sheaves

$$
0 \rightarrow\left(\mathcal{H}^{-1}\left(\mathcal{C}^{\bullet}\right)\right) \rightarrow\left(\mathcal{D}_{X_{i}}\right)_{i} \rightarrow\left(\pi_{i *} \mathcal{D}_{X_{i}^{\prime}}\right)_{i} \rightarrow\left(\mathcal{H}^{0}\left(\mathcal{C}^{\bullet}\right)\right) \rightarrow 0
$$


and $\forall j \geqslant 1$

$$
\left(R^{j} \pi_{i *}\left(\mathcal{D}_{X_{i}}\right)\right)_{i} \simeq \mathcal{H}^{j}\left(\mathcal{C}^{\bullet}\right)
$$

We thus see that the cohomology of $\mathcal{C}^{\bullet}$ is annihilated by $\varpi^{2 N}$, so that we can apply lemma 2.3 .5 and obtain a quasi-isomorphism $\widehat{h} \otimes \mathbb{Q}: \widehat{\mathcal{D}}_{\mathfrak{X}, \mathbb{Q}} \stackrel{\simeq}{\longrightarrow} R \pi_{*} \widehat{\mathcal{D}}_{\mathfrak{X}^{\prime}, \mathbb{Q}}$. By passing to the cohomology sheaves (and to the inductive limit over all $m$ ), this proves (i). The part (ii) follows from the definition of the functor $\pi^{!}$, cf. 2.3.3.

We can now state the

Theorem 2.3.8. Let $\pi: \mathfrak{X}^{\prime} \rightarrow \mathfrak{X}$ be a morphism over $\mathfrak{X}_{0}$ between admissible formal blow-ups of the smooth formal scheme $\mathfrak{X}_{0}$. Let $k \geqslant \max \left\{k_{\mathfrak{X}}, k_{\mathfrak{X}^{\prime}}\right\}$.

(i) If $\mathcal{M}$ is a coherent $\mathcal{D}_{\mathfrak{X}^{\prime}, k}^{\dagger}$-module, then $R^{j} \pi_{*} \mathcal{M}=0$ for $j>0$. Moreover, $\pi_{*} \mathcal{D}_{\mathfrak{X}^{\prime}, k}^{\dagger}=$ $\mathcal{D}_{\mathfrak{X}, k}^{\dagger}$, so that $\pi_{*}$ induces an exact functor between coherent modules over $\mathcal{D}_{\mathfrak{X}^{\prime}, k}^{\dagger}$ and $\mathcal{D}_{\mathfrak{X}, k}^{\dagger}$ respectively.

(ii) The formation $\pi^{!}$is an exact functor from the category of coherent $\mathcal{D}_{\mathfrak{X}, k}^{\dagger}$-modules to the category of coherent $\mathcal{D}_{\mathfrak{X}^{\prime}, k}^{\dagger}$-modules, and $\pi^{!}$and $\pi_{*}$ are quasi-inverse equivalences between these categories.

The same statement holds for coherent modules over $\widehat{\mathcal{D}}_{\mathfrak{X}, \mathbb{Q}}^{(k, m)}$ and $\widehat{\mathcal{D}}_{\mathfrak{X}^{\prime}, \mathbb{Q}}^{(k, m)}$ respectively.

Proof. The first assertion of part (i) is true for $\mathcal{D}_{\mathfrak{X}^{\prime}, k}^{\dagger}$ by the previous lemma 2.3.7. Now there is a basis of the topology of $\mathfrak{X}$ consisting of affine opens $\mathfrak{V}$ such that $\operatorname{pr}(\mathfrak{V})$ is contained in some affine open of $\mathfrak{X}_{0}$. For this reason, if some statement is local on $\mathfrak{X}$, then we can assume that $\mathfrak{X}_{0}$ is affine. To prove (i) of the theorem for coherent $\mathcal{D}_{\mathfrak{X}^{\prime}, k}^{\dagger}$-modules, we can thus assume (and we do assume) that $\mathfrak{X}_{0}$ is affine. Then $\mathfrak{X}$ and $\mathfrak{X}^{\prime}$ are admissible blow-ups of a smooth affine formal scheme $\mathfrak{X}_{0}$ and we still call $\pi$ the map $\mathfrak{X}^{\prime} \rightarrow \mathfrak{X}$. We consider now the following assertion depending on $j$ :

For any coherent $\mathcal{D}_{\mathfrak{X}^{\prime}, k}^{\dagger}$-module $\mathcal{M}$, and for any $l \geqslant j$, one has $R^{l} \pi_{*} \mathcal{M}=0$.

We will prove this assertion for any $j \geqslant 1$ by decreasing induction on $j$. The statement for $j=1$ establishes then the first assertion of part (i) in general. Since $\mathfrak{X}^{\prime}$ has dimension $\leqslant M+1$, the assertion is true for $j=M+2$. Assume that the assertion is true for $j+1$ and take a coherent $\mathcal{D}_{\mathfrak{X}^{\prime}, k}^{\dagger}$-module $\mathcal{M}$. Since $\mathfrak{X}_{0}$ is affine, we can apply 2.2.29 to find a non negative integer $r$ and an exact sequence of coherent $\mathcal{D}_{\mathfrak{X}^{\prime}, k}^{\dagger}$-modules

$$
0 \rightarrow \mathcal{N} \rightarrow\left(\mathcal{D}_{\mathfrak{X}^{\prime}, k}^{\dagger}\right)^{r} \rightarrow \mathcal{M} \rightarrow 0
$$

Since $j \geqslant 1$ and since $R^{j} \pi_{*} \mathcal{D}_{\mathfrak{X}^{\prime}, k}^{\dagger}=0$ by lemma 2.3.7, the long exact sequence for $\pi_{*}$ gives us an isomorphism

$$
R^{j} \pi_{*} \mathcal{M} \simeq R^{j+1} \pi_{*} \mathcal{N} .
$$

But the right-hand side is zero by the induction hypothesis applied to $\mathcal{N}$. This establishes the assertion for $j$ and completes the induction step. This ends the proof of the first assertion of part (i). 
What remains to prove for part (i) is that $\pi_{*} \mathcal{M}$ is coherent over $\mathcal{D}_{\mathfrak{X}, k}^{\dagger}$ if $\mathcal{M}$ is coherent over $\mathcal{D}_{\mathfrak{X}^{\prime}, k}^{\dagger}$. To show this, we continue to assume that $\mathfrak{X}_{0}$ is affine. By 2.2.29, there is a finite presentation

$$
\left(\mathcal{D}_{\mathfrak{X}^{\prime}, k}^{\dagger}\right)^{s} \rightarrow\left(\mathcal{D}_{\mathfrak{X}^{\prime}, k}^{\dagger}\right)^{r} \rightarrow \mathcal{M} \rightarrow 0
$$

Applying $\pi_{*}$ and using that $\pi_{*}$ is exact, we obtain a finite presentation for $\pi_{*} \mathcal{M}$, which implies that the latter is coherent.

Let us prove part (ii) in the case of coherent $\mathcal{D}_{\mathfrak{X}, k}^{\dagger}$-modules, the case of coherent $\widehat{\mathcal{D}}_{\mathfrak{X}, \mathbb{Q}}^{(k, m)}$ modules can be treated analogously. By definition of the functor $\pi^{!}$, cf. 2.3.3, we have $\pi^{!} \mathcal{D}_{\mathfrak{X}, k}^{\dagger}=\mathcal{D}_{\mathfrak{X}^{\prime}, k}^{\dagger}$. To prove that $\pi^{!}$preserves coherence is local on $\mathfrak{X}$, so that we can (and will) again assume that $\mathfrak{X}_{0}$ is affine. Let $\mathcal{M}$ be a coherent $\mathcal{D}_{\mathfrak{X}, k}^{\dagger}$-module. We can apply proposition 2.2 .29 to $\mathfrak{X}$ and obtain a finite presentation

$$
\left(\mathcal{D}_{\mathfrak{X}, k}^{\dagger}\right)^{s} \rightarrow\left(\mathcal{D}_{\mathfrak{X}, k}^{\dagger}\right)^{r} \rightarrow \mathcal{M} \rightarrow 0
$$

Since the tensor product is right exact, we get a finite presentation of $\pi ! \mathcal{M}$

$$
\left(\mathcal{D}_{\mathfrak{X}^{\prime}, k}^{\dagger}\right)^{s} \rightarrow\left(\mathcal{D}_{\mathfrak{X}^{\prime}, k}^{\dagger}\right)^{r} \rightarrow \pi^{!} \mathcal{M} \rightarrow 0
$$

which implies that $\pi^{!} \mathcal{M}$ is a coherent $\mathcal{D}_{\mathfrak{X}^{\prime}, k}^{\dagger}$-module. In particular, the functor $\pi^{!}$preserves coherence. The map $\pi^{-1} \mathcal{M} \rightarrow \pi^{!} \mathcal{M}$ sending $x$ to $1 \otimes x$ induces a morphism

$$
\operatorname{can}_{\mathcal{M}}: \mathcal{M} \rightarrow \pi_{*} \pi^{!} \mathcal{M},
$$

which is natural in $\mathcal{M}$. Whether can $_{\mathcal{M}}$ is an isomorphism can be decided locally on $\mathfrak{X}$, and we can again assume that $\mathfrak{X}_{0}$ is affine. $\mathfrak{X}$ and $\mathfrak{X}^{\prime}$ are admissible blow-ups of $\mathfrak{X}_{0}$, and by 2.2 .29 there a finite presentation

$$
\left(\mathcal{D}_{\mathfrak{X}, k}^{\dagger}\right)^{s} \rightarrow\left(\mathcal{D}_{\mathfrak{X}, k}^{\dagger}\right)^{r} \rightarrow \mathcal{M} \rightarrow 0
$$

and so $\pi ! \mathcal{M}$ admits a finite presentation

$$
\left(\mathcal{D}_{\mathfrak{X}^{\prime}, k}^{\dagger}\right)^{s} \rightarrow\left(\mathcal{D}_{\mathfrak{X}^{\prime}, k}^{\dagger}\right)^{r} \rightarrow \pi ! \mathcal{M} \rightarrow 0
$$

We apply $\pi_{*}$ to this latter sequence and use that $\pi_{*}$ is exact (by (i)), together with 2.3.7 to obtain the finite presentation

$$
\left(\mathcal{D}_{\mathfrak{X}, k}^{\dagger}\right)^{s} \rightarrow\left(\mathcal{D}_{\mathfrak{X}, k}^{\dagger}\right)^{r} \rightarrow \pi_{*} \pi^{!} \mathcal{M} \rightarrow 0
$$

The natural transformation can induces a morphism from 2.3.9 to 2.3.10, and because $\operatorname{can}_{\mathcal{D}_{\mathfrak{X}, k}^{\dagger}}$ is an isomorphism, so is $\operatorname{can}_{\mathcal{M}}$.

In the reverse direction, let $\mathcal{M}^{\prime}$ be a coherent $\mathcal{D}_{\mathfrak{X}^{\prime}, k}^{\dagger}$-module. There is a map $c a n_{\mathcal{N}^{\prime}}^{\prime}$ : $\pi^{!} \pi_{*} \mathcal{M}^{\prime} \rightarrow \mathcal{M}^{\prime}$, sending $P \otimes x$ to $P x$, which is natural in $\mathcal{M}^{\prime}$. Whether this map is bijective can be decided locally on $\mathfrak{X}$ and we may assume that $\mathfrak{X}_{0}$ is affine, and $\mathfrak{X}^{\prime}$ and $\mathfrak{X}$ are admissible blow-ups of $\mathfrak{X}_{0}$. Since $\pi_{*}$ is exact, and using 2.2.29] over $\mathfrak{X}^{\prime}$, we are reduced 
to the case where $\mathcal{M}^{\prime}=\mathcal{D}_{\mathfrak{X}^{\prime}, k}^{\dagger}$. In this case $\pi^{!} \pi_{*} \mathcal{D}_{\mathfrak{X}^{\prime}, k}^{\dagger} \simeq \mathcal{D}_{\mathfrak{X}^{\prime}, k}^{\dagger}$ by (i). From all of this, we can conclude that $\pi^{!}$and $\pi_{*}$ are quasi-inverse functors. As $\pi_{*}$ is exact on coherent $\mathcal{D}_{\mathfrak{X}^{\prime}, k}^{\dagger}$-modules, $\pi^{!}$is exact on coherent $\mathcal{D}_{\mathfrak{X}, k}^{\dagger}$-modules.

Corollary 2.3.11. In the situation of the preceding theorem, one has

$$
\Gamma\left(\mathfrak{X}, \mathcal{D}_{\mathfrak{X}, k}^{\dagger}\right)=\Gamma\left(\mathfrak{X}_{0}, \mathcal{D}_{\mathfrak{X}_{0}, k}^{\dagger}\right)=\Gamma\left(\mathfrak{X}^{\prime}, \mathcal{D}_{\mathfrak{X}^{\prime}, k}^{\dagger}\right) \text {. }
$$

As an application of the invariance theorem we can extend the local theorems $\mathrm{A}$ and $\mathrm{B}$ 2.2 .6 and 2.2.15 to global statements, provided that the base $\mathfrak{X}_{0}$ is affine 3 .

Theorem 2.3.12. (Global theorem $\mathrm{A}$ and $\mathrm{B}$ over an affine base) Let $\mathfrak{X}_{0}$ be affine.

(i) For any coherent $\widehat{\mathcal{D}}_{\mathfrak{X}, \mathbb{Q}}^{(k, m)}$-module $\mathcal{M}$ and for all $q>0$ one has $H^{q}(\mathfrak{X}, \mathcal{M})=0$.

(ii) The functor $\Gamma(\mathfrak{X},-)$ is an equivalence between the category of coherent $\widehat{\mathcal{D}}_{\mathfrak{X}, \mathbb{Q}}^{(k, m)}$ modules and the category of coherent $\Gamma\left(\mathfrak{X}, \widehat{\mathcal{D}}_{\mathfrak{X}, \mathbb{Q}}^{(k, m)}\right)$-modules.

The same statement holds for coherent modules over $\mathcal{D}_{\mathfrak{X}, k}^{\dagger}$ and $\Gamma\left(\mathfrak{X}, \mathcal{D}_{\mathfrak{X}, k}^{\dagger}\right)$.

Proof. Denote by $\pi: \mathfrak{X} \rightarrow \mathfrak{X}_{0}$ the blow-up morphism. The functor $\Gamma(\mathfrak{X},$.$) equals the$ composite of the two functors $\pi_{*}$ and $\Gamma\left(\mathfrak{X}_{0},-\right)$. Hence the theorem follows from 2.3.8 and its corollary 2.3.11 and 2.2.15,

\section{Coadmissible $\mathcal{D}$-modules on $\mathfrak{X}$ and the Zariski-Riemann space}

We continue to denote throughout this section by $\mathfrak{X}_{0}$ a smooth formal $\mathfrak{S}$-scheme, and we consider an admissible formal blow-up

$$
\text { pr }: \mathfrak{X} \rightarrow \mathfrak{X}_{0} \text {. }
$$

The purpose of the first subsection is to study projective systems $\left(\mathcal{M}_{k}\right)_{k \geqslant k_{\mathfrak{X}}}$ of coherent modules $\mathcal{M}_{k}$ over $\mathcal{D}_{\mathfrak{X}, k}^{\dagger}$, and to pass to their associated projective limits. In the second subsection we will then let $\mathfrak{X}$ vary in the system of all admissible formal blow-ups of $\mathfrak{X}_{0}$.

3.1. Coadmissible $\mathcal{D}$-modules on $\mathfrak{X}$. We make the general convention that $k$ always denotes an integer which is at least as large as $k_{\mathfrak{X}}$.

3.1.1. Fréchet-Stein algebras. Let $B$ be a noetherian $K$-Banach algebra. We recall that any finitely generated $B$-module has a canonical structure as $B$-Banach module and any $B$-linear map between two such modules is continuous. The topology can be defined as the quotient topology with respect to any chosen finite presentation of the module [23, Prop. 2.1].

We recall from [23, sec. 3] that a $K$-Fréchet algebra $A$ is called Fréchet-Stein if there is a projective system $\left(A_{k}, A_{k+1} \rightarrow A_{k}\right)_{k \in \mathbb{N}}$ of (left) noetherian $K$-Banach algebras $A_{k}$ with

\footnotetext{
${ }^{3}$ Note that this is not covered by 2.2.6 and 2.2.15 since an admissible blow-up $\mathfrak{X}$ of an affine $\mathfrak{X}_{0}$ is in general not affine.
} 
(right) flat transition maps $A_{k+1} \rightarrow A_{k}$, and an isomorphism of topological $K$-algebras $A \simeq \lim _{k} A_{k}$. For the following definition we fix such an isomorphism. We denote by $\mathcal{C}_{A}$ the full abelian subcategory of the category of all (left) $A$-modules consisting of the coadmissible $A$-modules, as introduced in [23]. For an $A$-module $M$ to be coadmissible means that there is a projective system $\left(M_{k}, M_{k+1} \rightarrow M_{k}\right)_{k \in \mathbb{N}}$, where each $M_{k}$ is a finitely generated $A_{k}$-module, such that

(i) the transition map $M_{k+1} \rightarrow M_{k}$ is a homomorphism of $A_{k+1}$-modules, and the induced map $A_{k} \otimes_{A_{k+1}} M_{k+1} \rightarrow M_{k}$ is an isomorphism of $A_{k}$-modules, and

(ii) $M$ is isomorphic to $\lim _{k} M_{k}$ as an $A$-module.

(The projective limit is considered as an $A$-module via the fixed isomorphism $A \simeq$ $\lim _{k} A_{k}$.) We sometimes call $\left(M_{k}\right)$ an $\left(A_{k}\right)$-sequence for $M$. For $M=\lim _{k} M_{k} \in \mathcal{C}_{A}$ we have that the image of $M \rightarrow M_{k}$ is dense with respect to the canonical topology for any $k$, and $\lim _{k}^{(1)} M_{k}=0$, cf. [23, first theorem in sec. 3].

3.1.2. The sheaf $\mathcal{D}_{\mathfrak{X}, \infty}$. We denote by

$$
\mathcal{D}_{\mathfrak{X}, \infty}=\lim _{k} \mathcal{D}_{\mathfrak{X}, k}^{\dagger}
$$

the projective limit of the system of sheaves $\mathcal{D}_{\mathfrak{X}, k}^{\dagger}$. Then $\mathcal{D}_{\mathfrak{X}, \infty}$ is again a sheaf of rings and for every open subset $\mathfrak{V} \subset \mathfrak{X}$ we have $\mathcal{D}_{\mathfrak{X}, \infty}(\mathfrak{V})=\varliminf_{\lim _{k}} \mathcal{D}_{\mathfrak{X}, k}^{\dagger}(\mathfrak{V})$.

\section{Proposition 3.1.4.}

(i) The canonical morphism of sheaves $\widehat{\mathcal{D}}_{\mathfrak{X}, \mathbb{Q}}^{(k, 0)} \rightarrow \mathcal{D}_{\mathfrak{X}, k}^{\dagger}$ induces an isomorphism

$$
\lim _{k} \widehat{\mathcal{D}}_{\mathfrak{X}, \mathbb{Q}}^{(k, 0)} \stackrel{\simeq}{\longleftarrow} \lim _{k} \mathcal{D}_{\mathfrak{X}, k}^{\dagger}=\mathcal{D}_{\mathfrak{X}, \infty} \text {. }
$$

(ii) For every affine open subset $\mathfrak{V} \subset \mathfrak{X}$ the isomorphism

$$
\mathcal{D}_{\mathfrak{X}, \infty}(\mathfrak{V})=\lim _{k} \widehat{\mathcal{D}}_{\mathfrak{X}, \mathbb{Q}}^{(k, 0)}(\mathfrak{V})
$$

induced from (i) gives $\mathcal{D}_{\mathfrak{X}, \infty}(\mathfrak{V})$ the structure of a Fréchet-Stein algebra.

(iii) Let $\mathfrak{U} \subset \mathfrak{X}_{0}$ be an open affine subset which can be equipped with a system of étale coordinates $x_{1}, \ldots, x_{M}$ and $\partial_{1}, \ldots, \partial_{M}$ the corresponding derivations. Then, for any affine open $\mathfrak{V} \subset \operatorname{pr}^{-1}(\mathfrak{U})$ we have

$$
\mathcal{D}_{\mathfrak{X}, \infty}(\mathfrak{V})=\left\{\sum_{\underline{\nu}} a_{\underline{\nu}} \underline{\partial}^{\underline{\nu}} \mid a_{\underline{\nu}} \in \mathcal{O}_{\mathfrak{X}, \mathbb{Q}}(\mathfrak{V}), \forall R>0: \lim _{|\underline{\nu}| \rightarrow \infty}\left\|a_{\underline{\underline{\nu}}}\right\| R^{|\underline{\nu}|}=0\right\},
$$

where $\|\cdot\|$ is any submultiplicative Banach space norm on $\mathcal{O}_{\mathfrak{X}, \mathbb{Q}}(\mathfrak{V})$.

\footnotetext{
${ }^{4}$ However, the category $\mathcal{C}_{A}$ is independent of the isomorphism.
} 
(iv) Let $\mathfrak{X}^{\prime} \rightarrow \mathfrak{X}_{0}$ be another admissible formal blow-up, and let $\pi: \mathfrak{X}^{\prime} \rightarrow \mathfrak{X}$ be a morphism over $\mathfrak{X}_{0}$. Then the canonical isomorphisms $\pi_{*} \mathcal{D}_{\mathfrak{X}^{\prime}, k}^{\dagger}=\mathcal{D}_{\mathfrak{X}, k}^{\dagger}$, for $k \geqslant \max \left\{k_{\mathfrak{X}^{\prime}}, k_{\mathfrak{X}}\right\}$, cf. 2.3.8, give rise to a canonical isomorphism

$$
\pi_{*} \mathcal{D}_{\mathfrak{X}^{\prime}, \infty}=\mathcal{D}_{\mathfrak{X}, \infty} \text {. }
$$

Proof. Take an affine open $\mathfrak{V} \subset \mathfrak{X}$. We deduce from the proposition 2.2 .22 that the projective systems of $K$-algebras $\mathcal{D}_{\mathfrak{X}, k}^{\dagger}(\mathfrak{V})$ and $\widehat{\mathcal{D}}_{\mathfrak{X}, \mathbb{Q}}^{(k, 0)}(\mathfrak{V})$ are equivalent. This proves (i). For (ii), note that the transition map $\widehat{\mathcal{D}}_{\mathfrak{X}, \mathbb{Q}}^{(k+1,0)}(\mathfrak{V}) \rightarrow \widehat{\mathcal{D}}_{\mathfrak{X}, \mathbb{Q}}^{(k, 0)}(\mathfrak{V})$ is a flat homomorphism between noetherian Banach algebras, according to propositions 2.2.16 and 2.2.2. To prove (iii), we assume additionally $\mathfrak{V} \subset \mathrm{pr}^{-1} \mathfrak{U}$. By part (i), is then enough to show

$$
\lim _{k} \widehat{\mathcal{D}}_{\mathfrak{X}, \mathbb{Q}}^{(k, 0)}(\mathfrak{V})=\left\{\sum_{\underline{\nu}} a_{\underline{\nu}} \underline{\underline{\partial}} \underline{\underline{\nu}} \mid a_{\underline{\nu}} \in \mathcal{O}_{\mathfrak{X}, \mathbb{Q}}(\mathfrak{V}), \forall R>0: \lim _{|\underline{\nu}| \rightarrow \infty}\left\|a_{\underline{\nu}}\right\| R^{|\underline{\nu}|}=0\right\} .
$$

Denote by $E$ the right-hand side of the preceding equality. Recall that

$$
\widehat{\mathcal{D}}_{\mathfrak{X}, \mathbb{Q}}^{(k, 0)}(\mathfrak{V})=\left\{\sum_{\underline{\nu}} \varpi^{k \mid \underline{\nu}} b_{\underline{\nu}} \underline{\partial}^{\underline{\nu}} \mid b_{\underline{\nu}} \in \mathcal{O}_{\mathfrak{X}, \mathbb{Q}}(\mathfrak{V}),\left\|b_{\underline{\nu}}\right\| \rightarrow 0\right\} .
$$

Let $P=\sum_{\underline{\nu}} a_{\underline{\nu}} \underline{\partial}^{\underline{\nu}} \in E$. Since all Banach algebra norms over $\mathcal{O}_{\mathfrak{X}, \mathbb{Q}}(\mathfrak{V})$ are equivalent, we can use the $p$-adic norm $|\cdot|_{p}$ of $\mathcal{O}_{\mathfrak{X}, \mathbb{Q}}(\mathfrak{V})$ relatively to the lattice $\mathcal{O}_{\mathfrak{X}}(\mathfrak{V})$. Fix $k \in \mathbb{N}$ and define

$$
b_{\underline{\nu}}=\varpi^{-k|\underline{\nu}|} a_{\underline{\nu}},
$$

then, using the ramification index $e$ of the extension $L / \mathbb{Q}_{p}$, we get that

$$
\left|b_{\underline{\nu}}\right|_{p}=\left|a_{\underline{\nu}}\right|_{p} p^{\frac{k \mid \underline{\underline{\nu}}}{e}} \rightarrow 0 \text { if }|\underline{\nu}| \rightarrow+\infty .
$$

Thus $P=\sum_{\underline{\nu}} \varpi^{k \mid \underline{\nu}} b_{\underline{\nu}} \underline{\partial}^{\underline{\nu}} \in \widehat{\mathcal{D}}_{\mathfrak{X}, \mathbb{Q}}^{(k, 0)}(\mathfrak{V})$. Conversely, let $P=\sum_{\underline{\nu}} a_{\underline{\nu}} \underline{\underline{\partial}} \underline{\underline{\nu}} \in \lim _{k} \widehat{\mathcal{D}}_{\mathfrak{X}, \mathbb{Q}}^{(k, 0)}(\mathfrak{V})$ and $R>0$. Choose $k>0$ such that

$$
p^{\frac{k}{e}}>R \text { and define } b_{\underline{\nu}}=\varpi^{-k|\underline{\underline{ }}|} a_{\underline{\nu}} .
$$

Since $P=\sum_{\underline{\nu}} \varpi^{k \mid \underline{\nu}} b_{\underline{\nu}} \underline{\partial}^{\underline{\nu}} \in \widehat{\mathcal{D}}_{\mathfrak{X}, \mathbb{Q}}^{(k, 0)}(\mathfrak{V}),\left|b_{\underline{\nu}}\right|_{p} \rightarrow 0$, thus

$$
\left|a_{\underline{\nu}}\right|_{p} p^{\frac{k \mid \underline{\underline{\nu}}}{e}} \rightarrow 0, \text { and }\left|a_{\underline{\nu}}\right|_{p} R^{|\underline{\mid \underline{ }}|} \rightarrow 0
$$

proving that $P \in E$, as required for (iii).

Let us prove (iv). Let $\mathfrak{V} \subset \mathfrak{X}$, then from part (i) of proposition 2.3.8, we know that $\mathcal{D}_{\mathfrak{X}, k}^{\dagger}(\mathfrak{V})=\mathcal{D}_{\mathfrak{X}^{\prime}, k}^{\dagger}\left(\pi^{-1}(\mathfrak{V})\right)$. We deduce from this the equations

$$
\pi_{*} \mathcal{D}_{\mathfrak{X}^{\prime}, \infty}(\mathfrak{V})=\mathcal{D}_{\mathfrak{X}^{\prime}, \infty}\left(\pi^{-1}(\mathfrak{V})\right)=\underbrace{\lim }_{k} \mathcal{D}_{\mathfrak{X}^{\prime}, k}^{\dagger}\left(\pi^{-1}(\mathfrak{V})\right)={\underset{l}{\longleftarrow}}_{k} \mathcal{D}_{\mathfrak{X}, k}^{\dagger}(\mathfrak{V})=\mathcal{D}_{\mathfrak{X}, \infty}(\mathfrak{V}) \text {. }
$$


For every affine open subset $\mathfrak{V} \subset \mathfrak{X}$ we have, according to the preceding proposition, the abelian category of coadmissible $\mathcal{D}_{\mathfrak{X}, \infty}(\mathfrak{V})$-modules $\mathcal{C}_{\mathcal{D}_{\mathfrak{X}, \infty}(\mathfrak{V})}$. We give an alternative description of these modules using the projective system of algebras $\mathcal{D}_{\mathfrak{X}, k}^{\dagger}(\mathfrak{V})$.

Proposition 3.1.7. Let $\mathfrak{V} \subset \mathfrak{X}$ be an affine open. A $\mathcal{D}_{\mathfrak{X}, \infty}(\mathfrak{V})$-module $M$ is coadmissible if and only if there is a projective system $\left(M_{k}, M_{k+1} \rightarrow M_{k}\right)$, where each $M_{k}$ is a finitely presented $\mathcal{D}_{\mathfrak{X}, k}^{\dagger}(\mathfrak{V})$-module, such that

(i) the transition map $M_{k+1} \rightarrow M_{k}$ is $\mathcal{D}_{\mathfrak{X}, k+1}^{\dagger}(\mathfrak{V})$-linear and induces an isomorphism

$$
\mathcal{D}_{\mathfrak{X}, k}^{\dagger}(\mathfrak{V}) \otimes_{\mathcal{D}_{\mathfrak{X}, k+1}^{\dagger}(\mathfrak{V})} M_{k+1} \simeq M_{k}
$$

of $\mathcal{D}_{\mathfrak{X}, k}^{\dagger}(\mathfrak{V})$-modules, and

(ii) $M$ is isomorphic to $\lim _{k} M_{k}$ as an $\mathcal{D}_{\mathfrak{X}, \infty}(\mathfrak{V})$-module.

Proof. This follows from the discussion given in the proof of [10, Prop. 1.2.9] and we explain the main points. We write $A_{k}=\widehat{\mathcal{D}}_{\mathfrak{X}, \mathbb{Q}}^{(k, 0)}(\mathfrak{V})$ and $B_{k}=\mathcal{D}_{\mathfrak{X}, k}^{\dagger}(\mathfrak{V})$. We have strictly increasing functions $\phi$ and $\psi$ mapping $\mathbb{N}$ to itself such that the map $A \rightarrow A_{k}$ (resp. $A \rightarrow B_{k}$ ) factors through the map $A \rightarrow B_{\phi(k)}$ (resp. $\left.A \rightarrow A_{\psi(k)}\right)$. Indeed, we may take $\phi(k)=k+e^{\prime}$ and $\psi(k)=k$ with $e^{\prime} \geqslant \frac{e}{p-1}$ a fixed number, according to the proposition 2.2.22. In particular, the systems of $K$-algebras $A_{k}$ and $B_{k}$ are equivalent. Now suppose $N$ is coadmissible with system of Banach modules $N_{k}$. For $k \geqslant 1$ define the finitely presented $B_{k}$-module

$$
M_{k}=B_{k} \otimes_{A_{\psi(k)}} N_{\psi(k)} .
$$

Since $\psi(k+1) \geqslant \psi(k)$, the map $N_{\psi(k+1)} \rightarrow N_{\psi(k)}$ induces a map $M_{k+1} \rightarrow M_{k}$ and then a map $B_{k} \otimes_{B_{k+1}} M_{k+1} \rightarrow M_{k}$. This map is bijective as follows from the diagram presented in the proof of [10, Prop. 1.2.9]. Moreover, the projection map $M \rightarrow N_{\psi(k)}$ induces a A-linear map

$$
M \rightarrow B_{k} \otimes_{A} M \rightarrow B_{k} \otimes_{A_{\psi(k)}} N_{\psi(k)}=M_{k}
$$

compatible with $M_{k+1} \rightarrow M_{k}$. This gives an $A$-linear map $M \rightarrow \lim _{k} M_{k}$ and it remains to see that it is bijective. We have the natural map $N_{\psi(k)} \rightarrow M_{k}$. On the other hand, $\psi(\phi(k)) \geqslant k$ such that there is a map

$$
M_{\phi(k)}=B_{\phi(k)} \otimes_{A_{\psi(\phi(k))}} N_{\psi(\phi(k))} \rightarrow A_{k} \otimes_{A_{\psi(\phi(k))}} N_{\psi(\phi(k))} \rightarrow N_{k}
$$

using the map $B_{\phi(k)} \rightarrow A_{k}$. Hence the systems $\left(M_{k}\right)$ and $\left(N_{k}\right)$ are equivalent and

$$
M \simeq \lim _{k} N_{k} \simeq \lim _{k} M_{k}
$$

This shows that the system $\left(M_{k}\right)$ is as required for $M$. Conversely, starting with a module $M$ and such a system $\left(M_{k}\right)$ the coadmissibility of $M$ follows with the same argument.

Definition 3.1.8. A $\mathcal{D}_{\mathfrak{X}, \infty}$-module $\mathcal{M}$ is called coadmissible if there a projective system $\left(\mathcal{M}_{k}, \mathcal{M}_{k+1} \rightarrow \mathcal{M}_{k}\right)_{k \geqslant k_{\mathfrak{X}}}$, where $\mathcal{M}_{k}$ is a coherent $\widehat{\mathcal{D}}_{\mathfrak{X}, \mathbb{Q}}^{(k, 0)}$-module, such that 
(i) the transition map $\mathcal{M}_{k+1} \rightarrow \mathcal{M}_{k}$ is $\widehat{\mathcal{D}}_{\mathfrak{X}, \mathbb{Q}}^{(k+1,0)}$-linear and the induced map

$$
\widehat{\mathcal{D}}_{\mathfrak{X}, \mathbb{Q}}^{(k, 0)} \otimes_{\hat{\mathcal{D}}_{\mathfrak{X}, \mathbb{Q}}^{(k+1,0)}} \mathcal{M}_{k+1} \longrightarrow \mathcal{M}_{k}
$$

is an isomorphism as $\widehat{\mathcal{D}}_{\mathfrak{X}, \mathbb{Q}}^{(k, 0)}$-modules, and

(ii) $\mathcal{M}$ is isomorphic to $\lim _{k} \mathcal{M}_{k}$ as $\mathcal{D}_{\mathfrak{X}, \infty}$-module.

We denote by

$$
\mathcal{C}_{\mathfrak{X}} \subseteq \operatorname{Mod}\left(\mathcal{D}_{\mathfrak{X}, \infty}\right)
$$

the full subcategory of coadmissible $\mathcal{D}_{\mathfrak{X}, \infty}$-modules in the category of all $\mathcal{D}_{\mathfrak{X}, \infty}$-modules.

Proposition 3.1.10. A $\mathcal{D}_{\mathfrak{X}, \infty}$-module $\mathcal{M}$ is coadmissible if and only if a projective system $\left(\mathcal{M}_{k}, \mathcal{M}_{k+1} \rightarrow \mathcal{M}_{k}\right)_{k \geqslant k_{\mathfrak{X}}}$, where $\mathcal{M}_{k}$ is a coherent $\mathcal{D}_{\mathfrak{X}, k}^{\dagger}$-module, such that

(i) the transition map $\mathcal{M}_{k+1} \rightarrow \mathcal{M}_{k}$ is $\mathcal{D}_{\mathfrak{X}, k+1}^{\dagger}$-linear and the induced morphism of sheaves

$$
\mathcal{D}_{\mathfrak{X}, k}^{\dagger} \otimes_{\mathcal{D}_{\mathfrak{X}, k+1}^{\dagger}} \mathcal{M}_{k+1} \longrightarrow \mathcal{M}_{k}
$$

is an isomorphism of $\mathcal{D}_{\mathfrak{X}, k}^{\dagger}$-modules, and

(ii) $\mathcal{M}$ is isomorphic to $\lim _{k} \mathcal{M}_{k}$ as $\mathcal{D}_{\mathfrak{X}, \infty}$-module.

Proof. This follows literally as for the modules of local sections, cf. proposition 3.1.7 taking into account that proposition 2.2 .22 holds on the level of sheaves.

Theorem 3.1.12. (Theorem A for coadmissible modules on $\mathfrak{X}$ ) Let $\mathfrak{V} \subset \mathfrak{X}$ be an affine open subset. Then the global sections functor $\Gamma(\mathfrak{V},-)$ induces an equivalence of categories

$$
\Gamma(\mathfrak{V},-): \mathcal{C}_{\mathfrak{V}} \stackrel{\simeq}{\longrightarrow} \mathcal{C}_{\mathcal{D}_{\mathfrak{X}, \infty}(\mathfrak{V})} .
$$

Proof. This is an application of proposition 2.2.15, Abbreviate $D_{k}^{\dagger}=\Gamma\left(\mathfrak{V}, \mathcal{D}_{\mathfrak{V}, k}^{\dagger}\right)$. Let $\mathcal{M} \in \mathcal{C}_{\mathfrak{V}}$ with coherent $\mathcal{D}_{\mathfrak{X}, k}^{\dagger}$-modules $\mathcal{M}_{k}$. Then $M_{k}=\Gamma\left(\mathfrak{V}, \mathcal{M}_{k}\right)$ is a coherent $D_{k}^{\dagger}$-module and $M=\Gamma(\mathfrak{V}, \mathcal{M})=\lim _{k} M_{k}$. Taking global sections in the isomorphism

$$
\mathcal{D}_{\mathfrak{X}, k}^{\dagger} \otimes_{\mathcal{D}_{\mathfrak{X}, k+1}^{\dagger}} \mathcal{M}_{k+1} \stackrel{\simeq}{\longrightarrow} \mathcal{M}_{k}
$$

shows that the canonical map

$$
\mathcal{D}_{k}^{\dagger} \otimes_{\mathcal{D}_{k+1}^{\dagger}} M_{k+1} \stackrel{\simeq}{\longrightarrow} M_{k}
$$

is an isomorphism too. Indeed, this is clear in the case $\mathcal{M}_{k+1}=\mathcal{D}_{\mathfrak{V}, k+1}^{\dagger}$ and the general case follows from taking a finite presentation of $\mathcal{M}_{k+1}$ as $\mathcal{D}_{\mathfrak{V}, k+1}^{\dagger}$-module. We conclude with proposition 3.1 .7 that $M \in \mathcal{C}_{\mathcal{D}_{\mathfrak{X}, \infty}(\mathfrak{V})}$. Conversely, given $M \in \mathcal{C}_{\mathcal{D}_{\mathfrak{x}, \infty}(\mathfrak{V})}$ with coherent $D_{k}^{\dagger}$-modules $M_{k}$ the $\mathcal{D}_{\mathfrak{V}, k}^{\dagger}$-module $\mathcal{M}_{k}=\mathcal{D}_{\mathfrak{V}, k}^{\dagger} \otimes_{D_{k}^{\dagger}} M_{k}$ is coherent and these modules satisfy 3.1.13. Indeed, that the canonical map 3.1.13 is an isomorphism can be checked 
on global sections and follows then from the compatibility with the tensor product. This shows that $\mathcal{M}=\lim _{k} \mathcal{M}_{k}$ lies in $\mathcal{C}_{\mathfrak{V}}$. This provides a quasi-inverse to $\Gamma(\mathfrak{V},-)$.

Lemma 3.1.14. Let $\mathcal{M} \in \mathcal{C}_{\mathfrak{X}}$ with sequence $\left(\mathcal{M}_{k}\right)$ of coherent $\widehat{\mathcal{D}}_{\mathfrak{X}, \mathbb{Q}}^{(k, 0)}$-modules. Let $\mathfrak{V} \subset$ $\mathfrak{X}$ be an open affine subset. Then the projective system $\left(\mathcal{M}_{k}(\mathfrak{V})\right)_{k \geqslant k_{\mathfrak{X}}}$ has the following properties:

(i) For $k^{\prime} \geqslant k$ the transition map $\mathcal{M}_{k^{\prime}}(\mathfrak{V}) \rightarrow \mathcal{M}_{k}(\mathfrak{V})$ is uniformly continuous.

(ii) For all $k \geqslant k_{\mathfrak{X}}$ there exists $k^{\prime} \geqslant k$ such that for all $k^{\prime \prime} \geqslant k^{\prime}$ the image of $\mathcal{M}_{k^{\prime \prime}}(\mathfrak{V}) \rightarrow$ $\mathcal{M}_{k}(\mathfrak{U})$ is dense in $\operatorname{im}\left(\mathcal{M}_{k^{\prime}}(\mathfrak{V}) \rightarrow \mathcal{M}_{k}(\mathfrak{V})\right)$.

(iii) $\lim _{k}^{(1)} \mathcal{M}_{k}(\mathfrak{V})=0$.

Proof. Since a continuous map between Banach spaces is uniformly continuous, (i) is clear. Abbreviate $D_{k}=\Gamma\left(\mathfrak{V}, \widehat{\mathcal{D}}_{\mathfrak{X}, \mathbb{Q}}^{(k, 0)}\right)$ and $M_{k}=\Gamma\left(\mathfrak{V}, \mathcal{M}_{k}\right)$. By the above theorem $M \in \mathcal{C}_{\mathcal{D}_{\mathfrak{X}, \infty}(\mathfrak{V})}$ and, by the general properties of Fréchet-Stein algebras which we have recalled above, it remains to see that the modules $M_{k}=\mathcal{M}_{k}(\mathfrak{V})$ form a $\left(D_{k}\right)$-sequence for $M$. This is an application of proposition 2.2.6. First, $M_{k}$ is a coherent $D_{k}$-module. Applying $\Gamma(\mathfrak{V},-)$ to the isomorphism

shows that the canonical map

$$
\widehat{\mathcal{D}}_{\mathfrak{X}, \mathbb{Q}}^{(k, 0)} \otimes_{\hat{\mathcal{D}}_{\mathfrak{X}, \mathbb{Q}}^{(k+1,0)}} \mathcal{M}_{k+1} \stackrel{\simeq}{\longrightarrow} \mathcal{M}_{k}
$$

$$
\mathcal{D}_{k} \otimes_{\mathcal{D}_{k+1}} M_{k+1} \stackrel{\simeq}{\longrightarrow} M_{k}
$$

is an isomorphism. Indeed, this is clear in the case where the restriction of $\mathcal{M}_{k+1}$ to $\mathfrak{V}$ equals $\widehat{\mathcal{D}}_{\mathfrak{V}, \mathbb{Q}}^{(k, 0)}$ and the general case follows from taking a finite presentation of it as $\widehat{\mathcal{D}}_{\mathfrak{V}, \mathbb{Q}}^{(k, 0)}$-module.

Theorem 3.1.15. (Theorem $B$ for coadmissible modules on $\mathfrak{X}$ ) For every coadmissible $\mathcal{D}_{\mathfrak{X}, \infty}$-module $\mathcal{M}$ and for all $q>0$ one has $H^{q}(\mathfrak{X}, \mathcal{M})=0$. Moreover, if $\mathcal{M}=\lim _{k} \mathcal{M}_{k}$ with coherent $\widehat{\mathcal{D}}_{\mathfrak{X}, k}^{(k, 0)}$-modules $\mathcal{M}_{k}$, then $R \lim _{k} \mathcal{M}_{k}=\mathcal{M}$.

Proof. Our aim is to apply [25, 20.32.4]. To this end, we let $\mathcal{B}$ be the set of open affine subsets $\mathfrak{V} \subset \mathfrak{X}$ which are contained in a subset of the form $\operatorname{pr}^{-1}(\mathfrak{U})$ for some open affine $\mathfrak{U} \subset \mathfrak{X}_{0}$. We are going to show that the three hypotheses of loc.cit. are fulfilled, namely

(i) Every open subset of $\mathfrak{X}$ has a covering whose members are elements of $\mathcal{B}$.

(ii) For every $\mathfrak{V} \in \mathcal{B}$, all $k \geqslant 0$, and all $q>0$ one has $H^{q}\left(\mathfrak{V}, \mathcal{M}_{k}\right)=0$.

(iii) For every $\mathfrak{V} \in \mathcal{B}$ one has $\lim _{k}^{(1)} \mathcal{M}_{k}(\mathfrak{V})=0$.

Proof of these conditions. (i) This is true because $\mathcal{B}$ is a basis of the topology of $\mathfrak{X}$.

(ii) This is true by 2.2.6 (ii).

(iii) This is true by 3.1 .14 
This shows that the conclusions of [25, 20.32.4] hold, namely that $R \lim _{k} \mathcal{M}_{k}=\mathcal{M}$, and that $H^{q}(\mathfrak{X}, \mathcal{M})=0$ for all $q>0$.

Corollary 3.1.16. The category $\mathcal{C}_{\mathfrak{X}}$ is abelian.

Proof. Let $f_{k}: \mathcal{M} \rightarrow \mathcal{N}$ be a morphism in $\mathcal{C}_{\mathfrak{X}}$ and denote by $\mathcal{K}=\operatorname{ker}(f)$ and $\mathcal{J}=\operatorname{im}(f)$ its kernel and image in $\operatorname{Mod}\left(\mathcal{D}_{\mathfrak{X}, \infty}\right)$. By left-exactness of the projective limit, we have $\mathcal{K}=\lim _{k} \mathcal{K}_{k}$ where $\mathcal{K}_{k}=\operatorname{ker}\left(f_{k}\right)$ and a linear map

$$
\mathcal{D}_{\mathfrak{X}, k}^{\dagger} \otimes_{\mathcal{D}_{\mathfrak{X}, k+1}^{\dagger}} \mathcal{K}_{k+1} \longrightarrow \mathcal{K}_{k}
$$

Let $\mathfrak{V} \subseteq \mathfrak{X}$ be an open affine. By the preceding theorem, we know that $\mathcal{K}(\mathfrak{V}) \in \mathcal{C}_{\mathcal{D}_{\mathfrak{X}, \infty}(\mathfrak{V})}$. Since the morphism 3.1.17, restricted to $\mathfrak{V}$, is a morphism of coherent $\mathcal{D}_{\mathfrak{V}, k}^{\dagger}$-modules, we may test its bijectivity on global sections according to proposition 2.2.6. Applying $\Gamma(\mathfrak{V},-)$ to 3.1 .17 yields

$$
\mathcal{D}_{k}^{\dagger} \otimes_{\mathcal{D}_{k+1}^{\dagger}} \mathcal{K}_{k+1}(\mathfrak{V}) \longrightarrow \mathcal{K}_{k}(\mathfrak{V})
$$

where $\mathcal{D}_{k}^{\dagger}=\mathcal{D}_{\mathfrak{X}, k}^{\dagger}(\mathfrak{V})$. Indeed, this is clear in the case where the restriction of $\mathcal{K}_{k+1}$ to $\mathfrak{V}$ equals $\mathcal{D}_{\mathfrak{V}, k+1}^{\dagger}$ and the general case follows from taking a finite presentation of it as $\mathcal{D}_{\mathfrak{V}, k+1}^{\dagger}$-module. However, $\left(\mathcal{K}_{k}(\mathfrak{V})\right)$ forms a $\left(\mathcal{D}_{k}^{\dagger}\right)$-sequence for the coadmissible module $\mathcal{K}(\mathfrak{V})$ and hence 3.1 .18 is bijective. So 3.1 .17 is bijective and $\mathcal{K} \in \mathcal{C}_{\mathfrak{X}}$. This implies $\lim _{k}^{(1)} \mathcal{K}_{k}=0$ by the preceding theorem and hence $\mathcal{J}=\lim _{k} \mathcal{J}_{k}$ where $\mathcal{J}_{k}=\operatorname{im}\left(f_{k}\right)$. Again, we have a linear map

$$
\mathcal{D}_{\mathfrak{X}, k}^{\dagger} \otimes_{\mathcal{D}_{\mathfrak{X}, k+1}^{\dagger}} \mathcal{J}_{k+1} \longrightarrow \mathcal{J}_{k}
$$

whose bijectivity follows with the same argument as above. Hence $\mathcal{J} \in \mathcal{C}_{\mathfrak{X}}$ and this suffices to conclude that the category $\mathcal{C}_{\mathfrak{X}}$ is abelian.

We conclude with a result on how the category $\mathcal{C}_{\mathfrak{X}}$ behaves under morphisms over $\mathfrak{X}_{0}$.

Proposition 3.1.19. Let $\mathfrak{X}^{\prime} \rightarrow \mathfrak{X}_{0}$ be another admissible formal blow-up, and let $\pi: \mathfrak{X}^{\prime} \rightarrow$ $\mathfrak{X}$ be a morphism over $\mathfrak{X}_{0}$. Then, for every coadmissible $\mathcal{D}_{\mathfrak{X}^{\prime}, \infty}$-module $\mathcal{M}$ the sheaf $\pi_{*} \mathcal{M}$ is a coadmissible $\mathcal{D}_{\mathfrak{X}, \infty}$-module via the isomorphism $\pi_{*} \mathcal{D}_{\mathfrak{X}^{\prime}, \infty}=\mathcal{D}_{\mathfrak{X}, \infty}$. Moreover, one has $R^{q} \pi_{*} \mathcal{M}=0$ for all $q>0$ and an equivalence of categories

$$
\pi_{*}: \mathcal{C}_{\mathfrak{X}^{\prime}} \stackrel{\simeq}{\longrightarrow} \mathcal{C}_{\mathfrak{X}} .
$$

Proof. Write $\mathcal{M}=\lim _{k} \mathcal{M}_{k}$ with coherent $\mathcal{D}_{\mathfrak{X}^{\prime}, k^{-}}^{\dagger}$ modules $\mathcal{M}_{k}$. By 2.3.8 we know that each sheaf $\pi_{*}\left(\mathcal{M}_{k}\right)$ is a coherent $\mathcal{D}_{\mathfrak{X}, k}^{\dagger}$-module. Moreover $\pi_{*}(\mathcal{M})=\lim _{k} \pi_{*}\left(\mathcal{M}_{k}\right)$, and

$$
\mathcal{D}_{\mathfrak{X}, k}^{\dagger} \otimes_{\mathcal{D}_{\mathfrak{X}, k+1}^{\dagger}}^{\dagger} \pi_{*} \mathcal{M}_{k+1}=\pi_{*} \mathcal{D}_{\mathfrak{X}^{\prime}, k}^{\dagger} \otimes_{\pi_{*} \mathcal{D}_{\mathfrak{X}^{\prime}, k+1}^{\dagger}} \pi_{*} \mathcal{M}_{k+1} \simeq \pi_{*}\left(\mathcal{D}_{\mathfrak{X}^{\prime}, k}^{\dagger} \otimes_{\mathcal{D}_{\mathfrak{X}^{\prime}, k+1}^{\dagger}} \mathcal{M}_{k+1}\right) \simeq \pi_{*} \mathcal{M}_{k}
$$

Note here that the exact functor $\pi_{*}$, cf. 2.3.8, indeed commutes with the tensor product: this may be checked over an open affine $\mathfrak{V} \subseteq \mathfrak{X}^{\prime}$ where we may take a finite presentation 
of the restriction of the module $\mathcal{M}_{k+1}$ to $\mathfrak{V}$ as $\mathcal{D}_{\mathfrak{V}, k+1}^{\dagger}$-module to reduce to the case of the sheaf $\mathcal{D}_{\mathfrak{V}, k+1}^{\dagger}$, as in the proof of the preceding theorem. This shows $\pi_{*} \mathcal{M} \in \mathcal{C}_{\mathfrak{X}}$. We now write $\mathcal{M}=\lim _{k} \mathcal{M}_{k}^{\prime}$ with coherent $\widehat{\mathcal{D}}_{\mathfrak{X}, k}^{(k, 0)}$-modules $\mathcal{M}_{k}^{\prime}$. Because of [25, 20.32.2] the functors $R \pi_{*}$ and $R \lim _{k}$ commute. By 3.1 .15 we have $\mathcal{M}=R \lim _{k} \mathcal{M}_{k}^{\prime}$. Hence we obtain $R \pi_{*}(\mathcal{M})=R \pi_{*}\left(R \varliminf_{k} \mathcal{M}_{k}^{\prime}\right) \simeq R \varliminf_{k} R \pi_{*}\left(\mathcal{M}_{k}^{\prime}\right)$. By 2.3.8 we have $R \pi_{*}\left(\mathcal{M}_{k}^{\prime}\right)=\pi_{*}\left(\mathcal{M}_{k}^{\prime}\right)$, and so we obtain a canonical isomorphism $R \pi_{*}(\mathcal{M}) \simeq R \varliminf_{k} \pi_{*}\left(\mathcal{M}_{k}^{\prime}\right)$. In the beginning of the proof we have seen that $\pi_{*} \mathcal{M}=\lim _{k} \pi_{*}\left(\mathcal{M}_{k}^{\prime}\right)$ is a coadmissible $\mathcal{D}_{\mathfrak{X}, \infty}$-module, and so $R \varliminf_{k} \pi_{*}\left(\mathcal{M} k_{k}^{\prime}\right)=\pi_{*}(\mathcal{M})$, by 3.1.15, From $R \pi_{*}(\mathcal{M})=\pi_{*}(\mathcal{M})$ we conclude that $R^{q} \pi_{*} \mathcal{M}=0$ for all $q>0$.

3.2. Coadmissible $\mathcal{D}$-modules on the Zariski-Riemann space $\left\langle\mathfrak{X}_{0}\right\rangle$. We finally explain how to pass the previous construction and results to the projective limit in $\mathfrak{X}$, that is to say, to the Zariski-Riemann space of $\mathfrak{X}_{0}$.

3.2.1. Let $\mathcal{F}_{\mathfrak{X}_{0}}$ be the set of all admissible formal blow-ups $\mathfrak{X} \rightarrow \mathfrak{X}_{0}$. 5 This set is partially ordered by setting $\mathfrak{X}^{\prime} \geq \mathfrak{X}$ if the blow-up morphism $\mathfrak{X}^{\prime} \rightarrow \mathfrak{X}_{0}$ factors as $\mathfrak{X}^{\prime} \stackrel{\pi}{\longrightarrow} \mathfrak{X} \rightarrow \mathfrak{X}_{0}$, where $\mathfrak{X} \rightarrow \mathfrak{X}_{0}$ is the blow-up morphism. The morphism $\pi: \mathfrak{X}^{\prime} \rightarrow \mathfrak{X}$ is then uniquely determined by the universal property of blowing up, and is itself a blow-up morphism [18, ch. 8, 1.24], and we will denote it henceforth by $\pi_{\mathfrak{X}^{\prime}, \mathfrak{X}}$. By [ 8 , Remark 10 in sec. 8.2] the set $\mathcal{F}_{\mathfrak{X}_{0}}$ is directed in the sense that any two elements have a common upper bound, and we can consider the topological space equal to the projective limit 6

$$
\left\langle\mathfrak{X}_{0}\right\rangle=\lim _{\mathfrak{X}_{\in} \in \mathcal{F}_{\mathfrak{X}_{0}}} \mathfrak{X}
$$

This is the Zariski-Riemann space associated with $\mathfrak{X}_{0}$. For its basic properties we refer to [8, 9.3].

3.2.2. Sheaves on the space $\left\langle\mathfrak{X}_{0}\right\rangle$. For $\mathfrak{X} \in \mathcal{F}_{\mathfrak{X}_{0}}$ we denote the canonical projection map $\left\langle\mathfrak{X}_{0}\right\rangle \rightarrow \mathfrak{X}$ by $\mathrm{sp}_{\mathfrak{X}}$. If $\mathfrak{X}^{\prime} \geq \mathfrak{X}$ in $\mathcal{F}_{\mathfrak{X}_{0}}$, we have $\mathrm{sp}_{\mathfrak{X}}=\pi_{\mathfrak{X}^{\prime}, \mathfrak{X}} \circ \mathrm{sp}_{\mathfrak{X}^{\prime}}$. The isomorphism $\left(\pi_{\mathfrak{X}^{\prime}, \mathfrak{X}}\right)_{*} \mathcal{D}_{\mathfrak{X}^{\prime}, \infty}=\mathcal{D}_{\mathfrak{X}, \infty}$ from 3.1 .6 , together with the adjunction map $\pi_{\mathfrak{X}^{\prime}, \mathfrak{X}}^{-1} \circ\left(\pi_{\mathfrak{X}^{\prime}, \mathfrak{X}}\right)_{*} \rightarrow$ id gives rise to a canonical map

$$
\varphi_{\mathfrak{X}, \mathfrak{X}^{\prime}}: \pi_{\mathfrak{X}^{\prime}, \mathfrak{X}}^{-1} \mathcal{D}_{\mathfrak{X}, \infty}=\pi_{\mathfrak{X}^{\prime}, \mathfrak{X}}^{-1}\left(\pi_{\mathfrak{X}^{\prime}, \mathfrak{X}}\right)_{*} \mathcal{D}_{\mathfrak{X}^{\prime}, \infty} \longrightarrow \mathcal{D}_{\mathfrak{X}^{\prime}, \infty} .
$$

These morphisms of sheaves satisfy

$$
\varphi_{\mathfrak{X}, \mathfrak{X}^{\prime \prime}}=\varphi_{\mathfrak{X}^{\prime}, \mathfrak{X}^{\prime \prime}} \circ \pi_{\mathfrak{X}^{\prime \prime}, \mathfrak{X}^{\prime}}^{-1} \varphi_{\mathfrak{X}, \mathfrak{X}^{\prime}}
$$

\footnotetext{
${ }^{5}$ We emphasize that the blow-up morphism $\mathfrak{X} \rightarrow \mathfrak{X}_{0}$ is part of the datum of $\mathfrak{X}$.

${ }^{6}$ In the paper [17] this space is denoted by $\mathfrak{X}_{\infty}$.
} 
whenever $\mathfrak{X}^{\prime \prime} \geq \mathfrak{X}^{\prime} \geq \mathfrak{X}$. We then obtain an inductive system $\left(\operatorname{sp}_{\mathfrak{X}}^{-1} \mathcal{D}_{\mathfrak{X}, \infty}\right)_{\mathfrak{X} \in \mathcal{F}_{\mathfrak{X}_{0}}}$ of sheaves of rings on $\left\langle\mathfrak{X}_{0}\right\rangle$, and we put

$$
\mathcal{D}_{\left\langle\mathfrak{X}_{0}\right\rangle}=\underset{\mathfrak{X}}{\lim } \operatorname{sp}_{\mathfrak{X}}^{-1} \mathcal{D}_{\mathfrak{X}, \infty}
$$

Definition 3.2.4. A $\mathcal{D}_{\left\langle\mathfrak{X}_{0}\right\rangle}$-module $\mathcal{M}$ is called coadmissible if there is a family $\left(\mathcal{M}_{\mathfrak{X}}, \psi_{\mathfrak{X}, \mathfrak{X}^{\prime}}\right)$ of coadmissible $\mathcal{D}_{\mathfrak{X}, \infty}$-modules $\mathcal{M}_{\mathfrak{X}}$, for all $\mathfrak{X} \in \mathcal{F}_{\mathfrak{X}_{0}}$, together with an isomorphism

$$
\psi_{\mathfrak{X}^{\prime}, \mathfrak{X}}^{\mathcal{M}}:\left(\pi_{\mathfrak{X}^{\prime}, \mathfrak{X}}\right)_{*} \mathcal{M}_{\mathfrak{X}^{\prime}} \stackrel{\simeq}{\longrightarrow} \mathcal{M}_{\mathfrak{X}},
$$

of $\mathcal{D}_{\mathfrak{X}, \infty}$-modules, whenever we have $\mathfrak{X}^{\prime} \geq \mathfrak{X}$ in $\mathcal{F}_{\mathfrak{X}_{0}}$. This system of modules and isomorphisms is required to satisfy the following conditions:

(i) Whenever $\mathfrak{X}^{\prime \prime} \geq \mathfrak{X}^{\prime} \geq \mathfrak{X}$ in $\mathcal{F}_{\mathfrak{X}_{0}}$ the following transitivity condition holds :

$$
\psi_{\mathfrak{X}^{\prime}, \mathfrak{X}}^{\mathcal{M}} \circ\left(\pi_{\mathfrak{X}^{\prime}, \mathfrak{X}}\right)_{*}\left(\psi_{\mathfrak{X}^{\prime \prime}, \mathfrak{X}^{\prime}}^{\mathcal{M}}\right)=\psi_{\mathfrak{X}^{\prime \prime}, \mathfrak{X}}^{\mathcal{M}} .
$$

(ii) $\mathcal{M}$ is isomorphic to the inductive limit $\underline{\lim }_{\mathfrak{X}} \mathrm{sp}_{\mathfrak{X}}^{-1} \mathcal{M}_{\mathfrak{X}}$ as $\mathcal{D}_{\left\langle\mathfrak{X}_{0}\right\rangle}$-module.

Note that the transition morphism $\mathrm{sp}_{\mathfrak{X}}^{-1} \mathcal{M}_{\mathfrak{X}} \rightarrow \mathrm{sp}_{\mathfrak{X}^{\prime}}^{-1} \mathcal{M}_{\mathfrak{X}^{\prime}}$ in the inductive limit in (ii) is defined by applying the functor $\mathrm{sp}_{\mathfrak{X}^{\prime}}^{-1}$ to the morphism

$$
\pi_{\mathfrak{X}^{\prime}, \mathfrak{X}}^{-1} \mathcal{M}_{\mathfrak{X}} \simeq \pi_{\mathfrak{X}^{\prime}, \mathfrak{X}}^{-1}\left(\pi_{\mathfrak{X}^{\prime}, \mathfrak{X}}\right)_{*} \mathcal{M}_{\mathfrak{X}^{\prime}} \rightarrow \mathcal{M}_{\mathfrak{X}^{\prime}}
$$

The latter is obtained from $\left(\psi_{\mathfrak{X}^{\prime}, \mathfrak{X}}^{\mathcal{M}}\right)^{-1}: \mathcal{M}_{\mathfrak{X}} \stackrel{\simeq}{\longrightarrow}\left(\pi_{\mathfrak{X}^{\prime}, \mathfrak{X}}\right)_{*} \mathcal{M}_{\mathfrak{X}^{\prime}}$ and the adjunction map $\pi_{\mathfrak{X}^{\prime}, \mathfrak{X}}^{-1} \circ\left(\pi_{\mathfrak{X}^{\prime}, \mathfrak{X}}\right)_{*} \rightarrow \mathrm{id}$.

We denote by

$$
\mathcal{C}_{\left\langle\mathfrak{X}_{0}\right\rangle} \subseteq \operatorname{Mod}\left(\mathcal{D}_{\left\langle\mathfrak{X}_{0}\right\rangle}\right)
$$

the full subcategory of coadmissible $\mathcal{D}_{\left\langle\mathfrak{x}_{0}\right\rangle}$-modules in the category of all $\mathcal{D}_{\left\langle\mathfrak{X}_{0}\right\rangle}$-modules.

Proposition 3.2.5. Let $\mathfrak{X} \in \mathcal{F}_{\mathfrak{X}_{0}}$. One has an equivalence of categories

$$
\left(\operatorname{sp}_{\mathfrak{X}}\right)_{*}: \mathcal{C}_{\left\langle\mathfrak{X}_{0}\right\rangle} \stackrel{\simeq}{\longrightarrow} \mathcal{C}_{\mathfrak{X}}
$$

In particular, the category $\mathcal{C}_{\left\langle\mathfrak{x}_{0}\right\rangle}$ is abelian.

Proof. Let $\mathcal{M}=\lim _{\mathfrak{X}} \mathrm{sp}_{\mathfrak{X}}^{-1} \mathcal{M}_{\mathfrak{X}}$ and let $\mathfrak{X}^{\prime} \geq \mathfrak{X}$. There is a canonical isomorphism

$$
\left(\operatorname{sp}_{\mathfrak{X}}\right)_{*} \operatorname{sp}_{\mathfrak{X}^{\prime}}^{-1} \mathcal{M}_{\mathfrak{X}^{\prime}} \stackrel{\simeq}{\longrightarrow} \mathcal{M}_{\mathfrak{X}} \text {. }
$$

Indeed, let $\mathfrak{U} \subseteq \mathfrak{X}$ be an open and let $\mathfrak{V}=\pi_{\mathfrak{X}^{\prime}, \mathfrak{X}}^{-1}(\mathfrak{U})$. Then

$$
\left(\operatorname{sp}_{\mathfrak{X}}\right)_{*} \operatorname{sp}_{\mathfrak{X}^{\prime}}^{-1} \mathcal{M}_{\mathfrak{X}^{\prime}}(\mathfrak{U})=\operatorname{sp}_{\mathfrak{X}^{\prime}}^{-1} \mathcal{M}_{\mathfrak{X}^{\prime}}\left(\operatorname{sp}_{\mathfrak{X}}^{-1}(\mathfrak{U})\right)=\mathcal{M}_{\mathfrak{X}^{\prime}}(\mathfrak{V})
$$

using that $\operatorname{sp}_{\mathfrak{X}^{\prime}}\left(\operatorname{sp}_{\mathfrak{X}}^{-1}(\mathfrak{U})\right)=\mathfrak{V}$. But $\mathcal{M}_{\mathfrak{X}^{\prime}}(\mathfrak{V}) \simeq \mathcal{M}_{\mathfrak{X}}(\mathfrak{U})$ via the map $\psi_{\mathfrak{X}^{\prime}, \mathfrak{X}}^{\mathcal{X}}$. 
In particular, we get an isomorphism

$$
\left(\operatorname{sp}_{\mathfrak{X}}\right)_{*}(\mathcal{M})=\underset{\mathfrak{X}^{\prime}}{\lim }\left(\operatorname{sp}_{\mathfrak{X}}\right)_{*} \operatorname{sp}_{\mathfrak{X}^{\prime}}^{-1} \mathcal{M}_{\mathfrak{X}^{\prime}} \stackrel{\simeq}{\longrightarrow} \mathcal{M}_{\mathfrak{X}} .
$$

This shows that the functor $\left(\mathrm{sp}_{\mathfrak{X}}\right)_{*}$ appearing in the proposition is well-defined. In the other direction, let $\mathcal{M} \in \mathcal{C}_{\mathfrak{X}}$ and define for $\mathfrak{X}^{\prime} \geq \mathfrak{X}$ the module $\mathcal{M}_{\mathfrak{X}^{\prime}}$ by the requirement $\left(\pi_{\mathfrak{X}^{\prime}, \mathfrak{X}}\right)_{*}\left(\mathcal{M}_{\mathfrak{X}^{\prime}}\right) \simeq \mathcal{M}_{\mathfrak{X}}$ via 3.1 .19 . The family $\left(\mathcal{M}_{\mathfrak{X}^{\prime}}\right)$ then satisfies the conditions (i) and (ii) in the above definition and its inductive limit $\mathcal{M}$ lies therefore in $\mathcal{C}_{\left\langle\mathfrak{x}_{0}\right\rangle}$. This gives a quasi-inverse to the functor $\left(\mathrm{sp}_{\mathfrak{X}}\right)_{*}$. By corollary 3.1 .16 , the category $\mathcal{C}_{\left\langle\mathfrak{X}_{0}\right\rangle}$ is then abelian.

Remark: It follows from the above proof that there is a canonical isomorphism of sheaves of rings

$$
\Gamma\left(\langle\mathfrak{X}\rangle, \mathcal{D}_{\left\langle\mathfrak{X}_{0}\right\rangle}\right) \simeq \Gamma\left(\mathfrak{X}, \mathcal{D}_{\mathfrak{X}, \infty}\right)
$$

for any $\mathfrak{X} \in \mathcal{F}_{\mathfrak{X}_{0}}$.

Theorem 3.2.6. (Theorem A and B for coadmissible $\mathcal{D}_{\left\langle\mathfrak{x}_{0}\right\rangle}$-modules)

(i) Let $\mathfrak{V} \subseteq \mathfrak{X}$ be an open affine and let $\langle\mathfrak{V}\rangle=\mathrm{sp}_{\mathfrak{X}}^{-1}(\mathfrak{V})$ be its Zariski-Riemann space. One has an equivalence of categories

$$
\Gamma(\langle\mathfrak{V}\rangle,-): \mathcal{C}_{\langle\mathfrak{V}\rangle} \stackrel{\simeq}{\longrightarrow} \mathcal{C}_{\mathcal{D}_{\mathfrak{V}, \infty}(\mathfrak{V})}
$$

(ii) For every $\mathcal{M} \in \mathcal{C}_{\left\langle\mathfrak{x}_{0}\right\rangle}$ and every $q>0$ one has

$$
H^{q}\left(\left\langle\mathfrak{X}_{0}\right\rangle, \mathcal{M}\right)=0 .
$$

Proof. Part (i) follows from the preceding proposition together with theorem 3.1.12, By [11, 0.3.1.19] the canonical map

$$
\underset{\mathfrak{X}}{\lim } H^{q}\left(\mathfrak{X}, \mathcal{M}_{\mathfrak{X}}\right) \stackrel{\simeq}{\longrightarrow} H^{q}\left(\left\langle\mathfrak{X}_{0}\right\rangle, \mathcal{M}\right)
$$

is an isomorphism. Thus, part (ii) follows from 3.1.15.

3.3. Examples. The first example is given by the structure sheaf of the Zariski-Riemann space tensored with $\mathbb{Q}$. Let us denote

$$
\mathcal{O}_{\left\langle\mathfrak{X}_{0}\right\rangle, \mathbb{Q}}=\underset{\mathfrak{X}}{\lim _{\mathfrak{X}}} \operatorname{sp}_{\mathfrak{X}}^{-1} \mathcal{O}_{\mathfrak{X}, \mathbb{Q}} .
$$

Proposition 3.3.1. The sheaf $\mathcal{O}_{\left\langle\mathfrak{x}_{0}\right\rangle, \mathbb{Q}}$ is a coadmissible $\mathcal{D}_{\left\langle\mathfrak{X}_{0}\right\rangle}$-module.

Proof. If $\mathfrak{X}, \mathfrak{X}^{\prime} \in \mathcal{F}_{\mathfrak{X}_{0}}$ and $\pi: \mathfrak{X}^{\prime} \rightarrow \mathfrak{X}$ is a morphism over $\mathfrak{X}_{0}$, then $\pi_{*} \mathcal{O}_{\mathfrak{X}^{\prime}, \mathbb{Q}}=\mathcal{O}_{\mathfrak{X}, \mathbb{Q}}$ by 2.3.1, By 3.2 .5 we have an equivalence of categories $\mathcal{C}_{\left\langle\mathfrak{X}_{0}\right\rangle} \rightarrow \mathcal{C}_{\mathfrak{X}_{0}}$ with an explicit quasi-inverse. From these considerations we see that the claim will follow from the fact that $\mathcal{O}_{\mathfrak{X}_{0}, \mathbb{Q}} \in \mathcal{C}_{\mathfrak{X}_{0}}$. 
For any integer $k$, the sheaf $\mathcal{O}_{\mathfrak{X}_{0}, \mathbb{Q}}$ is a $\mathcal{D}_{\mathfrak{X}_{0}, k}^{\dagger}$-module. Let $\mathfrak{U}_{0} \subset \mathfrak{X}_{0}$ an affine open of $\mathfrak{X}_{0}$ with coordinates $x_{1}, \ldots, x_{M}$, and corresponding derivations $\partial_{1}, \ldots, \partial_{M}$. Following 2.1.14, we write

$D_{k}^{\dagger}=\Gamma\left(\mathfrak{U}_{0}, \mathcal{D}_{\mathfrak{X}_{0}, k}^{\dagger}\right)=\left\{\sum_{\underline{\nu}} \varpi^{k|\underline{\nu}|} a_{\underline{\nu}} \underline{\partial} \underline{[\underline{\nu}} \mid a_{\underline{\nu}} \in \mathcal{O}_{\mathfrak{X}_{0}, \mathbb{Q}}\left(\mathfrak{U}_{0}\right)\right.$, and $\left.\exists C>0, \eta<1|| a_{\underline{\nu}} \mid<C \eta^{|\underline{\mid}|}\right\}$.

We have the following lemma, using the notation $\underline{0}=(0, \ldots, 0)$.

Lemma 3.3.2. Let $P \in D_{k}^{\dagger}$, there exist $P_{1}, \ldots, P_{M} \in D_{k}^{\dagger}$ and $a_{\underline{0}} \in \mathcal{O}_{\mathfrak{X}_{0}, \mathbb{Q}}\left(\mathfrak{U}_{0}\right)$ such that

$$
P=a_{\underline{0}}+\sum_{i=1}^{M} P_{i} \cdot \partial_{i}
$$

Proof. The proof of this lemma is essentially the proof of the Spencer lemma by Berthelot [5, 3.2.1] for the case $k=0$, meaning for the sheaf of arithmetic differential operators. Let us denote $\underline{1}=(1,0, \ldots, 0) \in \mathbb{N}^{M}$ and by $O P_{1}$ the set of operators in $D_{k}^{\dagger}$ such that $a_{\underline{\nu}}=0$ if $\nu_{1} \neq 0$. Let $P=\sum_{\underline{\nu}} \varpi^{k|\underline{\nu}|} a_{\underline{\nu}} \underline{\partial}^{[\underline{\nu}]} \in D_{k}^{\dagger}$, and consider

$$
P_{1}=\sum_{\underline{\nu} \mid \nu_{1} \neq 0} \varpi^{k|\underline{\nu}|} \underline{\underline{\underline{\nu}}}_{\nu_{1}} \underline{\partial}^{[\underline{\nu}-\underline{1}]}
$$

then, as $\left|1 / \nu_{1}\right|_{p}=O\left(\left|\nu_{1}\right|_{p}\right)=O\left(|\underline{\nu}|_{p}\right)$ when $|\underline{\nu}|_{p} \rightarrow+\infty$, this operator $P_{1}$ belongs to $D_{k}^{\dagger}$ (here $|\cdot|_{p}$ is the usual $p$-adic norm over the field $\mathbb{Q}$ ). Moreover since we have the identity

$$
\nu_{1} \underline{\partial}^{[\underline{\nu}]}=\partial_{1} \cdot \underline{\partial}^{[\underline{\nu}-\underline{1}]},
$$

we get that $P=P_{1} \partial_{1}+Q_{1}$ where $Q_{1} \in O P_{1}$. We can now apply the same procedure to $Q_{1}$ relatively to $\partial_{2}$. Doing this, we see that there exist $P_{2} \in D_{k}^{\dagger}$ and $Q_{2}$ with no terms containing neither $\partial_{1}$, nor $\partial_{2}$, such that $P=P_{1} \partial_{1}+P_{2} \partial_{2}+Q_{2}$. We finally find the lemma iterating $M$ times. Note that the $a_{0}$ term given by the lemma is necessarily the same as the initial $a_{\underline{0}}$ term of $P$.

This allows us to prove the

Lemma 3.3.3. There is a presentation

$$
\begin{gathered}
\mathcal{D}_{\mathfrak{U}_{0}, k}^{\dagger} \stackrel{\psi}{\longrightarrow} \mathcal{D}_{\mathfrak{U}_{0}, k}^{\dagger} \longrightarrow \mathcal{O}_{\mathfrak{U}_{0}, \mathbb{Q}} \longrightarrow 0 \\
\left(P_{1}, \ldots, P_{M}\right) \longrightarrow \sum_{i=1}^{M} P_{i} \partial_{i} . \\
P \longrightarrow P \cdot 1
\end{gathered}
$$

Proof. Let $\mathfrak{V}_{0} \subset \mathfrak{U}_{0}$ be affine, and denote by $D_{k}^{\dagger}=\Gamma\left(\mathfrak{V}_{0}, \mathcal{D}_{\mathfrak{X}_{0}, k}^{\dagger}\right)$, we have to prove that we have a presentation, with the same maps as in the statement

$$
D_{k}^{\dagger} \longrightarrow D_{k}^{\dagger} \longrightarrow \mathcal{O}_{\mathfrak{X}_{0}, \mathbb{Q}}\left(\mathfrak{V}_{0}\right) \longrightarrow 0 \text {. }
$$


Let $P \in D_{k}^{\dagger}, P=\sum_{\underline{\nu}} \varpi^{k \mid \underline{\nu}} a_{\underline{\nu}} \underline{\partial} \underline{\underline{\nu}]}$, such that $P(1)=a_{\underline{0}}=0$. By the previous lemma, there exist $P_{1}, \ldots, P_{M}$ such that $P=\sum_{i=1}^{M} P_{i} \partial_{i}$ so that $P \in \operatorname{im}(\psi)$.

Let us come back now to the proof of the proposition. Using this presentation, we see that $\mathcal{O}_{\mathfrak{X}_{0}, \mathbb{Q}}$ is a coherent $\mathcal{D}_{\mathfrak{X}_{0}, k}^{\dagger}$-module, and that we have canonical compatibility relations

$$
\mathcal{D}_{\mathfrak{X}_{0}, k}^{\dagger} \otimes_{\mathcal{D}_{\mathfrak{X}_{0}, k+1}^{\dagger}} \mathcal{O}_{\mathfrak{X}_{0}, \mathbb{Q}} \simeq \mathcal{O}_{\mathfrak{X}_{0}, \mathbb{Q}} .
$$

Finally the $\mathcal{D}_{\mathfrak{X}_{0}, \infty}$-module $\mathcal{O}_{\mathfrak{X}_{0}, \mathbb{Q}}$ is isomorphic to the constant projective system of coherent $\mathcal{D}_{\mathfrak{X}_{0}, k}^{\dagger}$-modules $\left(\mathcal{O}_{\mathfrak{X}_{0}, \mathbb{Q}}\right)$ and is an element of $\mathcal{C}_{\mathfrak{X}_{0}}$. As explained at the beginning of the proof, this implies that $\mathcal{O}_{\left\langle\mathfrak{x}_{0}\right\rangle, \mathbb{Q}} \in \mathcal{C}_{\left\langle\mathfrak{X}_{0}\right\rangle}$. This ends the proof of the proposition.

For the second example, we consider a Cartier divisor $\mathfrak{Z}$, which is assumed to be smooth over $\mathfrak{o}$, of the formal scheme $\mathfrak{X}_{0}$. As above, we denote by $\mathfrak{X}_{0, \mathbb{Q}}$ and $\mathfrak{Z}_{\mathbb{Q}}$ the rigid analytic spaces associated with $\mathfrak{X}_{0}$ and $\mathfrak{Z}$, respectively. Let $U=\mathfrak{X}_{0, \mathbb{Q}} \backslash \mathfrak{Z}_{\mathbb{Q}}$ be the open complement, and $j: U \rightarrow \mathfrak{X}_{0, \mathbb{Q}}$ the inclusion of rigid spaces. We have the specialization map sp : $\mathfrak{X}_{0, \mathbb{Q}} \rightarrow \mathfrak{X}_{0}$.

Proposition 3.3.4. The sheaf $\mathrm{sp}_{*} j_{*} \mathcal{O}_{U}$ is a coadmissible $\mathcal{D}_{\mathfrak{X}_{0}, \infty}$-module.

Proof. We freely use the notation and terminology of [5, 4.0.1]. Let us consider

$$
\left.V_{k}=\mathfrak{X}_{0, \mathbb{Q}} \backslash\right] \mathfrak{Z}\left[|\varpi|^{k}\right.
$$

and $\mathcal{V}_{s}\left(V_{k}\right)$ the set of strict neighborhoods of $V_{k}$. Note that $V_{k}$ is well defined since $\mathfrak{Z}$ is a Cartier divisor of $\mathfrak{X}_{0}$. If $\mathfrak{V}=\operatorname{Spf} A$ is an open affine subset of $\mathfrak{X}_{0}$, such that $\mathfrak{Z} \cap \mathfrak{V}=V\left(t_{1}\right)$, then

$$
V_{k} \bigcap \mathfrak{V}_{\mathbb{Q}}=\left\{\left.x \in \mathfrak{V}_{\mathbb{Q}}|| t_{1}(x)|\geqslant| \varpi\right|^{k}\right\}
$$

Note that

$$
U=\bigcup_{k} V_{k} \text { and } U=\bigcup_{k} \bigcup_{W \in \mathcal{V}_{s}\left(V_{k}\right)} W
$$

We introduce also

$$
\mathcal{E}_{k}^{\dagger}=\underset{W \in \mathcal{V}_{s}\left(V_{k}\right)}{\lim _{*}} \operatorname{sp}_{* *} j_{W} \mathcal{O}_{W}
$$

where $j_{W}: W \rightarrow \mathfrak{X}_{0, \mathbb{Q}}$ is the inclusion in $\mathfrak{X}_{0, \mathbb{Q}}$ of a strict neighborhood $W$ of $V_{k}$. We have an inclusion $V_{k} \subset V_{k+1}$ and $V_{k+1}$ is a strict neighborhood of $V_{k}$, so that $\mathcal{V}_{s}\left(V_{k+1}\right) \subset \mathcal{V}_{s}\left(V_{k}\right)$. As a consequence, for any $k$, there is a canonical morphism $\mathcal{E}_{k+1}^{\dagger} \rightarrow \mathcal{E}_{k}^{\dagger}$. Moreover, since $\mathrm{sp}_{*}$ commutes with projective limits, we have

$$
\operatorname{sp}_{*} j_{*} \mathcal{O}_{U_{L}}=\underset{k}{\lim } \mathcal{E}_{k}^{\dagger} \cdot
$$

The proposition will follow from the

Lemma 3.3.5. (i) The sheaf $\mathcal{E}_{k}^{\dagger}$ is a coherent $\mathcal{D}_{\mathfrak{X}_{0}, k}^{\dagger}$-module. 
(ii) The canonical map $\mathcal{E}_{k+1}^{\dagger} \rightarrow \mathcal{E}_{k}^{\dagger}$ induces a canonical isomorphism of coherent $\mathcal{D}_{\mathfrak{X}_{0}, k^{-}}^{\dagger}$ modules,

$$
\mathcal{D}_{\mathfrak{X}_{0}, k}^{\dagger} \otimes_{\mathcal{D}_{\mathfrak{X}_{0}, k+1}^{\dagger}} \mathcal{E}_{k+1}^{\dagger} \simeq \mathcal{E}_{k}^{\dagger}
$$

Proof. Let $W$ be admissible open in $\mathfrak{X}_{L}$. Then $W$ is the generic fiber of some Zariski open $\mathfrak{W}^{\prime}$ of $\mathfrak{X}^{\prime}$ where pr $: \mathfrak{X}^{\prime} \rightarrow \mathfrak{X}_{0}$ is an admissible blow-up of $\mathfrak{X}_{0}$. Denote by $j^{\prime}$ the inclusion : $\mathfrak{W}^{\prime} \hookrightarrow \mathfrak{X}^{\prime}$. Then $j_{*}^{\prime} \mathcal{O}_{\mathfrak{W}^{\prime}, \mathbb{Q}}$ is a $\mathcal{D}_{\mathfrak{X}^{\prime}, k^{\prime}}^{\dagger}$-module, for $k^{\prime} \geqslant k_{\mathfrak{X}^{\prime}}$, so that the sheaf $\operatorname{sp}_{*} j_{W *} \mathcal{O}_{W}=\operatorname{pr}_{*} j_{*}^{\prime} \mathcal{O}_{\mathfrak{W}^{\prime}, \mathbb{Q}}$ has an action of $\mathcal{D}_{\mathfrak{X}_{0}, k^{\prime}}^{\dagger}$ as $\mathcal{D}_{\mathfrak{X}_{0}, k^{\prime}}^{\dagger}=\operatorname{pr}_{*} \mathcal{D}_{\mathfrak{X}^{\prime}, k^{\prime}}^{\dagger}$ by 2.3.8. In particular, the sheaf $\operatorname{sp}_{*} j_{W *} \mathcal{O}_{W}$ is a $\mathcal{D}_{\mathfrak{X}_{0}, \infty}$-module for any admissible open $W$. As a consequence, the sheaf $\mathcal{E}_{k}^{\dagger}$ has a structure of $\mathcal{D}_{\mathfrak{X}_{0}, \infty}$-module as well. Let us check locally that this structure extends to a structure of $\mathcal{D}_{\mathfrak{X}_{0}, k}^{\dagger}$-module. Let $\mathfrak{V}=\operatorname{Spf} A \subset \mathfrak{X}_{0}$ be affine open in $\mathfrak{X}_{0}$ such that $\mathfrak{Z} \bigcap \mathfrak{V}=V\left(t_{1}\right)$ where $t_{1}$ is a local coordinate on $\mathfrak{V}$. Then we have the following description, where $A_{L}=A \otimes L$ is an affinoid algebra,

$$
\mathcal{E}_{k}^{\dagger}(\mathfrak{V})=\left\{\sum_{\nu \geqslant 0} a_{\nu} \varpi^{k \nu} t_{1}^{-\nu-1}, a_{\nu} \in A_{L}|\exists C>0, \eta<1|\left|a_{\nu}\right|<C \eta^{\nu}\right\} .
$$

Denote by $\partial_{1}$ the derivation corresponding to the coordinate $t_{1}$, and $\partial_{2}, \ldots, \partial_{M}$ the other derivations. Let us denote $D_{k}^{\dagger}=\mathcal{D}_{\mathfrak{X}_{0}, k}^{\dagger}(\mathfrak{V})$, then we have the following description using 2.1 .14

$$
D_{k}^{\dagger}=\left\{\sum_{\underline{\nu}} a_{\underline{\underline{\nu}}} \varpi^{k|\underline{\nu}|} \underline{\partial}^{[\underline{\nu}]} \mid a_{\underline{\underline{\nu}}} \in A_{L}, \text { and } \exists C>0, \eta<1|| a_{\underline{\underline{\nu}}} \mid<C \eta^{|\underline{\nu}|}\right\} .
$$

To prove the lemma it is thus enough to check the

Lemma 3.3.6. There is a presentation

$$
\begin{gathered}
D_{k}^{\dagger M} \longrightarrow D_{k}^{\dagger} \stackrel{\varphi}{\longrightarrow} \mathcal{E}_{k}^{\dagger}(\mathfrak{V}) \longrightarrow 0 \\
\left(P_{1}, \ldots, P_{M}\right) \longrightarrow P_{1} \partial_{1} t_{1}+\sum_{i=2}^{M} P_{i} \partial_{i} \\
P \longrightarrow P \cdot \frac{1}{t_{1}} .
\end{gathered}
$$

Proof. Again, we follow the proof by Berthelot [5, 4.4.2] of the analogous statement for arithmetic differential operators. It is clear that $\operatorname{im}(\psi) \subset \operatorname{ker}(\varphi)$. Observe that

$$
\partial_{1}^{\left[\nu_{1}\right]} \cdot t_{1}^{-1}=(-1)^{\nu_{1}} t_{1}^{-\nu_{1}-1} .
$$

Let $h=\sum a_{\nu} \varpi^{k \nu} t_{1}^{-\nu-1} \in \mathcal{E}_{k}^{\dagger}(\mathfrak{V})$, then $P=\sum_{\nu}(-1)^{\nu} \varpi^{k \nu} a_{\nu} \partial_{1}^{[\nu]}$ belongs to $D_{k}^{\dagger}$ and $P\left(1 / t_{1}\right)=$ $h$, so that $\varphi$ is surjective. Let now $P \in \operatorname{ker}(\varphi)$, then, applying repeatedly lemma 3.3.2, we see that modulo im $(\psi), P$ can be written $P=\sum_{\nu} \varpi^{k \nu} a_{\nu} \partial_{1}^{[\nu]}$, with coefficients $a_{\nu} \in A_{L}$ such that $\sum_{\nu} \varpi^{k \nu}(-1)^{\nu} a_{\nu}\left(t_{1}\right)^{-\nu-1}=0 \in \mathcal{E}_{k}^{\dagger}(\mathfrak{V})$. Moreover, there exist $C>0, \eta<1$ such 
that $\left|a_{\nu}\right|<C \eta^{\nu}$ where $|\cdot|$ is a Banach norm on $A_{L}$. Denote

$$
b_{j}=\sum_{\nu=0}^{j} a_{\nu} \varpi^{k \nu} t_{1}^{j-\nu}
$$

Let us now state the following

Auxiliary result 3.3.7. There exist $C^{\prime}>0, \eta^{\prime}<1$ such that $\left|b_{j}\right|<C^{\prime}|\varpi|^{k j} \eta^{\prime j}$.

Proof. Berthelot proved this lemma for $k=0$ in [5, 4.2.1]. Let us check that the proof can be adapted to any $k$. Since $\mathfrak{V}$ is smooth, the affinoid algebra $A_{L}$ is reduced, so that the spectral semi-norm is a norm and defines the Banach topology on $A_{L}$. All Banach norms being equivalent, we can use this norm to prove the statement, which we keep on denoting by $|\cdot|$. Let $\mathfrak{V}_{\mathbb{Q}}=\operatorname{Spf} A_{L}$ be the generic fiber of $\mathfrak{V}$, seen as rigid analytic space. Let $\eta^{\prime}>\eta$, such that $\eta^{\prime}<1$ and some power of $\eta^{\prime}$ lies in the valuation group of $L$. We consider the following admissible cover of $\mathfrak{V}_{\mathbb{Q}}$ by open $V_{1}$ and $V_{2}$ defined by

$$
V_{1}=\left\{\left.x \in \mathfrak{V}_{\mathbb{Q}}|| t_{1}(x)|\leqslant| \varpi\right|^{k} \eta^{\prime}\right\} \text { and } V_{2}=\left\{\left.x \in \mathfrak{V}_{\mathbb{Q}}|| \varpi\right|^{k} \eta^{\prime} \leqslant\left|t_{1}(x)\right| \leqslant 1\right\} .
$$

It is enough to bound the spectral norm of the $b_{j}$ on each of this affinoid open. As $A_{L}$ is reduced, $\Gamma\left(V_{1}, \mathcal{O}_{\mathfrak{X}_{L}}\right)$, resp. $\Gamma\left(V_{2}, \mathcal{O}_{\mathfrak{X}_{L}}\right)$, is reduced as well by Corollary 10 of [7, 7.3.2], so that the spectral norm induced a norm on these two affinoid open sets. If $x \in V_{1}$, then

$$
\left|b_{j}(x)\right|=\left|\sum_{\nu=0}^{j} \varpi^{k \nu} a_{\nu} t_{1}^{j-\nu}\right| \leqslant C|\varpi|^{k j} \eta^{\prime j} .
$$

Consider

$$
B=\left\{\sum_{\nu \geqslant 0} a_{\nu} \varpi^{k \nu} t_{1}^{-\nu-1}, a_{\nu} \in A_{L}|| a_{\nu} \mid \rightarrow 0\right\},
$$

which is the Banach algebra of analytic functions on the affinoid $\left\{\left.x \in \mathfrak{V}_{\mathbb{Q}}|| \varpi\right|^{k} \leqslant\left|t_{1}(x)\right| \leqslant\right.$ $1\}$. Obviously, $\mathcal{E}_{k}^{\dagger}(\mathfrak{V}) \subset B$. If $x \in V_{2}$, then

$$
\left|\varpi^{k \nu} a_{\nu} t_{1}^{-\nu}(x)\right| \leqslant C\left(\frac{\eta}{\eta^{\prime}}\right)^{\nu},
$$

so that the series $\sum_{\nu} \varpi^{k \nu}(-1)^{\nu} a_{\nu}\left(t_{1}\right)^{-\nu-1}$ converges to some element $b \in \Gamma\left(V_{2}, \mathcal{O}_{\mathfrak{X}_{L}}\right)$. Moreover the image of this element $b$ in $B$ is zero. The support of $b$ is a closed affinoid subset of $V_{2}$, and at each point $x$ of this support, $\left|t_{1}(x)\right|<\left|\varpi^{k}\right|$. By the maximum principle, increasing $\eta^{\prime}$ if necessary, provided that $\eta^{\prime}<1$, we can assume that the support is contained in $\left\{\left.x|| t_{1}(x)|\leqslant| \varpi\right|^{k}\left(2 \eta^{\prime}-1\right)\right\}$, so that $h=0$ restricted to $V_{2}$. Then we have the following upper bound for $x \in V_{2}$

$$
\left|b_{j}(x)\right|=\left|\sum_{\nu \geqslant j+1} \varpi^{k \nu} a_{\nu}\left(t_{1}(x)\right)^{j-\nu}\right| \leqslant C|\varpi|^{k j} \eta^{\prime j} .
$$


Let us come back to the proof of 3.3.6. We need to check that $P=\sum_{\nu} \varpi^{k \nu} a_{\nu} \partial_{1}^{[\nu]}$, such that $\sum_{\nu} \varpi^{k \nu}(-1)^{\nu} a_{\nu}\left(t_{1}\right)^{-\nu-1}=0 \in \mathcal{E}_{k}^{\dagger}(\mathfrak{V})$, belongs to $\operatorname{im}(\psi)$. Let us define

$$
b_{j}=(-1)^{j+1} \sum_{\nu=0}^{j-1}(-1)^{\nu} a_{\nu} \varpi^{k \nu} t_{1}^{j-\nu-1} \text {, }
$$

and

$$
Q=\sum_{j \geqslant 0} b_{j} \partial_{1}^{[j]}
$$

that belongs to $D_{k}^{\dagger}$ thanks to 3.3.7. Berthelot checked at the end of the proof of [5, 4.2.1], that $P=Q t_{1}$. But since this is true in $D_{0}^{\dagger}$, this is also true in $D_{k}^{\dagger}$ and $P=Q t_{1}$. By hypothesis, $b_{0}=0$, and by the lemma 3.3.2. this implies that there exists $Q_{1} \in D_{k}^{\dagger}$ such that $Q=Q_{1} \partial_{1}$. We finally conclude that $P=Q_{1} \partial_{1} t_{1}$ and that $P \in \operatorname{im}(\psi)$.

This presentation proves (i) and (ii) of the lemma 3.3.5 and completes the proof of 3.3.4.

\section{REFERENCES}

[1] K. Ardakov. $\widehat{\mathcal{D}}$-modules on rigid analytic spaces. Proceedings of the International Congress of Mathematicians 2014 Seoul,, III:1-9, 2014.

[2] K. Ardakov and S. Wadsley. $\mathcal{D}$-modules on rigid analytic spaces II: Kashiwara's equivalence. Journal of Algebraic Geometry, 27:647-701, 2018.

[3] K. Ardakov and S. Wadsley. $\mathcal{D}$-modules on rigid analytic spaces I. J. Reine u. Angew. Math., 747:221$276,2019$.

[4] Konstantin Ardakov and Simon Wadsley. On irreducible representations of compact $p$-adic analytic groups. Ann. of Math. (2), 178(2):453-557, 2013.

[5] P. Berthelot. Cohomologie rigide et théorie des $\mathcal{D}$-modules. In $p$-adic analysis (Trento, 1989), volume 1454 of Lecture Notes in Math., pages 80-124. Springer, Berlin, 1990.

[6] P. Berthelot. D-modules arithmétiques I. Opérateurs différentiels de niveau fini. Ann. Sci. E.N.S, 29:185-272, 1996.

[7] S. Bosch, U. Güntzer, and R. Remmert. Non-Archimedean analysis. Springer-Verlag, Berlin, 1984.

[8] Siegfried Bosch. Lectures on Formal and Rigid Geometry. Lecture Notes in Math., Vol. 2105. Springer-Verlag, Berlin, 2014.

[9] Bruno Chiarellotto and Bernard Le Stum. Pentes en cohomologie rigide et $F$-isocristaux unipotents. Manuscripta Math., 100(4):455-468, 1999.

[10] M. Emerton. Locally analytic vectors in representations of locally p-adic analytic groups. Preprint. To appear in: Memoirs of the AMS.

[11] K. Fujiwara and F. Kato. Foundations of Rigid Geometry I. Preprint, https://arxiv.org/abs/1308.4734.

[12] A. Grothendieck. Éléments de géométrie algébrique. IV. Étude locale des schémas et des morphismes de schémas IV. Inst. Hautes Études Sci. Publ. Math., (32):361, 1967.

[13] R. Hartshorne. On the De Rham cohomology of algebraic varieties. Inst. Hautes Études Sci. Publ. Math., (45):5-99, 1975.

[14] Robin Hartshorne. Residues and duality. Lecture notes of a seminar on the work of A. Grothendieck, given at Harvard 1963/64. With an appendix by P. Deligne. Lecture Notes in Mathematics, No. 20. Springer-Verlag, Berlin-New York, 1966. 
[15] Ryoshi Hotta, Kiyoshi Takeuchi, and Toshiyuki Tanisaki. D-modules, perverse sheaves, and representation theory, volume 236 of Progress in Mathematics. Birkhäuser Boston Inc., Boston, MA, 2008. Translated from the 1995 Japanese edition by Takeuchi.

[16] C. Huyghe. Dspł-affinité de l'espace projectif. Compositio Math., 108(3):277-318, 1997. With an appendix by P. Berthelot.

[17] C. Huyghe, D. Patel, T. Schmidt, and M. Strauch. $\mathcal{D}^{\dagger}$-affinity of formal models of flag varieties. Mathematical Research Letters (to appear).

[18] Qing Liu. Algebraic geometry and arithmetic curves, volume 6 of Oxford Graduate Texts in Mathematics. Oxford University Press, Oxford, 2002. Translated from the French by Reinie Erné, Oxford Science Publications.

[19] C. Noot-Huyghe. Finitude de la dimension homologique d'algèbres d'opérateurs différentiels faiblement complètes et à coefficients surconvergents. J. Algebra, 307(2):499-540, 2007.

[20] Christine Noot-Huyghe. Un théorème de Beilinson-Bernstein pour les D-modules arithmétiques. Bull. Soc. Math. France, 137(2):159-183, 2009.

[21] D. Patel, T. Schmidt, and M. Strauch. Integral models of $\mathbb{P}^{1}$ and analytic distribution algebras for GL(2). Münster J. Math., 7:241-271, 2014.

[22] D. Patel, T. Schmidt, and M. Strauch. Locally analytic representations of GL $(2, L)$ via semistable models of $\mathbb{P}^{1}$. Journal of the Institute of Mathematics of Jussieu, appeared online in January 2017.

[23] P. Schneider and J. Teitelbaum. Algebras of $p$-adic distributions and admissible representations. Invent. Math., 153(1):145-196, 2003.

[24] Atsushi Shiho. Notes on generalizations of local Ogus-Vologodsky correspondence. J. Math. Sci. Univ. Tokyo, 22(3):793-875, 2015.

[25] The Stacks Project Authors. Stacks Project. http://stacks.math.columbia.edu, 2017.

IRMa, Université de Strasbourg, 7 Rue René Descartes, 67084 Strasbourg cedex, France E-mail address: huyghe@math.unistra.fr

IRMar, Université de Rennes 1, Campus Beaulieu, 35042 Rennes cedex, France

E-mail address: Tobias.Schmidt@univ-rennes1.fr

Indiana University, Department of Mathematics, Rawles Hall, Bloomington, in 47405, U.S.A.

E-mail address: mstrauch@indiana.edu 